\title{
Abstracts from the \\ Center to Advance Palliative Care National Seminar Practical Tools for Making Change
}

\author{
October 26-29, 2016 \\ Orlando, Florida
}

The topic areas for abstract submission include:

- Measuring impact and value

- Palliative care education

- Specific patient populations

- Pediatrics

- Integration of palliative care to specific settings (e.g., hospital, ICU, home, nursing home, clinic)

- Staffing models

- Leveraging technology (e.g., EMR, telehealth)

- Equitable access to palliative care for vulnerable populations

- Advocacy and coalition building

- Health system strategies

- Payment models

- Care transitions

- Ensuring quality in advance care planning

- Team wellness 
1. Health Care Utilization Outcomes for LifeCourse Patients Allina Health

Minneapolis, MN

Team/Contact Information:

Jessica Taghon, BA

Research Associate

Allina Health

2925 Chicago Avenue, MR 10039

Minneapolis, MN 55407

(612) 262-1595

jessica.taghon@allina.com

Project Team Members:

Karl Fernstrom, $\mathrm{MPH}$

Heather Britt, $M P H, P h D$

Topic: Health system strategies

Description:

Health systems in the United States are faced with increased utilization for patients in their last years of life. Care for patients with serious illness is complex and requires a greater number of clinicians and care settings. This can contribute to duplicative and unwanted medical procedures. As a large portion of the population approaches retirement, health systems must redouble efforts to better serve patients as they near the end of life. LifeCourse is a patient-centered intervention, which leverages a layperson care guide to build upon an expanded set of palliative care domains. Care guides meet with patients, their family members and clinicians to help patients articulate goals, take part in decision making, and connect with resources. LifeCourse is a non-randomized prospective study of 450 intervention and 448 usual care patients followed between October 2012 and June 2016. Patients and controls were selected based on diagnosis, disease progression, and comorbidity mix. Using zeroinflated negative binomial regression models we tested whether participation in LifeCourse resulted in decreased utilization on three outcomes, ED visits, inpatient days, and ICU stays. On average, patients in the intervention group experienced $25 \%$ fewer inpatient days (IRR $=0.75 ; 95 \%$ CI: 0.62-0.91) and 57\% fewer ICU stays (IRR $=0.43 ; 95 \%$ CI: $0.24-0.77$ ). We did not detect a difference in the number of $E D$ visits (IRR $=0.85 ; 95 \%$ CI: 0.72-1.01). Our findings suggest that when a whole-person approach to care is used and patients' preferences are known there is a beneficial impact on health care utilization in late-life.

2. LifeCourse-Design to Implementation in a Health
System
Allina Health
Minneapolis, MN
Team/Contact Information:
Jessica Taghon, BA
Research Associate
Allina Health
2925 Chicago Avenue, MR 10039
Minneapolis, MN 55407
(612) 262-1595

jessica.taghon@allina.com

Project Team Members:

Paige Bingham, MBA

Vivian Anugwom, CHES

Scott Curran, MSW

Kendall Johnson, MHA

Sochenda Nelson

Laura Sutter, MPH

Topic: Health system strategies

\section{Description:}

In the next decade and a half, communities in the US will care for the same number of individuals over the age of 65 as they have over the past combined nine decades. The need for innovative, upstream palliative care models that can be deployed wide scale and sustainably is critical. A large healthcare delivery system in the upper Midwest has developed an innovative model-LifeCourse-to support patients facing advanced serious illness and their key friends and families.

Investigators began designing the model by conducting meetings with 183 patients, caregivers, and care professionals to systematically gather perspectives on optimal care delivery for persons diagnosed with serious illness. Participant feedback was shaped into care components that illustrate how illness is lived, the care relationship is experienced, and care is delivered. These care components guided the creation of protocols for a trained lay healthcare worker called a care guide who meets with patients and caregivers in their home early in their illness experience. Care guides work to understand the patient's experience and goals for living well, and help enhance supportive relationships in the family, community, and care teams.

LifeCourse is being evaluated through a research study that has enrolled 900 intervention and usual care patients and their key friends and family (total $n=1,800$ ). While data is still being collected on comparison patients, strong early results and supportive funding propelled leadership of the health system to implement LifeCourse across multiple clinics and collaborating community sites. This early deployment is part of an effort to close the widening gap between the production of positive research results and the systematic implementation of those interventions.

To successfully implement LifeCourse within existing care teams, an implementation team met with clinic leaders and key clinic staff over several weeks to help prepare the clinics, hire the care guides, and champion LifeCourse. LifeCourse worked this team to adapt program materials to suit the needs of their population, and review care guide protocols to make sure the clinic team understood the care guide work and how they could add value. In addition to following a detailed implementation strategy, an evaluation plan was created to measure the effectiveness and provide feedback on the process and outcomes. This poster will describe how LifeCourse moved from designing a new approach to late life care to systematically deploying this approach across a health system, and will summarize the successes and challenges of integrating research into practice.

\section{Improving QOL for Late Life Patients: LifeCourse} Findings

Allina Health

Minneapolis, MN

Team/Contact Information:

Jessica Taghon, BA

Research Associate

Allina Health

2925 Chicago Avenue, MR 10039

Minneapolis, MN 55407

(612) 262-1595

jessica.taghon@allina.com

Project Team Members:

Tetyana Shippee, $M S, P h D$

Nathan Shippee, MS, PhD

Karl Fernstrom, MPH

Patrick Mobley, MSAE 
Monica Frazer, MA, PhD

Heather Britt, $M P H, P h D$

Topic: Specific patient populations

\section{Description:}

Quality of life (QOL) for patients with chronic conditions at the end of life is a concern among policymakers and consumers, and has implications for improved care delivery outcomes. QOL indicators enable understanding of healthcare delivery beyond system oriented outcomes, shedding light on the effect of holistic, supportive care for those living with complex chronic illness in late life. In this mixed methods evaluation, we examine the effectiveness of a new patient-centered approach to late life care-LifeCourse intervention. A key component is ongoing, across-setting assistance by layperson care guides, supported by a clinical team. We investigate whether participation in LifeCourse improves QOL for patients, compared to usual care controls. QOL is assessed through quarterly administration of the Functional Assessment of Chronic Illness Therapy-Palliative Care survey ( $\mathrm{n}=190$ intervention patients and 157 controls). Data is presented as change in QOL indicators between baseline and 6 months. Mixed-methods analysis includes longitudinal examination of scores and qualitative data analysis. Multivariate analyses reveal that LifeCourse intervention had a significant positive effect on QOL for intervention patients when compared to controls. Additional analyses show that most changes happen for social quality of life domains. Interview data reveal that participants actively seek out ways to maintain QOL and exhibit resilience in the face of decline. Overall, our findings show that LifeCourse has a positive impact on QOL when compared to usual care patients. These trends indicate whole-person supportive care like LifeCourse is a promising approach for patients affected by complex chronic illness in late life.

\section{Speaking from the Heart: Heart Failure Goals beyond Advance Care Planning}

Allina Health, Division of Applied Research

Minneapolis, MN

Team/Contact Information:

Sandra E. Schellinger, NP

Nurse Practitioner, Senior Research Scientist

Allina Health, Division of Applied Research

2925 Chicago Avenue, So.

Minneapolis, MN 55407

(612) 262-1444

sandra.schellinger@allina.com

Project Team Members:

Monica Frazer, PhD

Karl M. Fernstrom, MPH

Scott M. Curran, MHP, LGSW

Topic: Ensuring quality in advance care planning

Description:

Honoring end-of-life wishes involves providing care in concordance with stated life sustaining treatment preferences usually specified in completed advance directives and advance care planning discussions. Heart failure is the most common admission diagnosis for older adults with high mortality and readmission rates and afflicts the whole person and burdens families over years. Knowing the goals and wishes of persons with serious illness is essential for individualizing care throughout the illness trajectory beyond care at the end of life.

This study aims to understand the evolution of medical and non-medical serious illness goals by asking advanced heart failure patients to describe what is most important to them. As part of LifeCourse, patients received monthly in-person visits by a lay health care worker, called a care guide. Care guides promoted whole-person, family-oriented care using assessment tools, whole-person and goals of care questions, and advance care planning discussions. Patients' self-defined goals and advance care plans were documented in the medical record. We examined the medical records of 30 heart failure patients who received supportive care greater than 347 days before death.

A qualitative analysis of medical record data-progress notes, advance care planning documentation, goals manager flowsheet-affirmed goals evolved over the illness experience. Goals that originally focused on physical (66\%) acquired psycho-social, emotional, and spiritual facets as patients came closer to end of life. Non-medical goals $(27 \%)$ occurred in the middle of the illness experience. An emotional or psychological element was often (80\%) attached to goals. Family/ caregiver goals $(26 \%)$ and aspirational goal statements $(13 \%)$ appeared consistently throughout the illness experience. Shifts in priorities along the illness trajectory identified specific turning points where proactive goals of care discussions could occur to realign care based upon the current health status and whole-person needs.

The dynamic nature of serious illness requires ongoing goals of care discussions that include the non-medical aspects of disease specific and life-sustaining treatments. Understanding what matters most and self-defined goals promote collaborative decision making that allows for individualized care and a whole person experience.

\section{State POLST Maturity and Advance Directive Completion}

Aspire Healthcare

Nashville, TN

Team/Contact Information:

Tara C. Friedman, MD, FAAHPM

Senior Medical Director

Aspire Healthcare

333 Commerce Street, Suite 700

Nashville, TN

(215) 375-7993

tfriedman@aspirehealthcare.com

Project Team Members:

Tara Friedman, MD, FAAHPM

Katy Lanz, DNP, MSN, AGPCNP-BC, ACHPN

Topic: Ensuring quality in advance care planning

Description:

Beyond advance directives, the presence of a completed POLST form assures a greater likelihood that an individual's preferences for medical treatment will be honored at the end of life. ${ }^{1,2}$ For more than 10 years, the National POLST Paradigm Task Force has assisted states in developing quality standards for POLST programs. To date, 19 states have endorsed POLST programs, 25 have developing programs and 3 states have programs that do not conform to requirements. ${ }^{3}$ Using a large multistate community-based palliative care program, we analyzed records from 71,768 nurse practitioner home visit encounters on 11,634 patients for presence of advance care planning conversation and completion of an advance directive (i.e., any state approved living will and/or POLST form). All care was provided by advance practice providers working for the same palliative care company in 1 of 4 states (two with endorsed POLST status (PA and TN), one with a developing POLST program (TX) and one whose program does not currently conform to POLST requirements $(\mathrm{AL})$. All staff received standardized training in 
communication and documentation of goals of care and advance directive completion. All patients received program education packets that included a copy of a state approved advance directive. In POLST program states, staff were also provided with a copy of the state's POLST form. Across all states, advance care planning was addressed at an average of $81.67 \%$ of advance practice provider home visits (range $66.99 \% \mathrm{TN}$ to $94.94 \% \mathrm{PA}$ ). Yet, documentation of patient treatment preferences was completed preferentially in the endorsed states over the developing state (TN 70\%, PA 72\% vs TX 39.9\%) and the non-conforming state (AL 26.8\%). This data demonstrates a correlation between the maturity of a state's POLST program and the completion of any advance directive document and support the efforts of the National POLST Paradigm Task Force.

\section{References}

1. Bomba PA, Kemp M. Black J. POLST: An improvement over traditional advance directives. Clev Clin J of Med 2012; 79.7:457-464.

2. Hickman SE, Nelson CA, Perrin NA, et al. A comparison of methods to communicate treatment preferences in nursing facilities: traditional practice vs the physician orders for life sustaining treatments program. JAGS 2010;58:1241-48.

3. http://www.polst.org/wp-content/uploads/2016/05/2016.05. 02-POLST-State-Status.pdf.

6. Integrating of a Palliative Care Goals of Care Conversation in the Acute Care of the Elderly Unit

Baystate Medical Center

Minneapolis, MN

Team/Contact Information:

Erin J. Salvador, MD

Palliative Care Program Innovator

Baystate Medical Center

759 Chestnut Street

Springfield, MA 01107

(413) 794-3245

erin.salvadormd@baystatehealth.org

Project Team Members:

Erin J. Salvador, $M D$

Richard Conroy, PA-C

Kasey Lackenbach, $R N$

Mihaela Stefan, $M D, P h D$

Sarah Rossmassler, NP, ACHPN

Maura Brennan, MD, FAAHPM, HMDC

Topic: Integration of palliative care to specific settings (e.g., hospital, ICU, home, nursing home, clinic)

\section{Description:}

Background: Palliative care (PC) interventions such as goals of care conversations (GOCC) enhance goal-concordant care, improve quality of life, decrease readmissions, and lower cost of care. A dedicated Acute Care for Elders (ACE) unit was launched on a general medical floor at Baystate Medical Center (BMC), a tertiary care center in Western MA. Currently, no formal GOCC are done within BMC by the primary team. A preliminary analysis on ACE readmissions found that roughly $30 \%$ of patients had unmet palliative care needs, and $40 \%$ of patients did not have a health care proxy (HCP) documented two days into admission. The aim of this quality improvement initiative was to assess the feasibility of screening for unmet PC needs and the impact of a GOCC in the ACE population.

Intervention: A multidisciplinary team including nursing, geriatrics, hospitalists, PC, and quality designed the intervention. Patients screened positive for the need of a GOCC if any of the following were present: 1) Provider answered yes to the Surprise Question (SQ), 2) $>=2$ admissions in the prior year, 3) Edmonton Frailty Score $>=12$. The Serious Illness Conversation Guide (SICG) was chosen as the standard GOCC format. A geriatric-trained physician assistant (GPA) performed and documented GOCC and communicated with relevant providers. MOLST forms were completed, as needed, and documented in the EHR, given to patients, and sent to providers.

Results: Among the 92 patients evaluated in two months, 49 $(53 \%)$ screened positive, and $20(41 \%)$ GOCC were completed (number limited by GPA time constraints). All GOCC were documented in the EHR and communicated to the hospital-, primary care- and post-acute providers. $18 / 20$ patients had a $\mathrm{HCP}$ in the chart before GOCC, and two were encouraged to bring their completed HCP to hospital. Before GOCC 5/20 patients had a MOLST in place. Ten patients had MOLST completed with DNR/DNI orders during the GOCC (five of which reflected a change from full code status). One significantly changed care plans, going home on Hospice with a "Do Not Return to Hospital" order. All remaining patients without MOLST were full code. DNR/DNI orders were documented on a MOLST for all patients who expressed this preference during the GOCC. Feedback from patients and families, nurses, and physicians was overwhelmingly positive.

Conclusions: Early experience suggests that this novel intervention of a GPA proactively performing GOCC in seriously ill elderly hospitalized patients is feasible. It may facilitate completion of documentation of patient's care preferences for life sustaining treatments across health care settings. The intervention is lower cost than a formal PC consult. We plan to collect data to clarify the impact of this intervention on readmissions, health care costs, and patient experience. This integration of PC and geriatrics is a potential new model of care for seriously ill hospitalized elderly that can extend scarce PC resources.

7. Immersive Palliative Care Education in a Virtual World Boston Medical Center/Tufts University School of Medicine Beverly, MA

Team/Contact Information:

Suzanne E. Mitchell, MD

Assistant Professor, Family Medicine and Palliative Care Boston Medical Center/Tufts University School of Medicine 4 Ives Street

Beverly, MA 01915

(617) 638-8000

suzanne.mitchell@bmc.org

Project Team Members:

Amy Lee, $M D$

Rebecca Koeniger-Donohue, DNP

Topic: Palliative care education

Description:

Significance: Barriers to effective end-of-life (EOL) care include the lack of health professional education around palliative care and advanced care planning (ACP), the absence of interprofessional education (IPE) in this field and the poor availability of palliative care services compared to the growing demand.

Methods: We conducted a pilot study to test the feasibility and acceptability of a new approach to IPE in palliative care using virtual world technology to convene students from different health professional training programs. Fifteen students from a Boston area medical school and partnering nursing and social work graduate program at a Boston area health professions 
college participated in a 2-hour workshop teaching communication around ACP using simulation scenarios and standardized patient actors in a virtual world environment. Participants engaged in the program remotely and following the training completed post-workshop surveys to provide feedback on their experiences.

Results: Confidence discussing ACP with patients and families increased for all participants after the workshop as compared to before participation, and many felt the combination of social interaction and anonymity the virtual world offers allowed for a judgment-free environment to engage in a topic that has been historically difficult for health professionals to discuss. $77 \%$ of respondents said they would definitely recommend this educational program. All but one respondent found navigating the technology very easy or manageable. Other participants appreciated the convenience of talking to people from other professions and ease of accessibility the virtual world affords. The majority of participants believed the workshop helped to remind them to "remain open, reflective, honest and empathetic," and to "slow down and ask more questions."

Conclusion: The virtual world ACP workshop positively influenced participants familiarity, knowledge and comfortlevel with ACP, supporting the use of virtual world technology as an engaging learning environment for health professionals to start dialogues around sensitive topics, and learn effective communication skills.

Summary: Our virtual world workshop proved feasible and effective in increasing students' familiarity and confidence discussing advance care planning.

\section{Advance Care Planning in Cancer: Using a Novel SOI Tool}

Boston Medical Center

Boston, MA

Team/Contact Information:

Sandhya Rao, MD

Director, Palliative Care Consult Service

Boston Medical Center

801 Massachusetts Avenue

Boston, MA 02118

(617) 638-8000

sandhya.rao@bmc.org

Project Team Members:

Alexandra Dobie, MSW, LCSW

Nicole Lincoln, $M S, R N, A P R N-B C, C C R N$

Diane Sarnacki, RN, MSN, AOCN

Gretchen Gignac, MD

Topic: Ensuring quality in advance care planning

Description:

Background: In cancer patients, advance directives are often not addressed until near the end of life. Medical care is often not aligned with patients' goals given inconsistent communication between health-care providers, patients, and caregivers. At Boston Medical Center (BMC), a 496-bed Academic Safety Net Medical Center, a 6-months retrospective readmissions study was performed in 2014 on the hematology-oncology inpatient population. Of this study population, $21 \%$ had a documented DNR order, $12 \%$ received a Palliative Care consult, and $17 \%$ had an end of life discussion, demonstrating that goals of care are not consistently addressed.

Patients with advanced disease and high symptom burden benefit from palliative care and Advance Care Planning (ACP). Twaddle et al. (2007) urges the development of systematic methods that identify seriously ill patients early in their admission to address goals of care and improve access to palliative care.

Objectives:

- To standardize triggers for palliative care and/or ACP discussions in patients with advanced disease who are on the hematology-oncology inpatient service at BMC

- To standardize ACP documentation in the Electronic Medical Record (EMR) at BMC to facilitate communication amongst providers regarding patient/family goals.

Methods: A novel Severity of Illness (SOI) tool was created. ACP discussions and/or palliative care consults were indicated within 72 hours for those who score 4 or greater on the tool. Hematology-oncology providers were educated about the intervention during their grand rounds. All patients admitted to the hematology-oncology service from January 4, 2016 through June 30, 2016 were scored during multi-disciplinary rounds. Retrospective chart reviews determined whether the proposed interventions were completed. BMC providers created a new ACP documentation template in the EMR.

Results: 352 patients admitted to the Hematology/Oncology inpatient service were scored using the SOI tool. $111(31.53 \%)$ of these patients received a score of 4 or greater; $53(47.74 \%)$ of this group received a palliative care consult and/or an $\mathrm{ACP}$ discussion.

Conclusions: The SOI tool promoted awareness among staff of the need for ACP discussions and/or palliative care involvement in identified patients. Although significantly increased from baseline, the tool alone did not translate into consistent application of these interventions. Barriers included inconsistent communication amongst providers. Only the senior resident was present at multi-disciplinary rounds and thus the attendings were not always informed about their patients' scores. Also, the ACP documentation template was inconsistently utilized, creating ambiguity regarding previously held ACP discussions. There is a need for education about ACP and exploration of beliefs about ACP among providers.

Implications: The data highlights the need to validate the SOI tool and formalize its use in the EMR. Further study, including interviews of providers, is needed to identify and address barriers and facilitators to standardizing triggers for ACP discussions and their documentation. This research has the potential to improve the quality of care cancer patients receive by consistent identification of patients that would benefit from ACP discussions and palliative care involvement.

\section{Venting Concerns: A Terminal Extubation Guideline} Butler Health System

Boston, MA

Team/Contact Information:

Lisa K. Doverspike, PA

Physician Assistant

Butler Health System

One Hospital Way

Butler, PA 16001

(724) 968-5330

lisa.doverspike@butlerhealthsystem.org

Project Team Members:

Lisa Doverspike, MPA PA-C

Matthew Schnur, PharmD

Margaret Kuhnlein, LCSW 
Dillon Stein, DO

Kathy Selvaggi, MS MD FAAHPM

Topic: Integration of palliative care to specific settings (e.g., hospital, ICU, home, nursing home, clinic)

Description:

The goal of withdrawing life-sustaining treatments is to discontinue therapies that are no longer desired or no longer providing comfort to patients. If not appropriately treated, patients who die after the withdrawal of mechanical ventilation can experience pain, dyspnea, and other distressing symptoms. Family members witnessing this may experience complicated grief after the patient's death. In August 2015, a Palliative Care program was initiated at Butler Health System (BHS). BHS, located 35 miles north of Pittsburgh, Pennsylvania, is a single hospital system anchored by Butler Memorial Hospital (BMH), a 297 bed community hospital located within a county of 185,000 residents. The Intensive Care Unit (ICU) within BMH is a 24 bed unit that averages 1100 admissions and 110 deaths per year $(10 \%)$. One of the initial priorities of the palliative care program was integration into the ICU. The ICU team was very interested in collaborating with the new palliative care program to improve care for critically ill patients.

A multidisciplinary task force of physicians, nurses, pharmacists, nurse practitioners, physician assistants and social workers was assembled with representation from critical care, ICU nursing, pharmacy, ethics and palliative care. A needs assessment questionnaire was developed and sent electronically to all health care providers in the ICU. Survey results revealed a concern for a lack of consistent approach to end of life care and a desire to improve skills for caring for patients at the end of life. Seventy-five percent of survey respondents ranked issues with communication as a top barrier to a smoother end of life transition for patients who die in the ICU. Respondents identified withdrawal of mechanical ventilation in the dying patient as a process that lacked specific guidelines at our institution and created a high level of angst for ICU health care providers.

Based on literature reviews and best practice procedures, a guideline was created that included a step wise process for communication surrounding withdrawal of mechanical ventilation in the dying patient. We developed a standard order set for medications commonly used in this process to control symptoms before and after extubating. This order set was reviewed and approved by the BMH Critical Care, Pharmacy and Therapeutics, and Forms committees. The order set was uploaded for use by all clinicians in the electronic order entry system (Meditech). Video education to all ICU health care providers was provided by representatives from pharmacy and palliative care. A laminated pocket sized guideline for reference was also made available. Palliative care consultation continues to be available to assist with end of life care when requested.

We are hopeful that with the use of the electronic order sets, video education, and pocket guides, a more consistent approach for withdrawal of mechanical ventilation will be implemented within the ICU. Our hypothesis is that staff satisfaction and confidence will increase with continued education and support. We will repeat the survey 6 months after implementation and continue ongoing education of newly hired ICU health care providers.

10. Bringing Palliative Care and Geriatrics to Primary Care Care New England Health System

Providence, RI

Team/Contact Information:

Ana Tuya Fulton, MD

Chief of Geriatrics

Care New England Health System
45 Willard Avenue

Providence, RI 02905

(401) 455-6362

afulton@carene.org

Project Team Member:

Kate M. Lally, MD

Topic: Integration of palliative care to specific settings (e.g., hospital, ICU, home, nursing home, clinic)

\section{Description:}

The Care New England Health System has spent the last few years on a journey to grow systems of care that focus on population health and better coordination of care and improved quality of care. Early in the journey, palliative care and geriatrics were identified as specialties to target for growth and integration. As part of the building of an accountable care organization (ACO), geriatrics and palliative care began efforts to outreach to the community to expand services outside the hospital walls. Community efforts included reaching out to the various affiliated primary care practices to recruit patients for a home based, nurse practitioner driven chronic care management program and to support primary care doctors in the care of complex, frail and often high utilizing older adults. We began to bring our teams (a geriatrician, a palliative care physician and a geriatric and palliative care trained nurse practitioner) to the primary care offices to reach out to the system's primary care doctors to identify high need patients for referral to palliative care or the ACO's home based team. With grant funding through the HRSA Geriatric Workforce Enhancement Program, this effort was expanded to include teaching and on-site case discussions to not only identify high need patients, but also to provide the primary care physician, their nurses and staff with education around topics of interest, having and encouraging goals of care conversations, and best practices in management of those with advanced illness. The efforts are well received and we hope to share this innovative way to bring geriatrics and palliative care into an integrated model into primary care to not only provide care to patients at need, but also to provide education and increased comfort to the staff and physicians practicing in primary care. We will share the model, several practice specific experiences and data around outcomes that are available at the time of the meeting.

\section{Scoring Suffering to Address Patient Needs}

Carle Hospital and Physician Group

Urbana, IL

Team/Contact Information:

Michael Aref, MD

Assistant Medical Director Medicine

Carle Hospital and Physician Group

611 W Park Street

Urbana, IL 61801

(217) 383-6744

michael.aref@carle.com

Project Team Members:

Michael Aref, MD, PhD, FACP, FHM

Catherine Simpson, MSW, LCSW

Emily Malecki, ACNP-BC

Barb Nation, RN, CHPN

Tim Staker, MDiv, BCC

Topic: Quality improvement

Description:

Introduction: Palliative care patients have been scored by their symptom burden and performance, but there is little standardization of their multidimensional suffering, needs, and wants. Maslow's Hierarchy of Needs is a model for describing 
these needs as physiological, safety, love/ belonging, esteem, and self-actualization. The functional pain score is a validated method of scoring pain based on patient report and provider assessment. Using these two frameworks, the "Maslow Score" seeks to score the current patient situation based on symptom burden, plan, network, and meaning.

Methods: The scores are four-digit codes describing the patient situation at a given time based on team consensus. Each digit is a score from most secure, 0 , to most vulnerable, 5 . Both written examples and an algorithmic approach have been provided to obtain each score.

Results: Morning huddle has been expedited by utilizing the "Maslow Scores" recorded the previous day. Changes in the clinical situation can be re-scored and compared against a team standard to improve both hand-off and follow-up needs. This triaging helps direct team resources as to whether patients should be reassessed by the entire team or specific members. The utilization of this scoring system has improved discussion and assessment of our patients from an interdisciplinary perspective.

Discussion: The "Maslow Score" appears to have improved the quality of care that our service delivers by improving efficiency. Further development and study is needed to standardize and validate our method.

\section{Preparing the Next Generation: Creation of a Palliative Care Fellowship}

Carolinas Healthcare System

Charlotte, NC

Team/Contact Information:

Rachel Snyder, APRN, BC

Carolinas Healthcare System

1000 Blythe Blvd.

Charlotte, NC 28203

(704) 355-2000

rachel.snyder@carolinashealthcare.org

Project Team Member:

Terri Long, MSN, ACNP-BC, ACHPN

Topic: Palliative care education

Description:

Objective: To describe the creation of a post graduate training program in palliative care that provides novice NP's a diverse clinical and didactic experience so they can transition into confident and competent providers.

Introduction: There is a national shortage of hospice and palliative-medicine physicians and demand will likely grow as the number of patients with life-limiting illness or chronic conditions increases. The current capacity of medical fellowship programs is insufficient to fill the shortage. Global demand is also rising according to the World Health Organization and Worldwide Palliative Care Alliance. Currently there is 1 palliative care physician for every 1,300 people diagnosed with a life threatening illness and 1 for every 20,000 patients with severe chronic illness. The shortage of Palliative Medicine physicians estimated at 6,000-18,000 in Dec 2010, and expected to increase. There are fewer than 300 fellowship trained MD's each year and approximately 5,000 current board certified MD's and many of these only work part time in Palliative Care. Currently there are fewer than 10 active nurse practitioner Palliative Care fellowship programs available in the US. Nurse practitioners could ideally help fill these positions in a much needed specialty area.

Description: In an attempt to meet the demands of this Palliative Care provider shortage, Carolinas Health Care Sys- tems (CHS) has developed a 12-month fellowship program for NP's and PA's. The Palliative Care Fellowship is designed to equip NP/PA's with the specialized skill sets necessary to assist patients and families who are diagnosed with a prolonged or life-threatening illness. With a focus on the provider's transition to practice, the program is structured to develop and strengthen clinical proficiencies while increasing competence and confidence alongside a multidisciplinary team of well-rounded healthcare providers. They have weekly didactic sessions supplemented by an online classroom. Completion of this exceptional fellowship affords graduates the expertise to deliver comprehensive healthcare services together with the care coordination services which are essential to support best quality of life and appropriate symptom management. They receive clinical training in hospice and palliative care as well as optional rotations with pain management, rural experience, leadership/ management, pediatrics and the post - acute care environment.

Data: The first cohort applicant pool included 8 applicants. Two candidates were selected after an interview process. These two applicants will graduate the Fellowship end of September 2016, with one Fellow staying within our Palliative group and the other returning to her home state. Our second cohort consisted of 4 applicants, one of which was selected after the interview process. They will complete the Fellowship end of March 2017.

Conclusion: This comprehensive post-graduate approach offers the advantage of a guided learning experience and direct patient care while in a protected learning environment which increases confidence and in turn presents a smoother transition to practice. With this structured, supported Fellowship early in the NP/PA practice, the hope is also greater retention within the hospital system.

13. Allowing Natural Death in the Emergency Department Cedars Sinai Emergency Department

Los Angeles, CA

Team/Contact Information:

Anthony Loffredo, MD

Emergency Physician, ED Director of Palliative Care

Cedars Sinai Emergency Department

8700 Beverly Blvd.

Los Angeles, CA 90048

(310) 423-3277

anthony.loffredo@cshs.org

Project Team Members:

Steven Shen

Anthony Loffredo, MD

Steven Rudd, MD

Joel Geiderman, MD

Sam Torbati, $M D$

Topic: Quality improvement

Description:

Study Objective: A $50 \%$ decrease in the mortality rate of Emergency Department (ED) patients nationwide was reported by Kanzaria in July, 2016. This is thought to be due, at least in part, to the increased proportion of deaths now occurring in hospice settings. Despite these trends, the demands of caring for a patient at the EOL, especially in the final hours, often motivates a visit to the ED. Emergency Physicians (EPs), who may be uncomfortable managing dying patients, are then charged with the complicated task of managing a critically ill patient and family who are typically emotionally overwhelmed and often conflicted. We propose a metric for this difficult situation: 
allowing natural death in the ED. In light of efforts to improve EOL care in our ED, and to increase Advance Care Planning (ACP) documentation in the surrounding community, we sought to quantify an improvement of this metric over the past 5 years.

Methods: This retrospective chart review was conducted at an academic urban Level 1 Trauma Center ED with an annual census of approximately 85,000 patients. Electronic medical records (EMR) of consecutive patients who expired in the ED in the years of 2011 and 2015 were reviewed. Patients who suffered cardiac arrest prior to ED arrival and could not be resuscitated were excluded from this analysis. All other patients who expired in the ED during these time periods were included for review regardless of age and gender. The reviewers determined whether natural death was allowed in the ED and what data points were used to assist with that decision, such as family request or preexisting ACP document, such as a Physician Orders for Life Sustaining Treatment (POLST), Do Not Resuscitate (DNR) orders from referring facilities, or Advanced Directives. Fisher's exact test was used for analyzing a potential difference between these two 12-month periods.

Results: ED deaths totaled 115 in 2011 and 113 in 2015, with natural death accounting for $19(16.5 \%)$ patients in 2011 compared to $35(30.9 \%)$ in $2015(\mathrm{p}<0.02)$. Three patients were terminally extubated in 2011 compared with 9 in 2015. Family input was the most common source of information leading to a decision towards natural death. Overall ED census, patient demographics, acuity, and ICU and non-ICU admission rates were similar in 2011 compared to 2015.

Discussion: The number of natural deaths allowed in our ED significantly increased in 2015 compared to 2011 suggesting increased EP comfort and expertise in appropriately managing patients at the end of life. Other contributing factors may include improved ACP documentation, patient and family education, or changes in ED culture. Further study may clarify the frequency of ACP documentation, missed opportunities for natural death in the ED, perhaps the additional longer term benefits of skillful EOL conversations by EP's, and opportunities to improve the quality of the EOL experience for patient and their families.

14. Team Wellness Strategies for Palliative Care Programs Cedars-Sinai Medical Center - Supportive Care Medicine Los Angeles, CA

Team/Contact Information:

Anna Lotakov, MHA

Program Manager

Cedars-Sinai Medical Center

Supportive Care Medicine

8700 Beverly Blvd, Becker 224

Los Angeles, CA 90048

(310) 423-2857

anna.lotakov@cshs.org

Project Team Members:

Anna Lotakov, MHA

Neha Darrah, MD

Azadeh Dashti, MD

Topic: Team wellness

Description:

Background: In the past two years, the Cedars-Sinai Supportive Care Medicine (SCM) team has experienced rapid increase in staffing as well as an unforeseen high turnover rate. It was evident that there was a need to assess the team's morale, wellness, and structure. As part of the FY16 goals, the team agreed to create a SCM Team Wellness Strategic Plan to address self-care and team structure which would ultimately contribute to the program's sustainability and growth.

Methods: With the support from administrative leadership, the SCM program held a $2 \frac{1}{2}$ day retreat that included all members of the SCM team, key leadership and staff from Cedars Sinai Medical Center, and nationally recognized palliative care consultants. The purpose of the retreat was to focus on improving the team's overall health and service standards. Through group discussions, team building activities, and practical skill building exercises, the team developed four unique and easily implementable strategies for promoting team health.

Results: The four wellness strategies developed during the retreat are detailed below.

- "Code lavender" process for intense cases - a code that can be called to alert the team when an individual is in personal/emotional/spiritual/existential/mental distress during the day.

- Self-care goals and plan for each member of the team Led by team chaplain, each team member defined their plan for self-care in the following areas: emotional, mental, physical, social and/or spiritual. The team also created a "buddy system" to support and encourage self-care. In order to keep each other on track, the team completes selfassessment ratings and identifies stressors during their daily huddles. Also during the monthly reflection sessions, the chaplain checks in with each team member about their self-care plan.

- Streamline of consult triaging - The triage RN is solely responsible for triaging all consults. Each morning the team discusses their schedule, meetings, and capacity for new patients. Also, the triage RN and other team members proactively communicate with referring providers to set service expectations.

- Recruitment for additional social work and administrative days - As our team continues to grow, the need for additional social work support is critical. Recognizing this need, SCM leadership budgeted for a third social worker for FY17. In addition to recruitment, administrative days were provided for the social workers.

Conclusion: The interventions implemented by the team resulted in quick, easy and effective changes that reduced the risk of compassion fatigue, promoted better self-care, and provided program sustainability. Future goals have been set to continue exploring and applying other interventions.

15. Alternative Payment for Palliative Care across Settings Center to Advance Palliative Care

New York, NY

Team/Contact Information:

Allison Silvers, MBA

Vice President, Payment and Policy

Center to Advance Palliative Care

55 W 125th Street, Suite 1302

New York, NY 10027

(212) 824-9572

allison.silvers@mssm.edu

Project Team Members:

Saskia Siderow

Allison Silvers, MBA

Topic: Integration of palliative care to specific settings (e.g., hospital, ICU, home, nursing home, clinic) 


\section{Description:}

The future of healthcare payment is here, presenting an unprecedented opportunity for palliative care. Alternative payment models (APMs) take many forms - shared savings, bundled payments, capitation and value-based purchasing - but they have one thing in common: they all increase provider responsibility for managing the quality of care and controlling unnecessary utilization. Palliative care has a strong track record on both quality and cost. By providing expert pain and symptom management, psychosocial support and skilled communication with patients and families, palliative care helps patients and families break the exhausting cycle of emergency room visits, hospital admissions, and unnecessary procedures. Not only does this reduce spending, but it vastly improves the patient experience.

We conducted exploratory research to learn how palliative care programs are integrating into models of alternative payment across the continuum of care; and to collect best practices and lessons learned from program leaders. Informational interviews were conducted with more than 20 program leaders around the United States. Programs cared for seriously ill patients across multiple settings and using multiple delivery models. They also had varying degrees of integration in alternative payment models, from the planning stages to fully integrated and at risk.

16. PC's Opportunity in Today's Changing Payment
Landscape
Center to Advance Palliative Care
New York, NY
Team/Contact Information:
Sara Mullery
Center to Advance Palliative Care
55 W 125th Street, Suite 1302
New York, NY 10027
(212) $201-2683$

sara.mullery@mssm.edu

Project Team Members:

Sara Mullery

Stacie Sinclair, MPP

Allison Silvers, MBA

Topic: Payment models

Description:

Introduction: The methods that Medicare, Medicaid and private payers use to reimburse providers for care are rapidly changing toward value-based purchasing (VBP) arrangements. Most VBP arrangements include some form of quality and cost tracking and reporting, linking performance on quality measures and resource use directly to provider payment.

Methods: Our research developed a comprehensive picture of current and proposed quality measurement impacting payment for physicians, medical homes, hospitals, nursing homes, home health agencies, accountable care organizations, cancer centers, and dialysis centers, as well as commercial and Medicare Advantage plans. From these, we identified the measures that palliative care has been shown to improve, based on literature review.

Findings: Palliative care positively impacts several crosscutting measures used to evaluate health plans and providers participating in VBP arrangements. Some of the measures that are used consistently across many of the VBP arrangements are hospital re-admissions, emergency department visits, and depression screening and management, all of which have been shown to improve when palliative care is delivered when compared to usual care.
In addition to these measures, we found another opportunity for palliative care to impact Medicare Advantage Plans and providers: the Consumer Assessment of Healthcare Providers and Systems (CAHPS) survey. The CAHPS survey measures patient experience of care via surveys provided to patients who received care in hospitals, accountable care organizations, patient-centered medical homes, cancer centers, dialysis centers, home health agencies, and nursing homes, and is also used to evaluate patients' experience with their health plan. Questions on CAHPS surveys include topics such as communication with doctors, pain management, and shared decision-makingall of which palliative care providers score highly on.

Conclusions: There are numerous opportunities for palliative care to improve an organization's performance scores. Their ubiquitous presence makes a strong argument for the integration of palliative care teams in all the settings, and suggests that palliative care integration can be financially beneficial as well. Additional research comparing financial performance under VBP arrangements for entities with palliative care integration to entities without is needed to confirm the potential benefits that we have found.

\section{Using Patient Data to Electronically Identify PC Patients Cerner Corporation \\ Kansas City, MO}

Team/Contact Information:

Heidi Gruhler, $\mathrm{MPH}$

Cerner Corporation

10234 Marion Park Drive

Kansas City, MO 64137

(816) 945-3922

heidi.gruhler@cerner.com

Project Team Members:

Heidi Gruhler, MPH - Cerner Corporation

Hannah Luetke-Stahlman, MPA - Cerner Corporation

April Krutka, DO - Intermountain Healthcare

Emmie Gardner, MSW - Intermountain Healthcare

Topic: Leveraging technology (e.g., EMR, telehealth)

Description:

Background: Palliative care (PC) has been shown to improve the quality of life of patients and their families facing the problems associated with chronic and serious illness through early identification, assessment, and prevention and treatment of pain and other problems, physical, psychosocial and spiritual. Patients with chronic and serious illness frequently move through the healthcare system in many different venues, such as hospitals, clinics, rehab facilities, and emergency rooms. There is growing evidence that timely integration of palliative care is needed in the acute care and inpatient hospital settings. Intermountain Healthcare, in collaboration with Cerner Corporation, developed a hospital-based electronic palliative care identification algorithm, which leverages historical and near real time data to identify patients that would benefit from secondary level palliative care services at the time of admission, along with an integrated clinical workflow solution to help providers decrease cost and improve quality of care.

Methods: This evaluation utilizes a non-randomized retrospective study design. A mixed-methods approach using qualitative and quantitative methods will help to triangulate the data and will offer a more in-depth look at palliative care solutions. We analyzed 30 days of clinical data from the Intermountain Healthcare system for patients who received palliative care consults, as well as for patients identified by the electronic 
algorithm. Key informant interviews were conducted after presenting the 30-day results to the palliative care team.

Results: The algorithm triggered 2,995 times on 1,384 unique patients, which was $70 \%$ of the total inpatient population during the 30 days. During this same time period, only 62 patients actually received palliative care consults, encompassing $3 \%$ of the total inpatient population. Qualifying criteria inclusive of diagnosis, hospital utilization, functional status and symptoms were reviewed. Interviews with the Intermountain palliative care teams provided the context and clinical expertise needed to understand what changes should be made to the algorithm to increase the accuracy of triggers. After making many of the proposed changes, we reanalyzed the same 30-day data. The number of times the algorithm triggered dropped to 992 on 425 unique patients, which was $21 \%$ of the total inpatient population.

Conclusion: A mixed-methods collaboration is successful for developing and optimizing a palliative care identification algorithm. We will re-iterate the same process in August/September, continuing our collaboration to refine the algorithm and have additional data results to present at CAPC's National Seminar in October.

\section{Impact of an Inpatient Palliative Care Program Christiana Care health System Newark, DE \\ Team/Contact Information: \\ John S. Emberger \\ Performance Improvement Program Manager \\ Christiana Care Health System \\ 4755 Ogletown-Stanton Road \\ Newark, DE 19713 \\ (302) 733-1000 \\ jemberger@christianacare.org \\ Project Team Members: \\ Roshni Guerry \\ Vinay Maheshwari \\ John Emberger \\ Anne Mueller \\ Topic: Measuring impact and value \\ Description:}

Background: Our Inpatient Palliative Care Team has been available for consult on patients with serious illness with the goal of: relieving the symptoms/stress of that serious illness, improving the quality of life and clarifying/aligning our Goals of Care with the patients desires for the management of that serious illness. While Inpatient Palliative Care can help the patient during the current admission, it was not well known if an inpatient consult can have benefits even after the patient is discharged. The goal of this project is to investigate if an Inpatient Palliative Care Team can reduce healthcare utilization after the patient is discharged from the hospital.

Methods: This project retrospectively reviewed all patients who received inpatient palliative care at Christiana Care from 4/ 1/15 to 6/30/15. Data collected included: Palliative Care chart documentation, Goals of Care documentation and information from our data warehouse concerning admissions and Emergency Department (ED) visits both 6 months prior to and 6 months after the admission that the patient received inpatient palliative care. We verified with the Delaware office of vital statistics that the included patients did not die during the 6 month post palliative care period.

Results: 309 patients were identified that were admitted between 4/1/15 and 6/30/15 who were consulted for Inpatient Palliative Care during that admission and had not died 6 months

\begin{tabular}{lrrc}
\hline Data displayed as: & PRE & POST & \%REDUCTION \\
\hline Inpatient Admissions: & 431 & 228 & $47 \%$ \\
Inpatient Days: & 2832 & 1497 & $47 \%$ \\
ICU Days: & 246 & 152 & $38 \%$ \\
ED Visits: & 155 & 128 & $17 \%$ \\
\hline
\end{tabular}

following the admission. During the 309 patients admission Palliative Care Service visited the patients a total of 822 times (2.7 per patient) and conducted 234 family meetings.

Data below shows the hospital and ED visits for this population 6 months PRE and 6 months POST the inpatient admission with palliative care.

Conclusion: While an outpatient Palliative Care program is the optimal way to reduce acute healthcare resources, an inpatient Palliative Care program can reduce hospital, ICU and ED visits.

\section{Piloting the Care Management and Palliative Care Program}

Christiana Care Health System

Newark, DE

Team/Contact Information:

Linsey D. O'Donnell, DO

Faculty, Department of Family and Community Medicine

Christiana Care Health System

4755 Ogletown Stanton Road

Newark, DE 19718

(302) 320-5700

lodonnell@christianacare.org

Project Team Members:

Linsey D. O'Donnell, DO

Kristen Isaac

Ina $\mathrm{Li}$

Topic: Integration of palliative care to specific settings (e.g., hospital, ICU, home, nursing home, clinic)

\section{Description:}

The goal of the Care Management and Palliative Care Program (CMAP) is to improve quality of life, and provide dignity and respect for homebound patients. Interventions include: intensive care management, incorporation of palliative medicine principles into care, utilization of patient centered care strategies for decreasing hospital utilization (therefore increasing time at home). Equally important is a focus on the patient oriented outcomes of increased symptom assessment, goals of care discussions, and completion of advance directives.

We identified a population within our home visit practice which is high risk for readmission and death. Eligibility: $\geq 60$ years old, a hospitalization or ED visit, approval from the patient's primary team of providers, scoring $\geq 5$ on the risk scoring tool applied after hospital/ED visit. The scoring tool is currently being validated and is based on internal data of homebound patients with high utilization and includes: utilization of hospital or ED (numeric), number of chronic conditions (CHF, COPD, ESRD, CVA, bedbound status, stage 3 or 4 pressure ulcers, AIDS, and advanced cancer), dual-eligible status, length of stay, hospital readmissions (numeric).

Analysis six month pre and post intervention $(n=51)$ has shown a significant decrease in hospital or ED visits from an average of $59 \mathrm{ED}$ visits pre-CMAP program to $32 \mathrm{ED}$ visits post-CMAP and from 142 inpatient visits pre-CMAP program to 51 visits post-CMAP program. Length of stay has been reduced from 16 days to 3.3 days on average pre-post intervention. 
Number of 30 day readmissions in the cohort decrease from 21 to 13. Patients are staying out of the hospital for an average of 84 days post intervention. One-hundred percent of patients have had discussions about completing advanced directives and $89 \%$ of patients have them scanned into the chart. One-hundred percent of patients have a documented symptom assessment.

The CMAP program demonstrates that using population health and palliative care principles improves outcomes for patients, paving the way for future outpatient palliative care initiatives in a home-based setting. The program demonstrates decrease in utilization of hospital/ED visits, 30-day readmission, and hospital length of stay. Results also demonstrate a more consistent approach to applying palliative interventions such as goals of care discussions and symptom assessment, which were completed in $100 \%$ of patients.

Next steps include validating the risk scoring/trigger tool, comparing the CMAP group to a historical cohort, dissemination of findings, and applying for future funding opportunities to continue this work.

Ultimately the CMAP team has been able to tailor care to the individual's needs with the goal of improving quality of life and respecting patients' health care choices, while improving health care system utilization.

20. Outpatient Palliative Cardiology Embedded within a Heart Failure Clinic: A New Model of Care

Cleveland Clinic

Cleveland, $\mathrm{OH}$

Team/Contact Information:

Krista R. Dobbie, MD

Staff Physician

Cleveland Clinic

9500 Euclid Avenue

Cleveland, $\mathrm{OH} 44106$

(216) 636-9770

dobbiek@ccf.org

Project Team Members:

Benjamin Gandesbery

Krista Dobbie, MD

Eiran Z. Gordeski

Topic: Integration of palliative care to specific settings (e.g., hospital, ICU, home, nursing home, clinic)

Description:

Introduction/Background: The role of specialist palliative care in the management of heart failure (HF) has been established in the contexts of in-patient HF care and management of patients with left ventricular assist devices (LVAD). However, the role of palliative care consultation in the outpatient treatment of HF patients is less well studied. In August 2015, we created an outpatient palliative care service embedded within the HF clinic at Cleveland Clinic Main Campus. Patients were referred by cardiologists based only on perceived palliative need; no triggers were used for patient referral.

Objectives: Our objectives were to characterize patients cared for by our novel outpatient palliative cardiology service, including their degree of HF, overall symptoms, comorbidities, palliative treatments, advanced directives status, and mortality.

Methods: We conducted a retrospective chart review of all patients evaluated by this outpatient service. Symptom burden was assessed with a modified Edmonton Symptom Assessment System (ESAS). Functional status was assessed with the Palliative Prognostic Index (PPI). Left ventricular ejection fraction (EF), HF stage, and NYHA class were recorded. Survival rate was calculated with the Kaplan-Meier method.
Results: Seventy-one patients were seen in 160 out-patient encounters. Twenty had an LVAD, three were heart transplant recipients, and 48 had symptomatic HF without advanced interventions. Most patients had NYHA class III symptoms $(68 \%)$ and were ACC/AHA stage $\mathrm{C}(61 \%)$, indicating symptomatic but not end-stage disease. Sixty-five percent had a PPI less than 2, $23 \%$ had a PPI between 2 and 4, and 13\% had a PPI greater than 4. Twenty-four percent of patients had coincident cancer diagnoses. Across all visits, the most commonly reported ESAS symptoms were tiredness $(74 \%)$, pain $(73 \%)$, dyspnea $(60 \%)$, depression (51\%), and anxiety (47\%). Across all patients, the most common palliative medications prescribed were opiates (59\%), antidepressants (44\%), laxatives (42\%), antineuropathics $(38 \%)$, and anxiolytics (32\%). Of patients reporting pain, $52 \%$ had muscular or skeletal pain, $45 \%$ had neuropathic pain, and $17 \%$ had medical device pain. Seventy percent had Advanced Directives in place. Mean survival was 576 days (SE 50 days).

Conclusions: We report our experience with a palliative cardiology service embedded within a HF clinic. Patients presented throughout the HF disease course with an array of quality-of-life limiting symptoms, and were medically managed with several interventions familiar to palliative medicine specialists.

Implications: Novel integrated models of cardiac-palliative outpatient care are feasible. Outpatients living with chronic HF have a significant symptom burden and may benefit from outpatient specialist palliative care, though prospective investigation of outcomes is needed.

\author{
21. Palliative Care Clerkship Pilot \\ Cleveland Clinic \\ Cleveland, $\mathrm{OH}$ \\ Team/Contact Information: \\ Laura K. Shoemaker, DO \\ Staff Physician, Interim Program Director \\ Cleveland Clinic \\ 9500 Euclid Avenue, T3 \\ Cleveland, $\mathrm{OH} 44106$ \\ (216) 444-5193 \\ shoemal@ccf.org \\ Project Team Members: \\ Laura K. Shoemaker, DO, MS \\ Mellar Davis, MD, FAAHPM \\ Kathleen Neuendorf, MD \\ Laura Hoeksema, MD \\ Topic: Palliative care education \\ Description: \\ Objectives:
}

1. Appreciate the role Palliative Care (PC) Clerkships play in undergraduate medical education.

2. Describe a multifaceted PC clerkship.

Background: The presence of formal, clinical PC training in US medical schools is variable, ranging from non-existent to weeks-long clerkships. Current approaches to PC teaching are inadequate, primarily preclinical, poorly integrated, focused on knowledge rather than skills and attitudes, and lack opportunity for reflection (Billings, 1997). A survey of Clerkship Directors found $75.8 \%$ of respondents felt that PC curricula should occur in clerkships, but only $43.6 \%$ had formal curricula (Shaheen, 2014). Early clinical experiences with critical illness, death, and dying have implications on development of students' professional identity, attitudes, and behaviors (Ratanawongsa, 2005). 
Prior to this Pilot, Cleveland Clinic's Lerner College of Medicine (CCLCM) did not have formal, clinical PC curriculum.

Objectives: Design a PC Clerkship wherein medical students: 1. Develop enhanced palliative care knowledge with basic competency in symptom assessment and management, relationship-centered communication, and care of the dying patient, 2. Understand and appreciate the role of an interdisciplinary team in health care delivery, 3. Develop a heightened level of empathy and self-awareness during medical practice.

Methods: Medical students participate in a PC experience consisting of 1. Direct patient care in four PC settings (Acute PC Unit, Inpatient Consultation, Outpatient Clinic, and Hospice), 2. Six Didactic Seminars 3. Reflective Writing.

Results: Nine third year CCLCM students completed the Pilot during the 2015-2016 academic year. Students completed a post clerkship assessment composed of: 1. Competence SelfAssessment, 2. Knowledge assessment (vonGunten, 2012), and 3. The Jefferson Scale of Physician Empathy, Medical Student version (Fields, 2011). Students submitted a Reflective Writing assignment in response to the prompt: "Please write about an experience you had with a terminally ill patient during this Palliative Care Clerkship. You may also consider the impact of caring for seriously ill and dying patients on your professional development as a physician." Quantitative and qualitative evaluation of these assessments is underway. Informal feedback from students and medical school administration has been overwhelmingly positive. Based on the first year's experience, the Dean has requested that time dedicated to PC curriculum be increased for the 2016-2017 academic year. Medical students are requesting PC electives with increased frequency.

Conclusions: The PC Clerkship Pilot is a multifaceted educational experience that has been well received by medical students and faculty.

Implications: Expanding opportunities for early PC clinical education should be a priority for medical educators. Programmatic assessment should be completed so the impact of these experiences can be defined and shared.

\section{References}

1. Billings J. JAMA 1997;289:733-738.

2. Shaheen A. Academic Medicine 2014;89:1168-1173.

3. Ratanawongsa N. Academic Medicine 2005;80:641-647.

4. von Gunten C. Journal of Palliative Medicine 2012;15:11981217.

5. Fields S. Journal of Interprofessional Care 2011;25:287-293.

\section{Advancing Compassionate, Patient/Family-Centered} Care

Atlanta VA Medical Center \& Emory University SOM

Atlanta, GA

Team/Contact Information:

Lynn Bunch O'Neill, MD

Associate Professor \& Associate Director, Emory Palliative Care Center

Atlanta VA Medical Center \& Emory University SOM

1185 The By Way

Atlanta, GA 30306

(919) 699-0880

lynn.oneill@emory.edu

Project Team Members:

Pamela Bowman, BSN, RN, OCN

Deborah Allen, PhD, RN, CNS, FNP-BC, AOCNP

Topic: Palliative care education

\section{Description:}

Background and Problem Statement: Upon development of a collaborative practice model (CPM) to provide best practices for the delivery of bad news (DBN), oncology nurses reported feeling inadequately prepared to provide support that patients and families need when receiving bad news. This staff education program supported the patient during and after DBN, an essential component of a CPM.

Goals and Objectives: Provide staff communication skills for supporting patients during DBN.

Increase staff satisfaction and comfort in discussions when providing support to patients after DBN.

Setting: 54 staff members working on 31-bed adult medicaloncology unit participated and included nurses, occupational and physical therapists, dietitians, social workers, and discharge planners.

Model or Program Description: A 3-hour education program developed through collaboration between a palliative care physician and nursing staff. Participants were provided communication skills to assist with DBN and responding to emotion. Patient and physician actors demonstrated a bad news conversation scenario. Participants learned how to be "present" for conversations by observing cognitive and affective elements of scenario. Using simulated skills practice, participants engaged in follow-up conversations with patient. Time-outs allowed for discussion with facilitator and peers. Participants were given opportunities to try skills again.

Evaluation: Using pre-post education surveys modeled after work culture surveys, participants reported increases in physician-nurse collaboration and their ability to support patients during DBN $(\mathrm{p}<0.05)$.

Discussion: Simulated skills practice was a new modality for educating our nurses. While the education program was primarily designed for nursing staff, interdisciplinary team inclusion was requested because of their interactions with patients in the wake of DBN. Initial grant funding provided actors and paid participant time away from work. Although funds for this level of support are no longer available, this successful program has been requested across the health system. Thus, training staff educators to facilitate and "act the parts" are the biggest barriers to broader implementation.

23. Does Changing the Name, Influence Patients' Code Status Choice?: Changing DNR (Do Not Resuscitate) to AND (Allow Natural Death)

Fresno Medical Education and Research/UCSF

Fresno, CA

Team/Contact Information:

Shane A. Lieberman, MD

Fresno Medical Education and Research/UCSF

155 N Fresno Street

Fresno, CA 93701

(559) 499-6400

SLieberman@fresno.ucsf.edu

Project Team Members:

Shane A. Lieberman, MD

John Miller, MD

Topic: Quality improvement

Description:

Context: Per the Patient Self Determination Act of 1990, upon admission to the hospital, patients are provided written notice of their decision-making rights, and policies regarding advance health care directives. As a result, all patients when 
being admitted to the hospital are asked about their code status choice. There is scant evidence that suggests changing the terminology "Do Not Resuscitate" (DNR) to "Allow Natural Death" (AND) could have an impact on a patient's code status choice in a theoretical situation. There is no evidence, however, that changing the language will affect a patient's code status choice upon admission.

Objectives: To determine if using standardized language including the phrase AND during the admission process will affect patient choice of code status.

Methods: Chi-squared test was used to compare code status of patients meeting criteria (age $>70$ or those of any age with a chronic disease or cancer) admitted to the family medicine inpatient service in Westminster, CO (metro-Denver area) using a standardized script incorporating AND to those admitted with normal, standard of care admission language, DNR. Intervention took place for 6 weeks, with historical control group from preceding 7 months.

Results: In the intervention group $(n=77) 37.7 \%$ chose AND. In the control group $(n=304) 21.7 \%$ chose DNR. Using a standardized script including "Allow Natural Death" made a statistically significant difference $(p=0.004)$ in code status choice among all patients.

Conclusion: Using the phrase "Allow Natural Death" makes a difference when discussing code status with patients.

\section{The "Opioid Square": A New Way to Learn Opioid Conversions \\ Georgetown University Hospital \\ Washington, DC \\ Team/Contact Information: \\ Heidi Young, MD \\ Assistant Professor \\ Georgetown University Hospital \\ 3800 Reservoir Road NW, 6PHC \\ Washington, DC 20007 \\ (855) 633-0364 \\ heidi.m.young@gunet.georgetown.edu \\ Project Team Members: \\ Heidi Young, MD \\ James Shear \\ Yvonne Hernandez \\ Peggy Compton}

Topic: Palliative care education

Description:

Objectives: We evaluated the "Opioid Square" in comparison to a classic conversion table in a single $2^{\text {nd }}$ year medical school class, assessing both accuracy in making opioid conversions and preference for each tool.

1. Determine the degree to which the "Opioid Square" tool assists 2nd year medical students to correctly calculate opioid conversions

2. Evaluate 2nd year medical student's preference to use the "Opioid Square" in comparison to the classic equianalgesic tables to calculate opioid conversions.

Background: Equianalgesic conversions between opioid drugs and oral and intravenous formulations are a clinically important skill for all practitioners who treat pain. Accuracy is critical to preventing serious adverse events. Opioid conversion tables are a commonly used tool for teaching this skill, however needs assessment at our institution reveals the classic conversion table is difficult for learners to understand. A novel visual tool, the "Opioid Square" was developed to provide a different visual framework for opioid conversions.
Methods: The sample consisted of 200 students at a single academic institution who were learning opioid conversions for the first time during their Pharmacology class. Students were taught conversion using both the standard conversion table, and the "Opioid Square" method. An eight item conversion quiz followed, on various opioid drugs and formulations of each. Students were allowed to use either tool and preferences were assessed with qualitative comments.

Results: 187/200 students participated in the study. Accuracy of the eight conversion questions was generally good (85-100\% correct), and without any statistical differences in accuracy between the Square and the Table. Incorrect conversions were more likely on two-step problems (i.e.: IV fentanyl to oral morphine). A slight majority of students (53\%) indicated that they preferred the Opioid Square over the Conversion Table, and $35 \%$ noted they would prefer to use both tools if given the option in the future.

Conclusions: The novel "Opioid Square" tool is not inferior to the classic opioid equianalgesic table for helping students make accurate conversions. As many students preferred the "Square," this tool may provide educators with an alternative visual framework for teaching opioid conversions. Our poster will provide education on using this tool at any institution, and pocket cards will be provided.

A link to the "Opioid Square" tool is:

https://www.dropbox.com/s/gdabbj9t144293v/OpioidSquare .pdf?dl=0

\section{PCforMe: Preparing Patients for Palliative Care}

Global Palliative Care Quality Alliance

Durham, NC

Team/Contact Information:

Jonathan Nicolla, MBA

Clinical Trials Coordinator, Duke University

Global Palliative Care Quality Alliance

2424 Erwin Road

Durham, NC 27705

(919) 668-4907

jonathan.nicolla@duke.edu

Project Team Members:

Jonathan Nicolla, MBA

Tisha Broyles

Debra Davis

Fred Friedman, $B A$

Laura Roe

Arif Kamal, MD, MBA, MHS

Topic: Palliative care education

\section{Description:}

There are many misconceptions held by patients with chronic or life-limiting disease regarding the role of palliative care as a part of their treatment plan. For example, many patients equate palliative care to hospice or end-of-life care. Due to this misconception, there is a high cancellation rate of new palliative care consults. To provide patients and caregivers better information regarding palliative care and how it differs than hospice or end-oflife care, better tools are needed for patients and caregivers.

To date, current interventions for patients to learn about palliative care are limited and unreliable. Typically, to learn how palliative care can be implemented into a treatment regimen, a patient must 1) speak directly to his/her doctor which many patients are hesitant to do, 2) discuss with friends or family who have experienced palliative care in the past, or 3) search for information on the internet which can often be misleading or unreliable. 
To address this issue, we have created PCForMe, a webbased interactive tool that helps educate and prepare patients for their initial palliative care consult. PCforMe empowers patients to become more engaged in making decisions regarding their care. Utilizing a mobile technology platform, PCforMe allows palliative care patients to learn about palliative care at their own pace. Using videos and interactive gaming concepts, PCforMe helps prepare palliative care patients for their initial consult with a palliative care clinician. Throughout PCforMe, patients will be prompted to submit information about what is most important to them, what they need help with, their hopes and worries about the future, and what they need to prepare for their palliative care journey. Once patients complete their PCforMe experience, the application automatically compiles all responses into a document called the Palliative Care Passport. The Palliative Care Passport is a printable document that allows patients to share their responses with their caregivers, loved ones, or clinical team.

We will continue to make iterations of the content and capabilities of PCforMe as we test it with patients, caregivers, doctors, and other stakeholder groups. First, we will develop usability and feasibility protocols to test PCforMe in a clinical setting at the Duke Cancer Institute. Revisions of this website will occur based on the feedback of this testing.

\section{Prepped: Guiding Patients toward Productive Visits with Their Physician \\ Duke University \\ Durham, NC}

Team/Contact Information:

Fred Friedman, BA

Clinical Research Coordinator, Duke University

2424 Erwin Road

Durham, NC 27705

(919) 668-1998

fred.friedman@duke.edu

Project Team Members:

Fred Friedman, $B A$

Jonathan Nicolla, MBA

Nicholas Wysham, MD

Arif Kamal, MD

Topic: Leveraging technology (e.g., EMR, telehealth)

Description:

Patients who receive a diagnosis of serious illness may find themselves with a significant amount of stress. While the internet is a great source of information, it may neither be correct, nor be pertinent to the specifics of their condition. Often, the most valuable source of information is the patient's visit with their provider. However, if the patient does not know what questions they should be asking, the visit may not be as fruitful as it could be.

A team of researchers at Duke University has created an app called Prepped to help bridge this gap. Prepped allows patients (or caregivers) to find questions that are best suited to the patient, questions that have been pre-selected by clinicians, and questions that are used most by other patients with the same illness. The patient is then able to create a list of questions that correspond with each visit to see their physician.

Currently, Prepped is focused on two disease types: cancer and COPD. Prepped's cancer-centered version establishes the patient's cancer type (breast, lung, prostate, etc.), their perceived stage along their cancer journey (diagnosis, pretreatment, survivorship, etc.), their gender, and their age range. Prepped's COPD-centered version asks similar demographic questions, along with questions specific to COPD symptom burden, such as shortness of breath.

Using this patient-entered information, as well as tags on each question, Prepped is able to match a unique patient with the most relevant questions for him or her. For example, a 28year-old female patient with breast cancer may see questions about breastfeeding, whereas a 70 -year-old male patient with prostate cancer would not. This level of personalization will help a patient zero in on the best questions for them.

Prepped was designed with the intent to include more disease types. The structure of the app would support any type of disease and would simply require the introductory questions to be written. This is the future of the app.

\section{Utilization of Lean Six Sigma Methodology Palliative Care}

Gulfside Hospice \& Pasco Palliative Care, Inc.

Land O' Lakes, FL

Team/Contact Information:

Kathleen P. Postiglione, MBA, BSN, CHC

Chief Operations Officer

Gulfside Hospice \& Pasco Palliative Care, Inc.

2061 Collier Parkway

Land O' Lakes, FL 34639

(727) 484-7934

Kathy.postiglione@ghppc.org

Project Team Members:

Kathleen Postiglione, MBA, BSN, CHC

Charles L Suggs, MD

Topic: Measuring impact and value

Description:

Utilizing Lean Six Sigma methodology, we were able to determine the financial value of the "feeder" benefit of the Palliative Care program to the Hospice Care business division. Specifically, to identify the \% palliative care conversions (palliative care consults that convert to hospice admissions) required to "break even" or reduce palliative care business unit deficit.

28. Teaching End of Life at the Beginning of the Journey Hofstra Northwell School of Medicine

Hempstead, NY

Team/Contact Information:

Brian E. Emmert, Jr., BS

Hofstra Northwell School of Medicine

500 Hofstra Boulevard

Hempstead, NY 11549

(516) 242-7478

bemmer1@pride.hofstra.edu

Project Team Members:

Brian Emmert, $B S$

Danielle Qing, $B A$

Tara Liberman, DO

Topic: Palliative care education

Description:

Introduction: With recent strides in medicine, a larger proportion of older adults are living longer with chronic disease. As a consequence, greater numbers of people will experience suffering, especially during the advanced stages of illness. Palliative and hospice medicine play an integral role in the care and well-being of this demographic of patients. One potential area of significant improvement in the delivery of palliative and hospice care is early education of future doctors in the intricacies and 
importance of these subspecialties during medical school. However, topics such as end-of-life-care can be sensitive and there is concern regarding the most appropriate time to introduce palliative and hospice care within a medical school curriculum. More research is needed to elucidate students' readiness to learn these crucial subspecialties along their education.

Methods: A voluntary, uncompensated, 13-item survey was administered to all eligible students at Hofstra Northwell School of Medicine via e-mail blast. The survey canvassed students' knowledge about hospice/palliative care and opinion regarding the teaching of these subspecialties during the first two years of medical school.

Results: Students over all four years had a strong interest in learning hospice and palliative care during the first two years of medical school (84\% in favor, $16 \%$ not in favor; $\mathrm{t}=8.69$, $\mathrm{p}<0.0001)$. They reported interest in learning via early, hands-on clinical experience. Pre-clinical students reported having insufficient knowledge about hospice and palliative care as compared to students in their clinical years $(26 \%$ for pre-clinical students as compared to $74 \%$ for clinical students; $t=3.77, p=0.318$ ). Finally, having clinical experience in palliative and hospice care during medical school improved knowledge of these fields among students $\left(\chi^{2}=19.321, \mathrm{p}<0.001\right)$.

Conclusion: The present study demonstrated that students are interested, willing, and ready to learn about palliative and hospice care medicine during the first two years of medical school. Furthermore, education in these subspecialties proves worthwhile and beneficial for the students. Thus, we recommend integrating more hands-on experience in palliative and hospice care during the first two years of medical education.

29. Creating a Palliative Care Service in a Community
Hospital
Houston Methodist St. John
Nassau Bay, TX
Team/Contact Information:
Katherine Walsh, MSN, DrPH, RN, NEA-BC
Vice President and Chief Nursing Officer
Houston Methodist St. John
18300 St. John Drive
Nassau Bay, TX 77058
(281) 333-8813

kewalsh@houstonmethodist.org

Project Team Members:

Cynthia Broussard, RN

Katherine Walsh, MSN, DrPH, RN, NEA-BC

Topic: Staffing models

\section{Description:}

Creating a Palliative Care Service in a small, community hospital is a challenge. Patient volumes may be low making the allocation of expert staff and other resources a secondary priority. Houston Methodist St. John Hospital, a 177 bed community hospital, part of the Houston Methodist System was able to successfully create, sustain and grow such a service over the past five years by building on the credibility of a few key staff members. Initially, a half time $\mathrm{RN}$ position was allocated to develop and run the program. An ICU RN with interest in palliative care was selected as the director and completed formal training. She garnered support from the Chief Nurse, the Chief Executive Officer and the Chaplain. The credibility and expert communication style of the director led authority to the emerging program. Physicians respected her assessment of patient need and recommendations for plans of care. She slowly enlisted the participation of other intradisciplinary staff and enrolled them in formal training. A
Chaplain and Speech Therapist began seeing patients in addition to their usual responsibilities. Over time, a pharmacists with expert advocacy skills, a social worker in the case management department with knowledge of community services, became involved. Team members began meeting one on one with physicians regarding services. The program was formalized with a strategic plan, creation of patient and staff educational programs and the implementation of a patient needs assessment. Two private, palliative care trained physicians serve as resources for the service and the attending physicians. The original director now serves in a full time capacity and has a staff of two Registered Nurses in addition to the intradisciplinary team members that serve from their home units. To capitalize on resources, this team also serves as patient liaisons and manages patient and family concerns. The metrics validate the success of this service; the volumes have grown year over year with a $6.6 \%$ palliative care penetration (quartile 4 of 2014 CAPC database). The mean annual initial inpatient visits per FTE also is in the fourth quartile. The LOS pre palliative care consult exceeds the fourth quartile, indicating the acceptability of this service and is a testament to the talent and skill of the team members. Consult to discharge and total LOS also exceed the fourth quartile. In summary, the power of one, credible, passionate leader with the ability to enlist and engage others can develop a successful service utilizing the resources at hand. As success builds upon success, the growth of the service occurred and is leading the benchmarks with its outcomes. Such a service is not typical of a small organization, yet the impact on the quality of patient care and LOS is significant.

\section{Characterizing Quality in Inpatient Palliative Care}

Icahn School of Medicine at Mount Sinai

New York, NY

Team/Contact Information:

Ian B. Kwok, BA

Icahn School of Medicine at Mount Sinai

1 Gustave L. Levy Place

New York, NY 10029

(212) 241-6500

ian.kwok@mssm.edu

Project Team Members:

Ian B. Kwok, BA

Maggie Rogers, $\mathrm{MPH}$

Topic: Measuring impact and value

Description:

Background: Over two-thirds of hospitals in the United States have palliative care programs, including $90 \%$ of hospitals with 300 or more beds. However, there is little data assessing the characteristics of these programs and how differences in program quality may affect patient care.

Objective: To identify common characteristics of inpatient palliative care programs, and to explore their effects on hospital outcomes.

Methods: Program-specific data from the National Palliative Care Registry were compared with hospital-level outcomes obtained from the Dartmouth Atlas of Health Care (20132014). Multivariate logistic regression analyses were used to identify program characteristics that were significantly associated with relevant patient outcomes.

Preliminary Results: Of 219 inpatient palliative care programs (based in US hospitals with 300 or more beds) included in this study, 107 (48.9\%) reported staffing a complete interdisciplinary team (as defined by the Joint Commission) and 80 (36.5\%) reported high levels of ICU integration. The presence of a complete interdisciplinary team was associated with a 3.23 
$(\mathrm{p}=0.019,95 \% \mathrm{CI}=0.547-5.916)$ decrease in the percentage likelihood of an ICU admission and a decrease in mean ICU days (intermediate intensity) from 2.57 to 1.98 ( $p=0.039,95 \%$ $\mathrm{CI}=0.030-1.157)$ in the last 6 months of life. In addition, programs reporting high levels of ICU integration were associated with a $2.83(\mathrm{p}=0.028,95 \% \mathrm{CI}=0.312-5.355)$ decrease in the percentage of patients with a primary care visit within 14 days of discharge to home and a $0.58(\mathrm{p}=0.027,95 \% \mathrm{CI}=0.066-$ 1.085) decrease in the percentage of patients with an emergency department visit within 30 days of discharge to home.

Discussion: The preliminary results suggest that the presence of a complete inpatient palliative care interdisciplinary team and high levels of ICU integration are significantly associated with improved hospital-level outcomes, despite relatively low penetration. The wide range of different staffing and integration models across US palliative care programs suggest a need for further investigation into program quality measures and their potential effects on patient care.

31. Night and Day: A 24-Hour Coverage Solution for a PCU Icahn School of Medicine at Mount Sinai Hospital New York, NY

Team/Contact Information:

Bridget Tracy, $M D$

Assistant Professor

Icahn School of Medicine at Mount Sinai Hospital

1 Gustave L. Levy Place, Box 1070

New York, NY 10029

(212) 241-1446

bridget.tracy@mssm.edu

Project Team Members:

Bridget Tracy, $M D$

Maria Reyna, MD

Emily Chai, $M D$

Topic: Staffing models

Description:

Objectives: Outline the challenges of covering a primary service with 24 hour demands, but only 12 hour coverage and describe a staffing solution that builds on partnerships with hospitalist colleagues.

Background: Our Palliative Care Unit (PCU) is a 14-bed geographically distinct unit in the Department of Geriatrics and Palliative Medicine which opened in June 2011. We accept patients with complex symptom management needs, who require support for establishing goals of care, or who have chosen comfort-oriented care. The PCU is staffed during the day by specialty trained palliative care (PC) physicians, nurse practitioners (NP), social workers, bedside nurses, patient care associates, and a clinical nurse manager. Caring for patients with 24-hour needs when the unit has no nighttime staffing presented a significant challenge.

To illustrate this problem, consider the case of TA, a 70 year old woman with gastric cancer metastatic to lung, presenting from the Emergency Department with dyspnea. She was evaluated by our team and her goal was to be comfortable and return home with hospice. The PC consult team ordered morphine which relieved her dyspnea after two doses, and began a scheduled morphine regimen. She was transferred to our PCU and evaluated as comfortable by the PC physician and NP at 4pm. At midnight, however, she became acutely dyspneic, hypotensive, hypoxic, and tachycardic.

Staffing Solution: Our team collaborated with the Division of Hospital Medicine within the Department of Medicine to provide seamless, continuous coverage overnight for our acutely ill and often symptomatic patients. Given their unit's proximity to our floor, two physician assistants (PA) were se- lected to cover the PCU, guaranteeing a timely response to patients' and families' needs. To ensure quality coverage, the PAs rotated with the PC service for 3 days, observed family meetings, and received intensive education on pain and symptom management. In addition, we established back up by pager from both a palliative provider, as well as an in-house hospitalist. The PCU team sends a daily handoff email to the PA listing code status, contact for family or decision makers, cause of death, clinicians to notify at time of death, and a brief clinical summary for each patient. This email can be quickly referenced when the PA responds to patients' changing clinical status, needs to update families, or has to fill out a death certificate.

With this staffing solution in place, TA was immediately evaluated by the covering PA, who ordered additional morphine and updated her husband. The PA also paged the PC fellow on call after the dose of morphine did not alleviate the patient's dyspnea. Together, they developed a new regimen that relieved TA's symptoms. TA died comfortably a few hours later, and was pronounced by the PA.

Conclusion: Providing intensive palliative care 24 hours a day is challenge for rapidly growing programs. Partnerships within the hospital can help to ensure the timely delivery of high quality round the clock care to patients while also advancing primary palliative education.

\section{Reducing Readmissions through Communication of} Teams

Inova Health System

Inova Fair Oaks Hospital

Fairfax, VA

Team/Contact Information:

Lynne B. Kennedy, PhD

Director Palliative Care Inova Health System

Inova Health System

Inova Fair Oaks Hospital

Service Line Office

3600 Joseph Siewick Drive

Fairfax, VA 22033

(703) 391-3685

lynne.kennedy@inova.org

Topic: Care transitions

Description:

The Palliative Care teams at each of our Health System's Operating Units are at different stages of development. As data was gathered we recognized an opportunity to develop team integration with clinical and medical staff at each in-patient location and incorporate improved communication with the different levels of community care to reduce unplanned patient re-admissions. Handoffs between service lines were improved through direct communication. Handoffs into the community were developed within our electronic health record (EHR) function so that our community partners could view the charts of their patients following discharge from acute care. They would be able to see the levels of Goals of Care conversations that had already transpired and could pick up the conversations from the relevant point of planning rather than initiating the discussion from the beginning again.

Physicians, who had previously consulted the PC team later in the trajectory of the patient's illness began considering earlier referrals. Physicians who had not considered the team's intervention, began thinking about involving palliative providers. The result of this systematic approach led to a consistent growth in PC referrals, incorporation of PC as a "standard of care." The positive impact on hospital data indicated improved pain control; nursing satisfaction with Interdisciplinary Team support and interdisciplinary co-operation strengthened the program. 
The hospitalists, who were getting monthly interactions during their staff meetings, increased referrals. The hospitals, where PC programs were just starting, improved readmission rates but the length of stay was slightly longer, reinforcing the belief that early referrals, accomplishes both reduced length of stay, reduced readmissions, and subsequently lower healthcare cost.

\author{
33. Palliative Screening to Positively Improve Patient Care \\ Inova Health System \\ Inova Fair Oaks Hospital \\ Fairfax, VA \\ Team/Contact Information: \\ Lynne B. Kennedy, PhD \\ Director Palliative Care Inova Health System \\ Inova Health System \\ Inova Fair Oaks Hospital \\ Service Line Office \\ 3600 Joseph Siewick Drive \\ Fairfax, VA 22033 \\ (703) 391-3685 \\ lynne.kennedy@inova.org \\ Project Team Member: \\ Vera Dvorak, $M D$ \\ Topic: Health system strategies \\ Description:
}

In June 2015 we began 100\% screening throughout our Health System by RNs on admission to Medical, Oncology, ICU, and Telemetry units. As data was gathered we recognized an opportunity to do a second screening on patients who had not yet been referred to palliative care (PC). Case Managers now re-screen when the patient's health status changes or the level of care needs to be upgraded. The screening tool in our Electronic Health Record (EPIC), provided multiple methods of requesting a consult order from the Attending physicians at our organization. The most effective method proved to be the nurse calling or speaking directly with the doctor to identify the value indicated by the positive screening. The nurses felt empowered. We worked with them to use "Situation, Background, Assessment, and Recommendations" (SBAR) techniques to communicate the need for a consult. Physicians, who had previously consulted the PC team later in the trajectory of the patient's illness began considering earlier referrals. Physicians who had not considered the team's intervention, began thinking about involving palliative providers. The result of this systematic approach led to a consistent growth in PC referrals, incorporation of PC as a "standard of care." The positive impact on hospital patient satisfaction surveys; improved pain control; nursing satisfaction with Interdisciplinary Team support and cooperation strengthened the program. The hospitalists increased referrals. The hospitals where the programs had already been enculturated progressed more rapidly, both improving length of stay, and lower readmission rates. The hospitals, where PC programs were just starting, improved readmission rates but the length of stay was slightly longer. This result reinforced our belief that early referrals, which occur once enculturation is established, accomplishes both reduced length of stay, reduced readmissions, and subsequently lower medical treatment cost.

34. The System Dashboard for Operational Administration Inova Health System

Inova Fair Oaks Hospital

Fairfax, VA

Team/Contact Information:

Lynne B. Kennedy, PhD

Director Palliative Care Inova Health System
Inova Health System

Inova Fair Oaks Hospital

Service Line Office

3600 Joseph Siewick Drive

Fairfax, VA 22033

(703) 391-3685

lynne.kennedy@inova.org

Project Team Member:

Vera Dvorak, $M D$

Topic: Measuring impact and value

Description:

Implementation of a system dashboard as a comprehensive database for operational administration using a customized palliative care flow chart built into our electronic health record. In order to identify opportunities to develop team integration with clinical and medical staff at each of our hospitals, and form a more dynamic interdisciplinary group with a focus on improved patient care. Utilization outcomes were measured in terms of pre and post length of stay, readmissions, and palliative penetration. The data reassessment led to the creation of more efficient programs such as timing of the patient screening and consultation, ongoing education of the staff and process changes.

35. Implementation of a Pediatric PC Champions Program Johns Hopkins All Children's Hospital

St. Petersburg, FL

Team/Contact Information:

Michelle Cook, MS

Advanced Education Specialist

Johns Hopkins All Children's Hospital

501 6th Avenue South

St. Petersburg, FL 33701

(727) 767-8275

michelle.cook@jhmi.edu

Project Team Members:

Kristina Burger, DNP, ARNP, CPNP, CCRN, RN-BC, LNC

Laura Drach, DO, MSN, FAAP

Karen Hansen, BS, CCLS, GC-C

Marclaire McEachern, RN, CPN, CHPPN

Susan Shields, MSN, ARNP, CPNP

Michelle Cook, $M S, R N, R N-B C, C H P P N$

Topic: Palliative care education

Description:

Introduction: Caring for a child with a life-limiting illness can be one of the most difficult parts in the role of a healthcare professional (HCP). Palliative care has been shown to relieve suffering across multiple realms, including physical, psychological, practical and existential; improve quality of life; facilitate informed decision making and assist with coordination of care (American Academy of Pediatrics, 2013). Provision of quality palliative care includes acquisition of knowledge, skills, and attitudes in a variety of critical concepts including communication; pain and symptom management; care at the time of death; loss, grief, and bereavement; and ethical and legal issues (ELNEC-PPC, 2013). However, HCPs often admit a lack of comfort and confidence in providing these critical concepts when a patient is diagnosed with a life-limiting illness or at the end-of-life.

This poster will present an overview of an innovative pediatric Palliative Care Champions Program. In addition, the poster will provide an outline of the program eligibility, initial and ongoing education, and maintenance requirements. Furthermore, we will identify potential benefits of the program for patients/families, staff, the champions, and the Palliative Care 
Team. Finally, an overview of outcome measurement and preprogram data will be revealed.

Program Purpose: Initiation of a Palliative Care Champions Program will improve the quality of care provided to patients \& families diagnosed with life-limiting illnesses, receiving palliative care, or at end-of-life.

Program Objectives: •Promote comprehensive quality palliative care for patients across the healthcare system $\bullet$ Enhance the collaboration that occurs between unit staff \& the Palliative Care Team $\bullet$ Select staff members with an interest in palliative care to serve as unit champions $\bullet$ Develop an educational program to train \& provide ongoing support and education to the unit champions.

Role of the Champion: 1.To function as a resource for unit staff related to palliative care, end-of-life care, and quality of life 2.To serve as a liaison for staff $\&$ family needs 3.To improve the quality of care provided to patients \& families diagnosed with life-limiting illness or at end-of-life.

This hospital has successfully trained 34 Palliative Care Champions. These champions function as a resource for staff related to palliative care and quality of life. The champions also mentor and educate staff on palliative care topics, while advocating for patients who are diagnosed with life-limiting illnesses or at end-of-life. In addition, the champions meet quarterly for an educational topic and to share ideas, successes, and frustrations. These Palliative Care Champions are passionate and engaged, and are striving to improve the quality of care that patients and families receive.

\section{Enhancing Rural Hospice Care with Mobile Tablets KU Center for Telemedicine \& Telehealth \\ Fairway, KS \\ Team/Contact Information: \\ Eve-Lynn Nelson, PhD \\ Director \& Professor \\ $K U$ Center for Telemedicine \& Telehealth \\ 4330 Shawnee Mission Parkway, Suite 136, MS 7001 \\ Fairway, KS 66205 \\ (913) $588-2413$ \\ enelson2@kumc.edu}

Project Team Members:

Gary Doolittle, MD

Sandy Kuhlman, RN, BSN

Eve-Lynn Nelson, PhD

Ashley Spaulding, MA

Hope Krebill, RN, BSN, MSW

Topic: Leveraging technology (e.g., EMR, telehealth)

\section{Description:}

In rural communities, hospice providers often travel great distances to reach patients across expansive service areas, resulting in unique challenges to maintain quality as funding decreases. That travel raises concerns around cost-effectiveness, access, and safety. For hospice visits in which the patient could have been evaluated and supported using technology just as effectively as an in-person evaluation, the travel time and associated costs are unnecessary expenses to the healthcare system. Safety becomes an issue when hospice providers have to travel in dangerous weather conditions. A TeleHospice $(\mathrm{TH})$ service utilizing low-cost, secure mobile tablets (iPads) has the potential to support family-centered, timely care directly to patient homes. This academic-community partnership aims to enhance palliative care capacity in Kansas' rural and frontier communities.

The University of Kansas Medical Center (KUMC) piloted the country's first TH service in 1998, with technology limita- tions, attitudes towards technology, and costs limiting broad adoption at that time. Leveraging lessons learned from this early work, an interdisciplinary team adapted the TH home-based approach for mobile tablet technology. A secure cloud-based videoconferencing solution was chosen for ease of use. Selection of hospice partners for the pilot was guided by Gustafson et al.'s (2003) Organizational Change Manager (OCM) implementation survey.

Based on OCM feedback from 16 staff, Hospice Services, Inc. (HSI), a not-for-profit community-based hospice serving 16 rural Kansas' counties, was selected to partner with KUMC to implement TH. Overall, responses indicated positive attitudes towards TH. Three-fourths of the respondents believe TH will save travel time and enhance their job. A majority (73.3\%) believe TH will help HSI meet its goals, and $81.3 \%$ believe the project will help meet staff needs. Respondents overwhelmingly indicated that they know how the project will connect the hospice medical director with the clinical team at patient homes $(81.3 \%)$, how it will connect clinical staff with each other $(86.7 \%)$, and how it will connect clinical staff with families in their homes $(81.3 \%)$. Nearly $90 \%$ believe that HSI leaders have endorsed $\mathrm{TH}$ in visible ways, and $75 \%$ feel that a clear project aim has been specified.

The KUMC and HSI teams meet regularly via phone and videoconferencing to collaborate on project development, implementation, and evaluation. Key implementation considerations have included: initial and ongoing training, protocols, ongoing technical/administrative support, and introductory TH information. Data further indicate that HSI personnel utilize the iPads to connect in many ways, such as meetings and presentations. TH connections have allowed hospice nurses at the home to videoconference with additional HSI personnel, including the medical director as well as social workers. Eventually, iPads will be utilized in patient homes to enhance hospice care through symptom assessment and support for both patients and caregivers. These technology-based assessments will help hospice nurses determine if an in-person visit is necessary, which has the potential to reduce travel and other costs. Though TH visits are not intended to replace traditional hospice visits, TH's potential to supplement hospice services and improve hospice care while reducing hospice costs is significant.

\section{Palliating the Palate: Making Oral Care a Vital Sign Lee Memorial Health System} Ft Myers, FL

Team/Contact Information:

Amber Greco, MSN

Lee Memorial Health System

8931 Colonial Drive

Ft Myers, FL

(239) 343-2000

asgreco@eagle.fgcu.edu

Topic: Palliative care education

\section{Description:}

Oral pharyngeal candidiasis is a leading cause of death in patients with advanced cancer. Risks to oral care in patients with advanced disease are great, and include but are not limited to poor oral intake, debility, local radiation, medications, and chemotherapy. So why do most providers spend such little time doing a thorough oral examination and diagnosis? The standard care still remains a quick peek in the mouth and most patients receive the same initial cocktail, the "magic mouthwash." Wiseman a palliative care dentist observes oncologists treat patients with multidrug concoctions while evidence supports 
remedying the specific symptoms (2006). Quelling the storm and restoring the oral biome supports the design of patient comfort. Candida should be cultured for sensitivity, evidenced by $52 \%$ of terminal patients in a Denmark study whom were colonized by a yeast with reduced susceptibility to Fluconazole (Astavad et al. 2015). Patients with periodontal inflammation have shown recovery when treated with local culture supernatant Lacidophilus Strain (Haukioja, 2010).Probiotic engineering and local application show promise to aid in restoring oral biome in future. Mouthwash solutions can be beneficial to restoring the calm, and there are multiple simple options which should be chosen according to the palliative patient's specific tastes and needs. Water and saline solution are easily accessible but saline can alter taste. Of cardinal concern is a dry mouth, there is a need to cleanse but preserve moisture. A simple solution of 1:1 cider to soda water is palatable and effervescence debrides well (Davis and Finlay, 2005). It is necessary two moisturize mouth minimally every two hours, especially at the end of life a large cotton soaked swabs are a good choice, often if patient semiconscious ice water can be unsettling. There is even suggestion of liquor to swab mouth which can calm patient who has been used to drinking alcohol. Oral pain is unacceptable, after dry mouth is addressed, use of numbing agents or oral Morphine $0.2 \%$ solution should be considered. Dentists can be invaluable members to a palliative care team, but the reality for most patients' with advanced disease is poor dental care and reduced access to a dentist. The oral health of our palliative patient must be considered a vital sign and treated with the same urgency as their other organ systems. A is for airway in the $\mathrm{ABCs}$ of resuscitation, so let us remember to attend the oral pharyngeal system.

\section{References}

Astavad K, Johansen HK, Hoiby N, Steptoe P, and Ishoy, T. JPalliative Med.2015:18(11).

Davis A. Finlay I. Oral Care in Advanced Disease, Oxford University Press, London, 2005.

Haukioja A. Probiotics in Oral Health. EurJDent. 2010:July 4(3) Wiseman MA. Palliative care dentistry.JCanDentAssoc.2006: 72(5).

\section{PC \& OPO Collaboration Ensures Patient-Centered EOL Care}

Grady Health System

Atlanta, GA

Team/Contact Information:

Paul DeSandre, DO

\section{Director}

Grady Health System

80 Jesse Hill Jr Drive SE

Atlanta, GA 30303

(770) 225-5465

pdesandre@emory.edu

Project Team Members:

Paul DeSandre, DO

Kimberly Kottemann, MBA, LifeLink of Georgia

Topic: Integration of palliative care to specific settings (e.g., hospital, ICU, home, nursing home, clinic)

\section{Description:}

Background: Inpatient hospice services within hospitals serve as an effective care option for patients in critical condition who no longer benefit from critical care interventions, and in whom the goals support a transition to comfort. Without effective collabo- ration, patients may be removed from life-sustaining interventions prior to considering an opportunity for organ donation. Palliative care $(\mathrm{PC})$ provides an essential bridge between organ procurement organizations (OPO), primary medical teams, and the patientfamily unit in assuring optimal end-of-life (EOL) care.

Objective: To determine the influence of a PC and OPO collaboration on organ donation and inpatient hospice services.

Methods: In April 2015, our institution began inpatient hospice services for physiologically unstable patients. In August 2015 , our PC team established a collaboration with our OPO to ensure that goals for end-of-life care include the possibility of organ donation. The OPO notified the PC team of all new referrals and provided updates at key process points. The PC team screened new consults meeting OPO triggers to ensure referral. A flowchart was used to outline and assure bilateral communication.

\section{Results:}

\begin{tabular}{lccc}
\hline & $\begin{array}{c}\text { Nov 2014- } \\
\text { Apr 2015 }\end{array}$ & $\begin{array}{c}\text { May 2015- } \\
\text { Oct 2015 }\end{array}$ & $\begin{array}{c}\text { Nov 2015- } \\
\text { Apr 2016 }\end{array}$ \\
\hline Organ Referrals & 132 & 140 & 148 \\
Organ Donors & 10 & 10 & 16 \\
PC Referrals & 450 & 635 & 779 \\
Hospice & 18 & 160 & 194 \\
$\quad$ Admissions & & &
\end{tabular}

Conclusion: We demonstrate that a PC and OPO partnership created synergistic improvements in all services during a growth phase of the program development of PC and hospice at our institution. Through a cooperative protocol, we were able to assure that the values and needs of the patient and family were properly explored and honored.

Our results support an important leadership role for PC in establishing policies and practices that encourage collaboration with the OPOs to assure optimal end-of-life care.

39. Implementing Virtual Hospice: Challenge and Discovery Medical College of Wisconsin

Milwaukee, MI

Team/Contact Information:

Wendy L. Peltier, MD

Associate Professor

Medical College of Wisconsin

9200 West Wisconsin Avenue

Milwaukee, MI 53226

(414) 805-4607

wpeltier@mcw.edu

Project Team Members:

Wendy L. Peltier

Liza Thiel

Jessica Lisinski

Molly Kast

Colleen McCracken

Susan Hoefs

Sandy Simuncak

Kathy Walczak

Sandy Muchka

Tiffany Kirchner

Topic: Integration of palliative care to specific settings (e.g., hospital, ICU, home, nursing home, clinic)

\section{Description:}

Implementation of a virtual hospice service in a large academic center: Challenges, solutions and discoveries. 
Objective: Design and implementation of a virtual hospice service as a bridge to future inpatient unit.

Methods: Strategic planning with hospital and health system leadership identified a gap in inpatient hospice services. Plans for an inpatient unit were significantly delayed due to hospital capacity constraints. Creation of a virtual hospice program was requested and aligned with the palliative care team and a respected local hospice agency. Key elements included palliative care as the primary attending service, patients formally enrolled in the hospice benefit, room enhancements and 24/7 hospice team support.

Results: Implementation was staged, starting in medical and neurologic intensive care units, pre- selected by administration due to high mortality rates. In the first seven months, with rapid roll-out, 91 patients and families were cared for, with an average length of stay of 1.0 days. Ten patients died while still in the ICU, 75 were transitioned to a ward bed and six patients were discharged. Hospital mortality index declined by $36 \%$. Challenges included: investment of non-clinical time to develop the program, lack of buy-in from multiple sectors (hospital RN's, case management, referring MDs, palliative MDs) about potential benefit to patients and families, computer workflows, cost and workforce concerns. Several key quality improvement areas were identified during implementation, including provider lack of knowledge regarding the difference between hospice and palliative care, as well as varying practices in opioid dosing during terminal extubation.

Conclusions: Virtual hospice services have the potential to enhance end-of-life experiences for patients and families with anticipated hospital death, but provide significant challenges in implementation. Key factors in the initial success of our hospice program include: tremendous support from hospital and hospice agency senior leadership, multi-disciplinary planning, flexibility in palliative medicine faculty and nurse practitioners, identification of anticipated cultural challenges prior to implementation and 24 hour support from experienced hospice RNs.

40. Field of Dreams: Rapid Expansion of PC in a Health
System
MedStar Health
Baltimore, MD
Team/Contact Information:

Team/Contact Information:

Kathryn A. Walker, PharmD

Senior Clinical and Scientific Director of Palliative Care, Associate Professor

MedStar Health

201 E. University Pkwy, 33rd St Bldg

Baltimore, MD 21218

(410) 375-3699

kathryn.walker@medstar.net

Topic: Health system strategies

Description:

"Build it and they will come"... Starting a program in a single hospital within a healthcare system has challenges, but spreading PC (Palliative care) across a diverse healthcare system is another level of challenge on a wide scale level. When quality programs begin having outcomes that gain executive level attention, it becomes evident that there is a price to not growing PC programs. We sought to expand PC services across our ten hospital health system after demonstrating outcomes at one site. Our staffing model includes a standard transdisciplinary team model of physician, nurse practitioner, clinical pharmacist, social worker and chaplain at each site. Within two years, our program grew from 4 hospitals to all ten having programs demonstrating valuable outcomes, sharing best practices, leveraging research projects and working together through discipline-specific clinical councils. This data will reflect the growth over three fiscal years of having a system wide PC service and describe the model for benchmarking quality measures. Overall, our programs have an average of $32 \%$ reduction in daily charges compared with pre-PC daily charges. This represented an average decreases in daily charges in the following categories: $33 \%$ in medication charges, $53 \%$ in laboratory charges, $28 \%$ in room charges. We demonstrated early PC consultation ( $\leq 4$ days) was associated a $32 \%$ decrease in length of stay compared to patients with severe/extreme risk of mortality and severity of illness with palliative care coding. Readmission data will be presented per hospital and represent a range of impacts on 30 day all cause readmission compared to other patients with severe/extreme risk of mortality and severity of illness in the hospitals. Penetration and benchmarking for each hospital will be presented.

\section{PATCH2 Program: The Creation of a Virtual Palliative Care Clinic}

MedStar Health

Baltimore, MD

Team/Contact Information:

Kathryn A. Walker, PharmD

Senior Clinical and Scientific Director of Palliative Care, Associate Professor

MedStar Health

201 E. University Parkway, 33rd Street Building

Baltimore, MD 21218

(410) 375-3699

kathryn.walker@medstar.net

Project Team Members:

Nicole Graham, PharmD

Dave Brennan

Hunter Groninger, MD

Renee M. Holder, PharmD

Kasey Malotte, PharmD

Leigh Cervino - Pharmacy Student

Andre D'Souza - Research Intern

Christopher Kearney, MD

Topic: Leveraging technology (e.g., EMR, telehealth)

Description:

Background: We often lose touch with our patients after spending a lot of time connecting with them during their hospital stay. To prevent this lapse in care, we will describe how we harnessed the power of video conferencing and mobile technology to redefine the boundaries of our team and provide effective, efficient patient care outside the walls of our community teaching hospital. This project represents collaboration between palliative care, home health and telehealth to extend palliative care outside the walls of the hospital to provide continuity of care. Through the smart and appropriate use of mobile technologies we aimed to improve patient/provider communication, facilitate a comprehensive approach to medication management, and enhance patient self-care education in a target population of urban-dwelling patients with advanced heart failure admitted to one of four participating hospitals.

Methods: Patients are approached for consent if a PC consultation is ordered by the attending physician, the patient has a diagnosis for advanced COPD or heart failure (stage C or D), and the patient is planned to be discharged home. Once 
consented, the patients are randomized (2:1) to receive PC follow up after hospital discharge through either an internet-equipped electronic tablet or telephone. Patients who do not wish to be enrolled in the study receive normal follow up post discharge and data for this group will be collected through a retrospective chart review to compare readmission rates between groups. Both groups of study patients receive PC team follow up meetings (via video conference or phone call) post discharge at 5, 15,30, and 60 days. Patients self report symptoms, quality of life, satisfaction with care, communication, and medication adherence using surveys. Tablets also provide patients with updated medication lists, targeted education, and a recording of their family meeting to review with family members. Primary outcomes will include 30 day readmission data compared to 30 days pre-program enrollment and medication interventions. Medication interventions are reported as patient safety events prevented and categorized using NCC MERP (National Coordinating Council for Medication Error Reporting and Prevention) scale.

Results: Sixty patients were enrolled during the first 18 months with an average age of 60 years (range 30-91). Compared to pre-PATCH2 enrollment, hospital admissions were decreased by $60 \%$. Medication interventions were categorized as patient safety events prevented and $9 \%$ would have reached the patient and caused harm, 53\% would have reached the patient and required additional monitoring/intervention, and $13 \%$ would have reached the patient without harm.

42. Virtual Palliative Clinic for LVAD Patients at Home
Medstar Washington Hospital Center
Washington, DC
Team/Contact Information:
Andre D'Souza
Research Coordinator
Medstar Washington Hospital Center
110 Irving Street NW
Washington, DC 20010
(856) 381-7403
asdsouza95@gmail.com
Project Team Members:
Renee M. Holder, PharmD
Andre D'Souza
Nicole Graham, PharmD
Hunter Groninger, MD
Selma Mohammed, MD
Kathryn A. Walker, PharmD
Topic: Specific patient populations
Description:

Background: Given the severity of illness with LeftVentricular Assist Device (LVAD) implantation, it is clear that LVAD patients seen by the PC team while hospitalized could benefit from more frequent out-of-hospital healthcare interaction. Telehealth can provide a link for these patients to provide clinical support without the burden of transportation and energy requirements for in-person clinic visits. To this end, the Palliative Telehealth Connecting Hospital to Home (PATCH2) Program was created as a "virtual clinic" for advanced heart failure patients following hospitalization. This represents a case study of the first LVAD patients to enroll in this program.

Objective: To describe the impact of telehealth visits on the post-hospital care of a cohort of LVAD patients with palliative needs. Methods: Patients with LVADs and receiving PC consultation with a discharge plan to home, between January and March 2016, provided informed consent, randomized and enrolled to tablet or telephone groups for follow up. Patients were excluded if they could not report their own symptoms, or did not speak English. Both groups of study patients will receive PC follow up meetings from the PC clinical pharmacist and social worker (via video conference or phone call) post discharge at 2-5, 15, 30, and 60 days. Recommendations and updates are communicated to the primary medical teams. Patients in both groups completed surveys to report symptoms, quality of life, satisfaction with care, communication, and medication adherence. Tablets will also provide patients with updated medication lists, and targeted education. This study was approved by MedStar Health IRB.

Results: To date, this case series includes the first five patients to complete the first month in the PATCH2 program (2 tablet, 3 phone). Eleven patients were enrolled, however 6 had barriers to participation: not able to reach patient (3/11), readmitted to subacute rehabilitation/hospitalizations (2/11), and one requested removal from program before initial contact. The case study patients $(n=5)$ had a mean age of 59 years (range 46-70), 3/5 were male and 3/5 were African American. Two patients were enrolled during the index admission for LVAD implantation. The reasons for PC consultation were pain $(2 / 5)$, non-pain symptoms (2/5), DT-LVAD evaluation (1/5), and goals of care (1/5). For this session, an updated series summary of patient demographics, clinical interventions made during $\mathrm{PATCH} 2$ visits along with responses reflecting symptom scores, satisfaction with care, and quality of life will be presented.

Conclusions: Patients with heart failure requiring an LVAD have potential to benefit from prolonged palliative care interactions. A model for palliative care telehealth visits is described, as well as challenges related to engaging patients.

\section{Expanding Practice: PharmD and SW Led Palliative Consults \\ Medstar Washington Hospital Center \\ Washington, DC \\ Team/Contact Information: \\ Renee M. Holder, PharmD \\ Clinical Pharmacist, Palliative Care \\ Medstar Washington Hospital Center \\ 110 Irving Street $N W$ \\ Washington, DC 20010 \\ (202) 877-7841 \\ renee.m.holder@medstar.net \\ Project Team Members: \\ Regina Tosca, LICSW \\ Anne Kelemen, LICSW \\ Hunter Groninger, $M D$ \\ Topic: Measuring impact and value \\ Description:}

Background: Palliative care (PC) teams often rely on traditional medical providers to lead consultative encounters, potentially delaying access to PC expertise and preventing other disciplines from practicing fully within their scope. To illustrate an alternative approach, we describe a series of PC consultations served well by expanded team roles.

Methods: PC consultations for a single center were reviewed from January to May 2016. Patients seen by both a pharmacist (PharmD) and social worker (LICSW) but not a physician or nurse practitioner in the first 72 hours of consultation were considered for inclusion. PC consultation metrics and chart notes from PC team members were reviewed for common actions and themes.

Results: Eight patients met inclusion criteria. Palliative diagnoses were: cancer, solid (5); heart failure (3). Reasons for 
consultation included: advance care planning (ACP) only (4); pain only (2); ACP and pain (1); ACP and non-pain symptoms (1). Setting of initial consultation was medical/surgical floor (3); cardiac floor (2); intensive care unit (1); intermediate care unit (1); emergency department (1). Setting of discharge included: home (4); death (2); acute rehabilitation facility (1); home hospice (1). PharmD activities included: medication initiation, titration and monitoring; opioid risk screening; substance abuse screening and counseling; symptom identification and treatment; medication reconciliation; deprescribing; family meetings. LICSW activities included: psychosocial assessment and counseling; completion of advance directive; family meetings; nonpharmacologic pain management; reiki; bereavement counseling. Two patients were seen by a physician or nurse practitioner $>72$ hours after initial consultation. In those cases, recommendations were in concordance with LICSW and PharmD, and advancements in ACP and were achieved in one case based on preliminary discussions held by LICSW and PharmD with patient and family. No adverse outcomes of recommendations or interventions were noted to occur during hospitalization.

Conclusions: Non-physician palliative care team members, such as clinical pharmacists and social workers, can provide skilled symptom management and advance care planning in various settings, palliative conditions, and disease stages. Enabling PC team members to work fully within their scope of practice expands the team's clinical reach and accelerates bedside access to PC expertise.

44. Palliative Care Wherever the Patient Is ... Everywhere! Mercy Regional Medical Center

Durango, CO

Team/Contact Information:

Daniel Keuning, RN MSN FNP-C ACHPN

Palliative Care Coordinator

Mercy Regional Medical Center

1010 Three Springs Boulevard

Durango, CO 81301

(970) 382-2000

dankeuning@yahoo.com

Project Team Members:

Daniel B. Keuning, RN MSN FNP-C ACHPN

Anne Rossignol, MD

Topic: Integration of palliative care to specific settings (e.g., hospital, ICU, home, nursing home, clinic)

\section{Description:}

Providing Palliative Care in multiple patient care settings from ER to ICU to Med Surg, then to SNF and Rehab, Specialty clinics and ultimately home. Reducing acute care readmission rates to less than $2 \%$ by following patients to multiple care settings with palliative care NP and MD services. Caring for patients in a 85 bed rural hospital with a dedicated team of MD/ NPs, MSW, Chaplains, and Case Management focused on the patient and family needs. Transitioning appropriate patients to hospice and increasing hospice length of stay from 18 days to 45 days in 1 year. Collaborating with specialists by providing palliative care consults in their offices has increased collaboration with care for the most difficult cases. Changing the culture of end of life care in Southwest Colorado has been the result of imbedding palliative care in multiple care settings.

Stats: 85 bed rural hospital. Home Palliative Care provided by 5 NPs. Corporate support by Centura Health to provide education and resources to the hospital and home care. Quality Audits demonstrated greater than $90 \%$ compliance in areas such as advance directives, pain/dyspnea management, bowels, and goals of care discussion. Increased volume of home palliative care visits from 30 per month in 2012 to 250 per month in 2016. Increased staff to include a full time Palliative Care Medical Director in 2016. Four full time NPs providing home palliative care in 3 counties in SW Colorado. Utilizing staff from home health and hospice to transition patients from home care to hospice care seamlessly for patients and families.

\section{Better Outcomes with Palliative Care Staff Education}

Memorial Hermann Physician Network (MHMD)

Houston, TX

Team/Contact Information:

Julie Kendrick, BSN, RN

$R N$ Coordinator

Memorial Hermann Physician Network (MHMD)

909 Frostwood Drive

Houston, TX 77024

(281) 913-4415

julie.kendrick@memorialhermann.org

Topic: Palliative care education

\section{Description:}

Palliative and Supportive Medicine programs are rapidly growing in U.S. hospitals and are assisting them to achieve highquality and well-coordinated medical care. Supportive Medicine is an interdisciplinary specialized team designed to provide an extra layer of support for seriously ill patients and their families. Providing education for hospital staff yields improved patient outcomes for the Supportive Medicine patient. Diane Meier, MD, director of the Center to Advance Palliative Care (CAPC) states, "Education does change behavior, and changes in behavior change outcomes that matter to patients and their families."

Education and training of hospital staff can be achieved by several different platforms. Individual and group training with printed educational resources are effective platforms in Supportive Medicine education. The interdisciplinary team provides individualized education at the bedside creating an educational opportunity, which allows staff to ask questions directly. Group training with nursing units, care management, physicians and therapy staff allows the Supportive Medicine team to provide an informative session. These training classes are scheduled with the hospital's educational department and are presented during quarterly, annual and new employee orientations. PowerPoint presentations, printed pocket guides, brochures, and flyers are used to market the Supportive Medicine team's services and contact information.

Nurses, physicians, therapists, and case management staff have been empowered through education to assess and identify patients that meet criteria for a Supportive Medicine consult. In 2015 consultations received in the first 6 months of operation in the hospital setting with minimal education were 216 . In contrast, 290 consults were received in the first 6 months of 2016 due to a robust educational program, which resulted in a $25.6 \%$ increase. Therefore, effective educational programs benefit hospital staff in recognizing patients needing a Supportive Medicine consult.

\author{
46. An e-App To Foster Symptom Documentation and \\ Intervention \\ Moffitt Cancer Center \\ Tampa, FL \\ Team/Contact Information: \\ Diane Portman, MD, FAAHPM \\ Chair, Department of Supportive Care Medicine \\ Moffitt Cancer Center \\ 12902 Magnolia Drive, MRC-SCM
}


Tampa, FL 33612

(813) 745-7383

diane.portman@moffitt.org

Project Team Members:

Diane Portman, MD, FAAHPM

Sarah Thirlwell, MS, RN, AOCNS, CHPN

Sahana Rajasekhara, MD

Lucy Akins, BSN, RN, CHPN

Topic: Leveraging technology (e.g., EMR, telehealth)

Description:

Background: Assessment of patient-reported outcomes (PROs) has become an important component of health care to gauge the impact of disease and medical treatment on patient well-being. There is need for increased symptom reporting that integrates into the EHR to optimize documentation and prompt intervention. However, collecting PROs during clinic encounters and populating them into the EHR remains challenging. We evaluated the feasibility of implementing a point-of-care electronic PRO assessment via a tablet-based Edmonton Symptom Assessment System (ESAS) application (app) in the palliative care (PC) clinic of our cancer center.

Methods: A multidisciplinary team comprised of members from IT and PC co-developed a tablet-based e-ESAS app. Beginning in January 2016 patients completed ESAS scores in the waiting area prior to each PC clinic visit using the tablet based app. Each symptom element is scored by the patient on an easy-toread tablet page that also includes a brief explanation of the item. Electronic-ESAS scores populate directly into the EHR Flowsheet as vital signs and into the provider's EHR visit note. Thresholds were set for severe scores which are then denoted as high in the Flowsheet and note. Scores can be trended and graphed over time to assess effects of interventions. To determine the impact of the ESAS app, a chart review was conducted of EHR documentation of symptoms and interventions between January to June 2016.

Results: In the initial 4 week period following implementation, $86 \%$ of patients seen in the oncology PC clinic completed symptom reporting via the e-ESAS app. The documentation of symptoms in the EHR was $100 \%$ for encounters for which the app was completed. From January through June, 68\% of visits noted symptom scores exceeding a severity of 6 , with an average of 3 severe symptoms per visit. All visits had documentation of intervention for symptoms, with an average of 5 symptoms addressed per visit. One hundred per cent of records noted provision of counseling or education, $99 \%$ reported medication prescription or adjustments, and $7 \%$ documented referrals to other supportive care specialists.

Conclusion: The high completion rate of the e-ESAS app in our clinic indicates that point-of-care electronic ESAS assessment is acceptable to patients and is feasible to use to capture patient-reported symptoms in the oncology PC setting. The capture of ESAS data via a tablet linked to the EHR resulted in symptom documentation and interventions. Future directions include ESAS app implementation in other oncology clinics and development of prompts to foster intervention for high scores, including PC referrals.

\author{
47. A Palliative Care Summary EHR Page \\ Moffitt Cancer Center \\ Tampa, FL \\ Team/Contact Information: \\ Sarah Thirlwell, RN, MS, CHPN, AOCNS \\ Supportive Care Medicine Director \\ Moffitt Cancer Center \\ 12902 Magnolia Drive
}

Tampa, FL 33612

(813) 745-4673

sarah.thirlwell@moffitt.org

Project Team Members:

Sarah Thirlwell, RN, MS, CHPN, AOCNS

Donna Davis, RN, BSN

Diane Portman, MD, FAAHPM

Topic: Leveraging technology (e.g., EMR, telehealth)

Description:

Introduction: We sought to improve electronic health record (EHR) documentation of inpatient palliative care by creating a page to summarize relevant interdisciplinary clinical information and team care planning. Consistent with national guidelines and The Joint Commission requirements for Advance Certification in Palliative Care, our aim was to highlight the contribution of each palliative care team member, the collaboration of the interdisciplinary team (IDT), and the patient care conference discussions.

Methods: We partnered with internal informatics specialists and Cerner experts to design and implement a customized palliative care meeting note and care summary in our EHR. We used existing features within Cerner to populate important information into a summary page from various EHR locations (Mpage). The palliative care-specific Mpage contains four folders including the patient's problems list, code status, clinical notes, and the new palliative care IDT meeting note. This note documents the patient care conference and summarizes the IDT members' most recent palliative care assessments, the patient's overall inpatient plan of care, the IDT's discussion and recommendations.

Results: The palliative care Mpage and IDT meeting note have improved communication among the interdisciplinary team members and have increased visibility and accessibility of the palliative plan of care to other clinicians. These EHR documents support compliance with the required elements for quality palliative care certification.

Conclusion: We developed a sustainable tool to condense and disseminate palliative care information via the EHR. By capturing and integrating timely information from the EHR, the palliative care Mpage and IDT meeting note foster collaboration and communication.

\section{Teaching Internal Medicine Residents Using Online Module} Moses Cone Internal Medicine Residency Program

Greensboro, NC

Team/Contact Information:

Rushil V. Patel

Resident Physician

Moses Cone Internal Medicine Residency Program

1200 North Elm Street

Greensboro, NC 27401

(336) 832-7272

rushil.patel@conehealth.com

Project Team Members:

Elizabeth Golding, DO - Director, Cone Health

Aaron Lampkin, MD - Cone Health

Mary Larach, Nurse Practitioner - Cone Health

Lawrence Klima, MD - Director, Moses Cone

Topic: Leveraging technology (e.g., EMR, telehealth)

\section{Description:}

Inpatient providers are sometimes challenged with advanced care planning for their patients. At a teaching hospital, resident physicians find themselves tasked with this responsibility for inpatients they may have only just met, as well as in their assigned 
outpatient panel. Education on communication skills though has been shown to improve resident comfort level and knowledge of discussing goals of care and advance care planning. Methods: Internal medicine categorical residents $(n=15)$ and preliminary $(\mathrm{n}=2)$ residents at a university-affiliated, community hospital learned the fundamentals of discussing goals of care and advance care planning using selected modules from the Center to Advance Palliative Care's (CAPC) online curriculum in communication skills during their outpatient clinic rotation from February through June 2016. Residents then discussed a patient encounter from their experience with their colleagues along with a member from the inpatient palliative care team and a clinical psychologist in weekly 1-hour afternoon sessions. A pre- and post-intervention survey was administered and recoded by a numerical identifier to assess the change in each resident's comfort level and knowledge level with conducting these discussions with their patients. Sessions were also audio recorded to identify recurrent themes and insights for future follow-up projects. Results: Final analysis $(\mathrm{n}=11)$ demonstrated a statistically significant improvement in the median difference between overall resident comfort level $(\mathrm{p}=0.017)$ and knowledge $(\mathrm{p}=0.014)$ of discussing goals of care and advance care planning. Several participants also expressed desires for additional opportunities to practice these skills under supervision by experienced providers. Conclusions: Residents value developing skills requisite to facilitate goals of care discussions and advance care planning. Further opportunities for practice and feedback should be identified. Reused with permission from the American Society of Clinical Oncology (ASCO). This abstract was accepted and will be presented at the 2016 Palliative Care in Oncology Symposium. All rights reserved.

\author{
49. Systems Integration of Palliative Care into Nursing \\ Homes \\ New York Presbyterian Queens \\ Flushing, NY \\ Team/Contact Information: \\ Cynthia X. Pan, MD \\ Chief, Division of Geriatrics and Palliative Care Medicine \\ New York Presbyterian Queens \\ 56-45 Main Street \\ Flushing, NY 11355 \\ (718) 670-2434 \\ cxp9001@nyp.org
}

Project Team Members:

Caroline Keane, RN, MSN, ANP, CCM

Sarah Kalinowski

Topic: Integration of palliative care to specific settings (e.g., hospital, ICU, home, nursing home, clinic)

Description:

Background: The New York State Delivery System Reform Incentive Payment Program (NYS DSRIP) aims to reduce avoidable hospitalizations and emergency department (ED) visits for Medicaid Beneficiaries. This initiative aims at reducing avoidable admissions by integrating primary palliative care $(\mathrm{PC})$ practices into nursing homes $(\mathrm{NH})$, clarifying goals of care and options, addressing pain and other symptoms, thus patients may require less hospital readmissions.

Objectives: In the long term:

1) Integrate $\mathrm{PC}$ into practice model of participating $\mathrm{NHs}$;

2) Provide education to patient-family-caregivers on PC services and their importance in healthcare;

3) Reduce avoidable readmissions/ED visits for Medicaid beneficiaries. Methods: Systems-level interven- tion initiated by New York Presbyterian Queens hospital (NYP/Q) to engage and collaborate with community $\mathrm{NHs}$ to provide primary $\mathrm{PC}$ services to seriously ill residents in NHs.

The NYP/Q Performing Provider System (PPS) recruited NHs and hospice agencies to participate. Project is co-chaired by director for transitions of care/case management and PC physician/leader, with strong administrative support. The PPS contracted or developed partnerships with community resources, including Hospices, to bring PC supports-services into NHs. PPS engaged NH staff in trainings to increase roleappropriate competence and comfort in PC skills.

To provide primary PC education, the PPS team used AMA's Education on Palliative and End-of-life Care (EPEC) training modules, providing CME-CEU accreditation for $\mathrm{NH}$ providers. EPEC covers 16 modules, including legal/ethical aspects of care, pain management, goals of care, advance care planning, and others. PPS promoted use of advance directives tools, eg, Medical Orders for Life-Sustaining Treatments (MOLST). PPS aimed to identify and engage $\mathrm{PC}$ champions at each $\mathrm{NH}$ and hospice agency.

Results: Since April 2015, NYP/Q PPS has engaged 27 NHs and 6 hospice agencies to participate. The PPS is working with partners to provide education on primary PC skills to staff, monitor clinical quality metrics associated with pain management and advance directives, and engage patients and caregivers in PC services. In Year 1 (4/1/2015 - 3/31/2016), PPS engaged 1,076 patients with 22 of the NH partners. In Year 2 to date (4/1/2016 - 6/30/2016), PPS engaged 598 patients with 20 of the NH partners. EPEC training began in Feb 2016, offered 2 modules at a time, with different NHs volunteering to host the training. Six modules have been taught so far, with positive feedback and attendance. Many NH physicians feel uncomfortable conducting basic PC conversations such as disclosing bad news, discussing goals of care, or end-of-life care.

Conclusion: DSRIP is an innovative NYS initiative to improve care and reduce avoidable hospitalizations and ED visits. NYP/Q PPS was able to form a coalition of NHs and hospice agencies, and engage patients in PC. It is too early to assess longterm outcomes such as avoidable readmissions. Next steps: Identify and engage PC champions at each $\mathrm{NH}$ and hospice agency. Pilot PCOS (Palliative Care Outcome Scale) tool at a partner NH, with goal of rolling out to all $27 \mathrm{NHs}$. Provide supportive basic education to $\mathrm{NH}$ physicians.

50. Effect of a Residential Dog on Quality of Life and Loneliness in Veterans Living at the North Florida/South Georgia VAMC, Community Living Center Lake City Division

NF/SG VAMC

Lake City, FL

Team/Contact Information:

Ellen E. Boehmer, MSN

Clinical Nurse Educator, Hospice

NF/SG VAMC

619 S Marion Ave.

Lake City FL 32025

(386) 755-3016 x3002

ellen.boehmer@va.gov

Topic: Measuring impact and value Description:

As our Veteran population ages many will come to live on our Hospice neighborhood. Several studies demonstrate that animals enhance and enrich quality of life. Little research has 
been done in the area or the effect of a dog residing on Hospice. This two-phase study was to examine the effects of having a residential pet (Jodi) on the Hospice neighborhood at the Lake City Division on NF/SG VHS. Phase I was to use qualitative interviews with Veteran residents to determine the impact of having a residential tog the Hospice neighborhood, Phase II was to measure the effects of the residential dog on self-reported loneliness in Hospice residents without cognitive impairment. Methods included the UCLA Loneliness Scale and a questionnaire reporting frequency of visits with Jodi, extent of contact with her, number of visits weekly and self-reported "feelings they had before and after contact with her. The study was stopped and closed due to three main reasons:

Low numbers of subjects and inability to reach statistical power and time barriers encountered by the primary investigator. It is the PI's hope that another facility with a residential dog on Hospice will see the value of continuing this study.

\section{Surveying an Advanced Ill Population}

North Shore University Hospital at Northwell Health

Manhasset, NY

Team/Contact Information:

Tara Liberman, DO

Associate Chief

Northwell Health

300 Community Drive

Manhasset, $N Y 11030$

(917) 837-2030

tliberma@nshs.edu

Project Team Members:

Tara Liberman, DO

Mary Curtis, PhD

Kathleen Cascio

Nicole Benincasa

Topic: Measuring impact and value

Description:

Background: Measuring patient satisfaction is increasingly a healthcare priority. HCAHPS scores are the most frequently used tool to measure patient satisfaction regarding their hospital stay. Unfortunately, these national surveys do not reflect outcomes specific to palliative care (PC), or provide feedback specific to the consulting team or any feedback from deceased patients. Our goal is to develop a feedback tool and demonstrate its utility for providing feedback from patients and families receiving hospitalbased palliative care.

Methods: We developed a brief questionnaire that focuses on symptom control, communication, advance care planning, and assistance with cultural, religious or spiritual concerns. Surveys were conducted at North Shore University Hospital of Northwell Health. In an effort to limit bias, surveys were conducted by volunteers and answers were recorded on iPads via Survey Monkey. Outcomes were immediately reported to the PC team and reviewed to improve care.

Results: The 8 item questionnaire was administered to 53 respondents from the Palliative Care Unit (PCU) and 54 respondents from the Consult Service from August 2015 through June 2016. The tool had previously been piloted in paper-based for with patients at Long Island Jewish Medical Center. Patients and/or families answered the questions on a scale from 1 to 5 , with 5 being the highest score. Both the PCU and the Consult Service achieved an overall score of 4.7 , with neither service receiving below a 4.4 on any question. Any complaints or need for symptom control was responded to in a timely manner, usually within 24 hours.
Conclusion: The process and results of these preliminary questionnaires are encouraging. Results highlight the areas where improvements can be made. Initial utilization already created changes in our service where we identify the team with pictures so the patient and family is clear as to whom we are speaking about. We continue to administer and improve the questionnaire for future patients. Our next steps can include replicating the process at a similar hospital in our health system, focusing on earlier reporting of needed symptom management and improving ways of documenting goals of care conversations. This process appears to be easily utilized and replicable across other hospitals.

\section{Study of ICU Patients Transferred to a Palliative Care Unit}

North Shore University Hospital at Northwell Health

Manhasset, NY

Team/Contact Information:

Amelia Conner

North Shore University Hospital at Northwell Health

300 Community Drive

Manhasset, $N Y 11030$

(917) 837-2030

afc5bc@virginia.edu

Project Team Members:

Amelia F. Conner

Bridget Earle, $M D$

Danielle Turrin, DO

Topic: Quality improvement

Description:

Introduction: North Shore University Hospital (NSUH) has a 10-bed Palliative Care Unit (PCU) that admits over 500 patients annually for symptom management. $25 \%$ of these patients come from an Intensive Care Unit (ICU). The unit was designed to implement palliative symptom relief and hospice care for end of life patients including mechanically ventilated patients. Literature demonstrates the role of palliative care as an essential component of comprehensive medical care. In order to provide high quality care to ICU patient transfers, we wanted to better understand the intricacies of their hospital course.

Method: A retrospective review was conducted for PCU transfers from April 1, 2016 - June 30, 2016 and specifically ICU transfers. All data was collected from Electronic Medical Records (EMRs). This data included patient demographics, hospital units, hospital length of stay (LOS), PCU LOS, admission to PCU consult time, and PCU consult to PCU admission time.

Results: From April 1, 2016 - June 30, 2016, 130 patients were admitted to the PCU. Of these patients, 30 came from ICUs. For all 130 patients, the average hospital LOS ranged from 11.215.4 days with an average PCU LOS ranging from 3.7-5.2 days. For ICU patients, hospital LOS range from 12.4-18.0 days while PCU LOS stay was 2.5-5.0 days. The average admission to PCU consult time for all patients was 4.4 days while for ICU patients it was 7.8 days. $61.5 \%$ of all PCU patients expired in the unit during this time period, $92.3 \%$ of the ICU patients expired.

53. Part-Time Fellowship Training in Palliative Medicine North Shore University Hospital, Northwell Health System Manhasset, NY

Team/Contact Information:

Sindee Weiss-Domis, MD

Assistant Professor of Medicine

North Shore University Hospital, Northwell Health System 
300 Community Drive

Manhasset, NY 11030

(516) 562-8825

sweiss3@northwell.edu

Topic: Palliative care education

\section{Description:}

North Shore University Hospital, member of the Northwell Health System, has a growing Hospice and Palliative Medicine Fellowship training program. Established in 2007 and accredited in 2009 the program spans three major hospitals and is currently approved for eight fellows.

As the number of people with serious medical illness increases, the need for hospice and palliative medicine trained physicians increases in parallel. Additionally, providers can no longer sit for the certification exam using verification of clinical experience. There are providers who wish to complete fellowship training but face hardships, financial or otherwise, presenting a barrier to pursuing a full-time program.

Changes in the ACGME program requirements for July 2015 follow; Int.C. A fellowship program in hospice and palliative medicine must consist of 12 months of education in the subspecialty. (Core) ${ }^{* 1}$ Review of the accompanying FAQs shows that the RRC allows HPM fellowship programs the flexibility to accept fellows with the expectation of completing the program on a part-time basis over the course of 24-36 months. ${ }^{2}$

The division of Geriatric and Palliative Medicine at North Shore University Hospital piloted a part-time fellowship training program in hospice and palliative medicine on July 1st, 2015. The program is in its second year. Selected candidates were from physical medicine and rehabilitation, pediatric critical care and emergency medicine. Their primary boards were contacted and agreed in writing to allow entrance to the certifying exam upon successful completion of all program requirements over a 24 month period. Each candidate alternates working in their primary field with a fixed interval of fellowship training. We anticipate that all are going to successfully complete the program.

We believe this to be a viable alternative to full time training, allowing more providers the acquisition of subspecialty competencies and board certification.

\section{References}

1. https://www.acgme.org/acgmeweb/Portals/0/PFAssets/ ProgramRequirements/540_hospice_and_palliative_medicine_ 2016_1-YR.pdf (accessed 3/1-5/4/2015)

2. https://www.acgme.org/acgmeweb/Portals/0/PDFs/FAQ/540_ hospice_and_palliative_medicine_FAQs_07012015.pdf (accessed 3/1-5/4/2015)

\section{An Evaluation of the NiPCAS}

Cohen Children's Medical Center-Northwell Health

New Hyde Park, NY

Team/Contact Information:

Deborah A. Lawrence, $M S, R N C-O B$

Nurse Manager

Cohen Children's Medical Center-Northwell Health

269-01 7th Ave.

New Hyde Park, NY 11040

(718) 470-3381

zdalzz@aol.com

Project Team Members:

Deborah A. Lawrence, $M S, R N C-O B$

Diane Shimborske, BSN, RNC-NIC

Topic: Pediatrics
Description:

Objective: The rigor of the Neonatal Palliative Care Attitude Scale (NiPCAS), developed by Victoria Kain (2009), was evaluated to provide information about a measurement relative to the area of palliative care implementation in the Neonatal Intensive Care Unit (NICU).

Background: Infants can live for various periods of time with life-limiting conditions, and focus of hospital care can shift from rescue to palliation. The NiPCAS was developed to measure NICU nurses' attitudes about palliative care, which remains underutilized in the neonatal population.

Evaluation: After a review of the literature, the conceptual framework of diffusion of innovations was used to guide creation of a scale with demographical and attitudinal items. Face and content validity were established by an expert panel. Reliability was established through test-retest, and calculation of Cronbach's alpha (.87).

Results: Exploratory factor analysis classified scales and subscales within the measurement, establishing three factors that either promoted or hindered palliative care: organization, resources, and clinical. Facilitators and barriers to implementation of palliative care were identified. Other research projects had similar findings.

Conclusion and Implications: Small sample size of the pilot study limited verification of reliability. Additional projects, which use confirmatory factor analysis, are required, and should include other practitioners, such as physicians and nurse practitioners. Evidenced-based care guidelines that are adaptable to individual NICUs, whose settings, cultures, and practices demand systematically unique standards, are desirable, as are implementation of formal training programs and supportive measures for clinicians.

\section{Developing Supportive Oncology Program in a Cancer Center}

Northwell Health

Manhasset, NY

Team/Contact Information:

Diana C. Martins-Welch, MD

Attending Physician

Northwell Health

300 Community Drive

Manhasset, NY 11030

(646) 279-0501

dmartins1@northwell.edu

Project Team Members:

Diana Martins-Welch, MD

James D'Olimpio, MD

Tara Liberman, DO

Maria Carney, $M D$

Topic: Integration of palliative care to specific settings (e.g., hospital, ICU, home, nursing home, clinic)

Description:

Background: Cancer patients experience significant symptom and psychosocial burden (1). Integration of supportive oncology programs within cancer centers has developed since the findings of Temel and Jackson. Addressing a growing need to manage patients comprehensively, the Northwell Health Divisions of Geriatric and Palliative Medicine and Hematology and Oncology partnered to initiate a community-based supportive oncology program. A needs assessment was completed of the providers, staff, and important stakeholders.

Objective: To assess the oncology staff's needs in serving their patients to affect the design of a community-based Supportive Oncology program. 
Methods: A sixteen-question survey was created and distributed to the staff at the cancer center. Questions focused on providers' need for support in fifteen areas. The target audience included physicians, fellows, mid-level providers, registered nurses, social workers, dietitians, and care navigators. A student intern administered surveys over a six-week period.

Results: A total of 61 surveys were collected. Of these, 30 were completed by registered nurses, 13 by physicians, 9 by mid-level practitioners, and 3 by social workers. Physicians expressed the highest level of need $(>4.5 / 5)$ related to nutritional status, psychosocial factors and pharmaceutical review. Mid-level providers expressed highest level of need related to pain and depression. Nurses expressed highest level of need related to psychosocial factors, pain, depression, fatigue and pharmaceutical review. The average level of need across all areas and all respondents was 4.2 on a 5 point scale. The biggest challenge identified was lack of time to perform comprehensive assessments of patients.

Conclusion: A high level of need for a supportive oncology program was identified. Per our assessment, providing comprehensive care with an emphasis on symptom control, nutritional and psychosocial support, and pharmaceutical review would benefit both providers and patients. Time constraints were identified as an issue by all practitioners and staff. The survey results supported the need for an added level of multi-disciplinary support for oncologists to care for cancer patients in the community.

(1) N Engl J Med. 2010 Aug 19;363(8):733-42. doi: 10.1056/ NEJMoa1000678.

56. Using Metrics To Evaluate In-Patient Palliative Service Northwell Health/North Shore University Hospital

Manhasset, NY

Team/Contact Information:

Santiago Lopez, MD

Northwell Health

300 Community Drive

Manhasset, $N Y 11030$

(516) 562-0100

slopez12@northwell.edu

Project Team Members:

Santiago Lopez, MD

Bridget Earle, $M D$

Tara Liberman, DO

Maria Carney, MD

Regina Roofeh, BS

Topic: Measuring impact and value

Description:

Introduction: North Shore University Hospital (NSUH) is an 806-bed community teaching hospital with a robust palliative care consultation service that sees over 2,000 new consultations per year. With the establishment of the Palliative Care Information Act, the number of hospitals in the United States offering palliative care services has grown, with $67 \%$ of US hospitals with 50 or more beds having an inpatient palliative program.1 As these programs evolve, institutions such as the Center to Advance Palliative Care (CAPC) have developed strategies to help palliative care programs best utilize resources in order to grow. Among these strategies, CAPC has defined metrics for palliative care programs to define strategic data needed for important aspects such as planning, quality improvement, and allocation of resources. 2 To better understand and improve our services at NSUH we conducted a review of the operational metrics recommended by CAPC.

Method: A retrospective review was conducted of all initial consultations between January 2015 and March 2016 via elec- tronic medical records (EMR). Data collected included demographics, top 10 ICD 9 (International Classification of Diseases) diagnoses, referring physician, service/unit, disposition, and hospital length of stay (LOS).

Results: There were a total of 2,374 patients consulted. Over $79 \%$ of patients were older than 65 years old and the majority of them were white. The leading primary diagnoses were Infections (17\%), Cerebrovascular Disorders (7\%), and Congestive Heart Failure (2\%). The average days from admission to consult were 2.9 days and the average LOS was 8.6 days. $42 \%$ of consulted patients had a routine discharge, $34 \%$ expired during the admission, $8 \%$ went to inpatient rehabilitation facility, $7 \%$ to inpatient hospice, and 5\% to home hospice.

Conclusion: The palliative care consultation service at NSUH serves a vulnerable elderly population. Special interest is to be given to infections once they are also the primary diagnosis on consults called after 15 and 30 days during the hospital stay. Hematologic malignancies and encounters related to chemotherapy were the primary diagnosis on consults called 45 days after admission. Only $12 \%$ of patients were enrolled into hospice care. $66 \%$ of patients consulted survive the hospital stay. Operational metrics has helped with developing educational programs and partnerships among other specialties, particularly hospitalist, neurology, oncology, and infectious diseases in order to provide palliative care services earlier during hospital admission. This data is also helping in planning future projects to compare the impact of palliative care services on LOS. Furthermore, there is ongoing work into helping other provider to identify hospice eligible patients and reaching out for enrollment.

\section{Building Primary PC Competencies in Optum LTC APRNs \\ Optum Complex Care Management}

Eden Prairie, MN

Team/Contact Information:

Suzanne Marshalonis, RN, BSN, CHPN

Director of Clinical Operations

East Region, Optum

Optum Complex Care Management

11000 Optum Circle

Eden Prairie, MN 55344

(877) 765-4445

suzanne_marshalonis@optum.com

Project Team Members:

Suzanne Marshalonis, RN, BSN, CHPN

Nikki Davis, MSN, FNP-C, GNP-BC, ACHPN

Summer Galecki, APRN-BC

Kevin Henning, MD, FAAFP, FAAHPM

Brian Heppard, MD, CMD

Susan Mullaney, DNP, APRN, GNP-BC

Topic: Palliative care education

Description:

Objectives:

- Describe the primary palliative care competency needs for nurse practitioners in the long term care (LTC) setting

- Describe methods that can be used on a national scale to educate and mentor APRNs on primary palliative care (PC) skills

Background: The Optum Institutional Special Needs Plan (ISNP) program serves residents of LTC facilities utilizing advanced practice clinicians to deliver longitudinal care. Effective delivery of this program requires each clinician to be 
proficient in primary $\mathrm{PC}$ competencies including conducting advance care planning conversations, identifying goals of care, and providing appropriate symptom management. The purpose of the advanced illness program is to provide ongoing education, mentoring, and national oversight in building primary PC competencies for clinicians within the organization.

In early 2015, there was an increase in acute hospital utilization rates in the ISNP program. An internal review of hospitalizations in Q1 revealed that 26\% of those hospitalizations occurred in patients who were identified as having primarily comfort goals of care. As a result, an intensive education effort was undertaken to focus on building the primary PC skills of the Optum clinicians.

Methods: Two approaches provided the intensive focus on the advanced illness education program in 2015. For states with a high number of new APRNs or APRNs that lacked PC experience, a one day in-person training program was conducted to teach primary PC skills.

In states with more senior staff and an established "advanced illness team," a train-the-trainer approach was utilized focusing on contingency planning and symptom management.

Results: Three hundred fifty clinicians across seven states were trained as part of the one day education program. They completed surveys at the completion of the training which indicated that $341(97.42 \%)$ felt that the program helped build their advance care planning skills and 290 (83.14\%) strongly agreed with the statement "The exercises contributed to my learning and helped develop my ACP skills." Additionally, 345 $(98.57 \%)$ of the clinicians indicated the training was useful to their practice and $309(88.29 \%)$ strongly agreed with the statement "I will use this information in daily practice."

The hospital admits/1000 improved throughout the year to a final year total of 262 admits/1000 for the ISNP program. An internal review of the hospitalizations indicated that the number of hospitalizations for comfort focused goals of care patients decreased from $26 \%$ in Q1 to a year-end total of $18 \%$.

Conclusions: Focusing on training and ongoing support for building primary PC skills in advanced practice clinicians impacts clinician confidence in conducting advance care planning conversations and can reduce unnecessary hospitalizations in patients who desire a comfort focused plan of care.

Implications: Building primary PC skills in advanced practice clinicians needs to be an ongoing process utilizing a variety of methods. Further outcomes/measures from the education program and model could be explored to gain visibility into the impact on patient care, patient satisfaction, and clinical outcomes.

\section{Pharmacy Services in an Outpatient Palliative Care Clinic}

Providence St. Peter Hospital

Olympia, WA

Team/Contact Information:

Ryan P. Nottingham, PharmD

Providence St. Peter Hospital

413 Lilly Road NE

Olympia, WA 98506

(360) 486-6705

ryan.nottingham@providence.org

Project Team Members:

Gregg VandeKieft, $M D, M A$

Kevin Murphy, MD, MSW

Topic: Quality improvement

Description:

Purpose: Palliative care patients require significant pharmacologic intervention. Pharmacy services within palliative care is a developing field that may contain insight into optimal medication management. Patient quality of life is a central focus and can be influenced by symptoms, side effects, and pill burden. This IRBdesignated quality improvement project was designed to measure patient, family, and staff satisfaction with pharmacy services provided in an outpatient palliative care clinic. In addition, pharmacy-driven interventions were evaluated and palliative care staff satisfaction with student involvement was assessed.

Methods: Pharmacy services were integrated in a new outpatient palliative care clinic in which a pharmacist and student pharmacist saw patients together with other members of the interdisciplinary team. Three Likert scale surveys were drafted to assess patient, family, and palliative care staff satisfaction with pharmacy services. A patient and family survey assessed pharmacy services on: positive interaction with patient/family, productivity, listening ability, ability to direct therapy, and patient's willingness to see pharmacy again. The first palliative care staff survey assessed pharmacy services on: productiveness, contributions, preparedness, effectiveness, bedside manner, improvement in patient care, improvement in medication management, and influence in patient care. The second staff survey examined the student pharmacist's role with regards to: benefit to the patient, team, and student, experience uniqueness, educational experience, willingness to work with future students, and staff support for a future palliative care rotation. Pharmacy interventions were tallied after each patient visit.

Results: In the 3-month survey period, twenty-five patients (92.6\% response rate) completed the patient survey. Scores ranged from 4.32-4.67/5, with 5 being strongly agree. Five staff members (100\% response rate) completed the staff surveys. The pharmacy services scores ranged from $4.8-5 / 5$. The student involvement scores ranged from 4.6-5/5. Pharmacy interventions averaged to 0.84 interventions per patient surveyed.

Conclusion: The response to pharmacy services within the outpatient palliative care setting was markedly positive, from both the patient and healthcare worker standpoint. Healthcare professionals and patients recognize the profound influence pharmacy services can have on medication management in this specialty. There is a fine balance in palliative care to provide superior symptom relief with fewer medications. This goal may be more easily attainable with pharmacy services involvement. Palliative care offers a unique learning experience to student pharmacists that would highly benefit them and their future patients. This quality improvement project demonstrates how pharmacy services can be a favorable addition to an outpatient palliative care clinic. This project also demonstrates the valuable learning experience student pharmacists can obtain by having a rotation in a palliative care clinic.

\section{Caring for Our Team While Honoring Our Patients Schneck Medical Center \\ Seymour, IN}

Team/Contact Information:

Donna Butler, NP

Schneck Medical Center

411 W. Tipton Street

Seymour, IN 47274

(812) 522-2349

dbutler@schneckmed.org

Project Team Member:

Anita K. Collins, MSN, FNP-BC

Topic: Team wellness

Description:

Introduction: In our rural hospital, the outpatient palliative care clinic is embedded in the cancer center. The palliative care 
and oncology teams develop strong relationships with their patients and family. We identified increasing distress in the teams with multiple losses and limited opportunity to grieve their losses. Literature recommends development of positive health care behaviors and coping strategies to prevent burnout that can occur as a result of chronic exposure to stressors and ineffective coping resources. Our palliative team developed a two-fold service to provide care to our team members along with honoring the patients we have lost.

Methods: Two to three times a year our palliative care team coordinates a Memorial Service. The palliative care and oncology teams are invited to attend. The service provides our teams the opportunity to acknowledge and reflect on losses, to focus on team care and to participate in an interactive art project that honors the patients we lost.

Examples of memorial services:

1. Devotion led by chaplain around the book "The Fall of Freddie the Leaf" by Leo Buscaglia. A team member painted a picture of a tree with branches. As patient's names were read team members reminisced about their patient. Using an inkpad the members placed a fingerprint leaf on the tree to represent each patient. This created a beautiful tree full of multi-colored leaves.

2. Chaplain provided "Blessing of Hands" with readings, ceremonial washing of team member's hands and anointing of oil. A team member painted a picture of a meadow. As patient's name was read, team members reminisced about their patient. Using inkpad with stamps members added flowers and butterflies to represent each patient. This created a beautiful meadow full of flowers and butterflies.

Results: Satisfaction surveys were completed by the team members following the memorial service. The services have been well received. Based on recommendations we will expand the invitation to our colleagues in hospice, on the medical floor and ICU as they too care for this group of patients and experience similar loss.

\section{Quality Palliative Care: Are We Delivering Our Very} Best?

Shawnee Mission Health

Shawnee Mission, KS

Team/Contact Information:

Cindy Kuklenski, RN, BC

Pain Management/Palliative Care Nurse

Shawnee Mission Health

9100 West 74th Street

Shawnee Mission, KS 66204

(913) 676-2224

cynthia.kuklenski@shawneemission.org

Topic: Quality improvement

\section{Description:}

The National Consensus Project for Quality Palliative Care published the third edition of "Clinical Practice Guidelines for Quality Palliative Care" in 2013. The guidelines contain eight domains which identify core concepts of high quality palliative care. The guidelines serve as a tool to promote consistently high standards to new and existing palliative care teams.

In a 530 bed acute care hospital, our palliative patient census increased by 55\% between 2012 and 2015; our small, multidisciplinary team received 637 consults in 2015. Numbers in 2016 continue to escalate each month. To sustain the growing service, we acquired new team members, including new palliative care physicians and social workers.
In an effort to sustain consistently high standards, and to stimulate an environment of continuous service advancement, our team launched a three-phased quality improvement project, centered around the guidelines identified by the National Consensus Project for Quality Palliative Care.

Phase I: A tool titled: "Palliative Care Evaluation" (PCE) was developed to critique/evaluate care delivery, addressing whether or not the standards put forth in each of the eight domains were fulfilled.

Phase II: Utilizing the PCE tool, a retrospective gap analysis was conducted; the measurement was between the eight domains (as appropriate), and the electronic medical record (EMR) clinician documentation. This analysis was completed by the palliative care nurse, then reviewed with the team members.

Phase IIIa: Currently underway: team members including physician, nurse, and social worker, are completing the PCE tool after each initial patient/family meeting in "real time." The team then collaboratively reviews the results.

By reviewing each members' perspective on successes and/or challenges, the team is provided the opportunity for safe and immediate debriefing and evaluation. Plans for subsequent follow-up with patient and family ensue.

Phase IIIb: The palliative care nurse then reviews the EMR to ascertain whether the documentation reflects those standards of best-practice.

This poster will reveal the PCE tool in Phase I, disclose the results of Phase II, and give updates on the ongoing Phases IIIa and IIIlb. Patient demographics will be included.

Most importantly, this poster will discuss how, by utilizing these standards, our team is improving the way we deliver palliative care to our patients.

\section{RAIs for Use within Geriatric Care Plans for Home- Based Palliative Care}

Snowline Hospice

Diamond Springs, CA

Team/Contact Information:

Maritza M. Madrigal, MSW

Supportive Care Social Worker

Snowline Hospice

6520 Pleasant Valley Road

Diamond Springs, CA 95619

(530) 621-7820

mmadrigal@snowlinehospice.org

Topic: Equitable access to palliative care for vulnerable populations

Description:

Older at-risk adults with chronic, terminal illness would benefit from home-based Palliative Care (PC) that includes a comprehensive, holistic assessment of a patient's needs, strengths, and vulnerabilities created by a gerontologist. Currently, there is a critical need for gerontologists to be part of PC interdisciplinary teams (IDT), alongside social workers, nurses, and physicians. The goal of this culminating project was created to identify health risks of older adults receiving PC utilizing the Association for Gerontology in Higher Education (AGHE) Core Competencies as a framework. Gerontologists within PC practice can support social workers, who often lack geriatric-specific training in their educational curriculum, by creating comprehensive, holistic care plans that address individual patient goals for end-of-life treatment. Patients with a chronic disease or a terminal illness face many options regarding the care and treatment they wish to receive. A gerontologist can explore a patient's motivation and values by implementing the concepts of Transtheoretical Model 
(TTM) Stages of Change (SOC) through the use of Motivational Interviewing (MI). The conversation regarding end-of life-care would be created based upon the stage the patient is currently in (i.e., pre-contemplation, contemplation, preparation, or action) assuring it to be completely patient-centered. In subsequent meetings with patients and families, a reappraisal of those decisions would promote informed end-of-life planning and self- management of an ongoing illness. A survey administered to Snowline Hospice IDT demonstrated that $85 \%$ of participants desired increased training in common geriatric assessment tolls. This project demonstrates a new model of home-based PC delivery to at-risk older adults living in rural areas. El Dorado County is experiencing close to a $400 \%$ increase in those age 85 and older and $82 \%$ of the population live in unincorporated areas of the county and $35 \%$ are considered rural. Geriatric care plans which include rapid assessment instruments can be the catalyst for discussions regarding end-of-life planning for patients and families and provide an overall comprehensive plan for the patient and family.

62. Palliative Care at South County Hospital: System Wide South County Hospital

Wakefield, RI

Team/Contact Information:

Holly Fuscaldo, BSW

Case Manager

South County Hospital

100 Kenyon Avenue

Wakefield, RI 02879

(401) 788-1505

hfuscaldo@southcountyhealth.org

Project Team Members:

Holly Fuscaldo, BSW

Lynne Driscoll, $R N$

Topic: Health system strategies

Description:

South County Health is taking a system wide approach to managing patients with chronic disease to support symptom management and identifying goals of care. Our inpatient palliative care consulting team consists of physician and nurse practitioner from Hope Hospice and Palliative Care of Rhode Island and an interfaith chaplain and social worker employed by South County Health. The nurse practitioner follows patients that are discharged to skilled nursing facilities for continued palliative care services, assists patients and families with transition to the community, including transition to South County Home Health Homecare. The social worker is capturing patients through STAR (Survivorship training and rehabilitation) who require continue support and proactive rehabilitation with cancer patients to improve quality of life. We communicate with primary care providers and specialists involved with long term management of the disease process to enhance overall quality of life and improve transitions of care and communication.

At South County Hospital, palliative care facilitates communication between patients and caregivers, while improving the coordination of care. We advocate for patients goals of care and work with their healthcare team to meet their specific goals. Our focus is to have our patients maintain control over their care and not have their care control them. As a patient's condition and treatment options change and evolve, we assist in providing the right kind of care at the right time.

The team has developed:

- Admission screening tool for Nursing.

- Spiritual Assessment tool.
- Progress notes that captures change in advanced care planning.

- Flag in EMR that capture patients that are Palliative Care with (purple P).

- Palliative Care Registry.

Metrics

- Consults by month.

- \% of patient discharged with a MOLST form.

- \%of spiritual care assessments.

- Readmission post palliative care consult.

- Days from admission to consult.

- \% of patients that score $3,4,5$ or greater that did not receive consult.

- Consults by providers.

- Top DRG's.

63. Gaps in Adult End of Life Education: A Multimodal Approach

Stanford Health Care

Stanford, CA

Team/Contact Information:

Karla Schroeder, DNP, MHA, ANP-BC

Director of Palliative Medicine and Geriatrics

Stanford Health Care

300 Pasteur Drive

Stanford, CA 94305

(650) 723-1303

kschroeder@stanfordhealthcare.org

Project Team Members:

Karla Schroeder, DNP, MHA, ANP-BC

Judy Passaglia, CNS, RN, ACHPN

Topic: Palliative care education

Description:

Nursing plays a crucial role in the improvement of end-oflife care. In an academic medical center effort must be made to prepare nursing staff to care for a diverse patient population, high acuity, psychosocial factors, intense family dynamics and the emotional response to death. It is an important part of nursing wellness and resilience to anticipate personal reactions and develop appropriate coping mechanisms when providing end-of-life care. This dynamic requires an innovative educational plan which addresses the intensity of the patient population as well as the organizational and nursing culture. Many challenges exist to ensure that dying individuals receive competent and compassionate care as they face the end-of-life. To meet these challenges, we have developed a teaching program to support the nursing staff in obtaining these skills.

The initial group consisted of fifty registered nurses from one medical surgical unit. This nursing unit cares for the general inpatient hospice patients and has the highest number of end-oflife "comfort care" patients in the organization. The instructional model for end-of-life education starts with pre-education Health stream learning modules on palliative medicine, pain management, symptom management and end-of-life care. These foundational computer modules then continue into an engaging didactic classroom setting which further expands on these concepts. Additionally, self-care was introduced as a topic. In the last phase, the learners progressed to an interprofessional standard patient and family simulation training consisting of APPs, MDs, SW, chaplains, and nursing staff who were able to teach and share their experiences and expertise. Topics included 
communication, medication equivalency, and safety, ethics, pain and symptom management.

The standard patient and family simulated case scenarios brought up memories and emotions for the trainees, who were overwhelmed at times by the remembrances of both peaceful and difficult deaths. The participants provided both articulate and extensive dialogue about their experiences and interactions with members of the team. The nurses had the opportunity to debrief and discuss their anxiety, fears and the witnessed pain and suffering of dying patients. Nurses recognized the cues sent and received between the nurse the standard simulated patient and family. In the post-survey nurses showed increased knowledge with this multitiered educational program. The surveys strongly suggested continued use of open discussion and standard patient simulation in further education.

Incorporating this basic end-of-life course is just the beginning to improving care for they dying. This course will help to prepare nurses at all levels to be change agents within the prevailing health care culture. There were many nurses from this initial course who showed interest in becoming end-of-life champions. Teaching in this manner breaks down barriers and allows for a safe environment for learning as well as a culture of openness, curiosity, and commitment to evidence-based care for patients at the end of life.

\section{A Novel Approach to Outpatient Palliative Care Clinic} Flow

Stanford School of Medicine / Stanford Healthcare

Stanford, CA

Team/Contact Information:

Joshua C. Fronk, DO

Clinical Assistant Professor

Stanford Health Care

300 Pasteur Drive

Stanford, CA 94305

(650) 724-0385

jfronk@stanford.edu

Project Team Members:

Heather Shaw

Karla Schroeder

Sandy Chan

Lisa Gustofson

Kavitha Ramchandran

Gary Bertuccelli

Jeong Min Jung

Elizabeth Boatwright

Gayle Humphrey

Topic: Staffing models

\section{Description:}

Hospitals and healthcare organizations are embracing outpatient palliative medicine to complement and expand upon the work that is often started during an acute care palliative care consultation. Earlier palliative care consultation and integration in the outpatient setting can decrease hospital cost expenditures and improve patient experience and overall quality of life (2014 Institute of Medicine [IOM] Report, Dying in America: Improving Quality and Honoring Individual Preferences Near the End of Life. Key findings regarding financing healthcare near the end of life, National Consensus Project [NCP] for Quality Palliative Care [2013]. Clinical practice guidelines for quality palliative care, 3rd edition. Accessed March 6, 2016 from http://www.nationalconsensusproject.org/ NCP_Clinical_Practice_Guidelines_3rd_Edition.pdf). Different models of care are currently being utilized, including clinicbased and home-based models.
Similar to palliative care in the hospice or inpatient setting, the use of a multi-disciplinary team in the outpatient setting is a crucial component in allowing for full assessment and management of the four corners of wellness - physical, social, psychological and spiritual. The practice of palliative care is fundamentally dependent on the multidisciplinary team; however, as outpatient palliative care clinics continue to grow, there can be a struggle to maintain the multi-disciplinary model for various reasons including the mismatch between the traditional clinical template, needs of a palliative care patient and the multidisciplinary team itself. Recent research indicates that quick, minimal-style palliative care consults can actually cause more harm to patient good, indicating that developing relationships over time and leveraging the MDT is crucial to our work (Carson SS, Cox CE, Wallenstein S, et al. Effect of Palliative Care-Led Meetings for Families of Patients with Chronic Critical Illness: A Randomized Clinical Trial. JAMA. 2016;316[1]:51-62). As outpatient palliative care becomes more integrated and accepted in routine patient care, the demands of volume and time can make it challenging to incorporate the multidisciplinary team, creating the temptation to abandon the multidisciplinary team and the four corners of wellness. We will share our own challenges and successes since establishing a 5-day per week ambulatory palliative care clinic in Stanford Cancer Center, a tertiary academic medical center. As our patient volume has grown, we have implemented a unique approach to scheduling patients which has allowed for us to maintain a full multidisciplinary approach, including LCSW, chaplain, nursing and physician. Since implementation, we have been able to meet patient demand, decrease wait time from initial consultation, improve clinic flow and enhance team wellness and job satisfaction.

\section{Embedded Palliative Care in an Academic Medical Center}

The University Of Kansas Health Systems

Westwood, KS

Team/Contact Information:

Amy Velasquez, RN

Palliative Care Nurse Navigator

The University Of Kansas Health Systems

2330 Shawnee Mission Parkway

Westwood, KS 66205

(913) 588-5720

luv2runamy@yahoo.com

Project Team Members:

Wendy Thomas, RN, MSN, OCN

Karin Porter-Williamson, MD, FAAHPM

Christian Sinclair, MD, FAAHPM

Topic: Integration of palliative care to specific settings (e.g., hospital, ICU, home, nursing home, clinic)

\section{Description:}

Cancer patients and their caregivers are often burdened by multiple medical appointments each month. Although they may be receptive to the type of added support provided by outpatient palliative care providers, the addition of another appointment is too overwhelming. When forced with spreading limited resources of transportation costs, child care and missed work, patients often choose to forego outpatient palliative care appointments.

In our cancer center at the University of Kansas Health System, the Outpatient Palliative Care practice explored if seeing patients during other scheduled appointments would effectively reduce the barriers to patients taking advantage of their services. Working with the cancer center administration, our practice proposed coordinating and embedding palliative 
care visits with medical oncologist visits and treatment appointments. Our goal was to increase patients' willingness to schedule palliative care appointments, and decrease no-show appointment rates, while reducing the schedule strain on patients and caregivers.

Over the three year period of our outpatient palliative care program, we have found patients much more willing to schedule appointments that are coordinated with other cancer center visits. The no-show rate among embedded appointments was minimized with patient and staff education. In addition to achieving our original goal, we have discovered several additional benefits to embedded palliative care appointments. Providers and patients have benefited from shared knowledge, improved communication and trust building, resulting in more effective therapeutic relationships for all parties.

\section{Rounding Third: Bringing Pediatric Palliative Care Home}

Transitions LifeCare

Raleigh, NC

Team/Contact Information:

Lily Gillmor, BSN, RN, CHPPN

Pediatric Program Manager

Transitions LifeCare

250 Hospice Circle

Raleigh, NC 27607

(919) 215-9391

lgillmor@transitionslifecare.org

Project Team Members:

Lily Gillmor, BSN, RN, CHPPN

Laura Patel, MD

Christopher Thompson, MD

Topic: Pediatrics

Description:

Despite a rise nationally in the number of children living with life limiting and life threatening illnesses, the availability and quality of community based pediatric palliative care varies widely state by state. A contributing factor to this variation is the Affordable Care Act's concurrent care mandate. A few states have created successful community based pediatric palliative care programs using concurrent care, however the majority of states have done the bare minimum in offering these services. North Carolina is a state that has significantly limited community based pediatric palliative care services. With the introduction of concurrent care there was hope that there would be an increase in both the development and utilization of community based pediatric palliative care services. Unfortunately in the 6 years since the ACA, NC has made it difficult to utilize concurrent care and the lack of services has continued. Raleigh, NC has three level 1 children's hospitals within a 30 mile radius (two with inpatient pediatric palliative care teams), but until 2015 had no community based pediatric palliative or hospice care programs. Recognizing this gap in care, in the fall of 2015 Transitions LifeCare (a Raleigh, NC based non-profit company offering adult palliative and hospice care services) created a community based pediatric palliative and hospice care program, Transitions Kids. A model of care was created that provides comprehensive community based supportive care to both pediatric patients and their families. This poster describes the evolution of the model, the role of each team member, outcomes of the program in the first year, and issues surrounding financial sustainability. With a national shortage of community based pediatric palliative care programs our hope is this model can serve as a guide to creating and sustaining a program through deliberate planning and philanthropy.

67. Utilization of EHR to Support Joint Commission Advance Palliative Certification Performance Measures

Trinitas Regional Medical Center

Elizabeth, NJ

Team/Contact Information:

Geraldine Cruz, MSN, CHPN, CCRN

Director of Palliative Care Department

Trinitas Regional Medical Center

225 Williamson Street

Elizabeth, NJ 07202

(908) 994-5851

gcruz@trinitas.org

Project Team Members:

Geraldine Cruz, MSN, CHPN, CCRN

Clare Cruz, MSN, RN, BC

Hana Baudendistel, MS, BSN, NE-BC

Topic: Leveraging technology (e.g., EMR, telehealth) Description:

Trinitas Regional Medical Center (TRMC) received the initial Joint Commission Certification in Advanced Palliative Care in 2013 and redesignation in 2015. As a Joint Commission certified program, we are held to a set of standards and is required to collect and analyze data. The performance measure selected have to be evidence-based, relevant valid and reliable (www.jointcommission.org).

The Palliative Care Team collaborated with the Informatics Nurses of TRMC to explore our EHR in designing clinical documentation that will support the Palliative Care (PC) performance measures.

There are four performance measures that the program has to monitor.

1. Documentation of family meetings

2. Documented decrease in pain score

3. Improvement in the dyspnea assessment of patients, and

4. Patient satisfaction-this measure was excluded in this EHR project. Patient satisfaction is measured by evaluating the survey responses received from discharged patient who had PC consult

A family meeting template was designed as a structured note that meets the needs of PC team. TRMC adapted two evidencebased tools; (1) The Adult Non-Verbal Pain Scale (ANVPS), and (2) the Respiratory Distress Observation Scale RDOS (Campbell \& Walch, 2010). The clinical documentation in the EHR was modified and designed to accommodate these clinical assessment tools.

On July 2013, the documentation of family meeting in the EHR was piloted in the intensive care unit (ICU). The family meeting template was initially used by the PC Nurse, and eventually expanded to the medical residents, physicians, and the ICU nursing staff. The project rolled out to the oncology and geriatric unit first, then to the rest of the medical surgical units. Our data showed the progressive increase in the documentation of family meetings from our inception to present time. Our baseline data was $20 \% \%$ in 2009 . After the implementation, the initial documentation rate was 43\%, 3rd quarter 2013 .

TRMC adapted an evidence-based tool to assess respiratory distress for patients who are unable to self-report. On August 2013, RDOS was first implemented in ICU. RDOS enables the 
clinician to assess and document patient's respiratory distress using a standardized tool. Review of documentation enables us to analyze and monitor symptom improvement.

Ongoing monitoring of pain assessment and documentation is a hospital wide performance improvement activity. TRMC adapted the ANVPS and retired the previous scale used for nonverbal patients. On August 2013, the ANVPS was first utilized in the ICU for non-verbal incubated/sedated patients.

\section{References}

Campbell, M.L. \& Walch, J. (2010). A respiratory distress observation scale for patients unable to self-report dyspnea. Journal. Of Palliative Medicine. Vol. 13, No. 3.

Performance Measurement Requirements for Palliative Care. Retrieved on July 28, 2016 https://www.jointcommission.org/ certification/performance_measurement_requirements_pallia tive_care.aspx

\section{Outcomes of a Population Health Community-Based} Palliative Care Program

Trinity Health

Livonia, MI

Team/Contact Information:

Maria Gatto, MA

Trinity Health System Director Palliative Care

Trinity Health

20555 Victor Parkway

Livonia, MI 48152

(734) 343-1000

gattom@trinity-health.org

Project Team Members:

Lori Yosick, LISW-S, CHPCA

Terri Maxwell, PhD, APRN

Topic: Specific patient populations

Description:

Original Research Background: Improving the care of patients with serious illness is important for humanitarian and financial reasons. We present a population health communitybased palliative care pilot program providing advanced illness management (AIM) to a Med Advantage (MA) population. Predictive analytics used for patient selection and the clinical and financial outcomes are highlighted.

\section{Research Objectives}

1. Identify patients at risk for an over-medicalized death through predictive modeling

2. Evaluate the clinical and financial outcomes of a population health community-based AIM program

Methods: We built a predictive model to identify members at risk of over-medicalized death using the Medicare 5\% file for modeling and regression analysis. Clinical measures were selected based upon literature review; assessments captured key performance metrics.

Using the predicted risk of patients referred for intervention, we segmented the population into patients enrolled in the program and those not enrolled and compared admission, re-admission, ER visit rates of each group, and relative costs.

Results: 212 patients enrolled in the program; $88 \%$ over age $80.98 \%$ percent had goals of care addressed with $38 \%$ changing code status. Over $96 \%$ of patients reported satisfaction with symptoms and patient and family satisfaction with the program was very high. Preliminary cost savings demonstrated a net savings of $\$ 24,000$ per month/150 pa- tients. Hospice admission rate was $16.5 \%$; median length of stay of 52 days.

Conclusion: Care at the end of life was improved through better education, support, communication and coordination by skilled palliative care providers. Patients were admitted to hospice earlier, reported high satisfaction, and costs of care were lowered.

Implications for Research, Policy or Practice: The current health care environment incentivizes insurance companies to engage in population health interventions to provide their beneficiaries with the highest benefits while managing their costs. Palliative care organizations are in an excellent position to partner with payers to care for seriously ill patients in the community setting.

\section{Meaningful Impact: Automated Palliative Care} Scorecard

University of Colorado Anschutz Medical Campus

Aurora, CO

Team/Contact Information:

Jeanie Youngwerth, MD

Director of Palliative Care Service

University of Colorado Anschutz Medical Campus

12605 E. 16th Avenue

Aurora, CO 80045

(303) 556-2400

jean.youngwerth@ucdenver.edu

Project Team Members:

Sheri Kittelson

Read Pierce

Jeanie Youngwerth, MD

Topic: Measuring impact and value

Description:

Background: In response to poor health care quality outcomes and rising costs, health care reform triple aim has increased requirements for providers to demonstrate value to payers, partners and the public.

Objective: Electronically automating measurement of the meaningful impact of palliative care programs on clinical, operational, and financial systems over time is imperative to the success of the field and the goal of development of this automated palliative care scorecard.

Design: The scorecard was organized into a format of quality measures identified by the Measuring What Matters (MWM) project that are defined as important to the team, automatically extracted from the electronic health record (EHR), valid, and can be impacted over time.

Setting: The scorecard was initially created using University of Florida Health (UF) data, a new palliative care program, and successfully applied and implemented at University of Colorado Anschutz Medical Campus (CU), a second institution with a mature palliative care program.

Measurements: Clinical metrics are organized in the scorecard based on MWM and described in terms of the metric definition, rationale for selection, measure type (structure, process, or outcome) and whether or not this represents a direct or proxy measure.

Results/Conclusions: The process of constructing the scorecard helped identify areas within both systems for potential improvement in team structure, clinical processes, and outcomes. Additionally, by automating data extraction, the scorecard decreases costs associated with manual data entry and extraction, freeing clinical staff to care for patients and increasing the value of palliative care delivered to patients. 
70. Views of Cancer Patients and Lay Caregivers of Tablet Use

University of Illinois at Chicago College of Nursing

Chicago, IL

Team/Contact Information:

Marie L. Suarez, PhD

Project Director

University of Illinois at Chicago College of Nursing

845 South Damen Avenue

Chicago, IL 60622

(312) 996-7800

mlsuarez@uic.edu

Project Team Members:

Julie Glendenning

Jessica Perez

Michael Murray

Jacob Miller

Tim McCurry

Anayza Gill

Robert Shea

Karen Frank

Nargis Nardi

Yingwei Yao

Ezenwa Miriam

Zaijie Wang

Molokie Robert

Carrasco Jesus

Angulo Veronica

Marie L. Suarez

Diana J. Wilkie

Topic: Leveraging technology (e.g., EMR, telehealth)

Description:

Introduction: Tablet technologies offer new opportunities to bring the patient's and caregiver's voices into hospice and palliative care, but the acceptability of patients and caregivers using tablets as part of hospice care is not documented, especially for minorities. The purpose of this study was to describe tablet interface acceptability reported by adult hospice cancer patients and their lay caregivers.

Methods: From two hospices, we recruited patient/lay caregiver dyads. The demographic characteristics for 237 patients (mean age $68.3 \pm 14.2$, ranged from $20-100$ years, $49 \%$ male, $33 \%$ African American, 47\% non-Hispanic white, 17\% Hispanic, 3\% other ethnicity) and 235 caregivers (mean age 53.2 \pm 15.0 years, $28 \%$ male, $35 \%$ African American, $41 \%$ non-Hispanic white, $19 \%$ Hispanic, $6 \%$ other ethnicity) are typical except for higher minority participation than is usual for hospice studies. In the patients' homes, patients and caregivers used an Internet-enabled Samsung Tablet to complete the Computer Acceptability Scale (CAS, 0-9) after they used the tablet to complete pain and symptom questionnaires. Data were written to a secure server, extracted from the SQL (structured query language) database, and analyzed with statistical software R.

Results: Patients reported severe worst pain in the past 24 hours (7.02 \pm 2.34 on 0 - 10 scale) and moderate current pain $(4.68 \pm 2.64)$. For patients, the mean CAS score was $7.8 \pm 01.3$. CAS item analysis indicated that $48.6 \%$ of the patients thought the tablet was hard or somewhat hard to use or did not answer $(1.3 \%), 15.2 \%$ thought the touch screen was not easy to use, and $6.8 \%$ thought the programs should not be available to all hospice patients. For caregivers, the mean CAS score was $8.44 \pm 0.98$. CAS item analysis indicated that $19 \%$ of the caregivers thought the tablet was hard or somewhat hard to use, $9 \%$ thought the touch screen was not easy to use, and $4 \%$ thought the programs should not be available to all hospice patients. Older caregivers $\geq 65(8.15 \pm 0.99)$ reported statistically significantly lower acceptability scores than younger caregivers $(8.52 \pm 0.96, p=.016)$.

Conclusions: Patients' worst pain was higher than desirable. Patients were older than caregivers and had more difficulty using the tablet than caregivers, but both groups reported high acceptability of the tablet-based data collection process. The findings, however, point to the opportunity for improving the functionality of the tablet-based application to improve its acceptability. Additional usability research is warranted to improve the Android-based user interface for older adults. The overwhelmingly positive results support adoption of tablet technology in hospice care to improve cancer pain and symptom management.

71. An EMR Pain Page That Works: Partnering with a National EMR Vendor

University of Missouri-Tiger Institute

Columbia, MO

Team/Contact Information:

Paul E. Tatum, III, MD

Program Director Hospice \& Palliative Medicine Fellowship

University of Missouri-Tiger Institute

1 Hospital Drive, M240, DC032.00

Columbia, MO 65065

(573) 999-0355

tatump@health.missouri.edu

Project Team Members:

Paul E Tatum, MD MSPH CMD FAAHPM AGSF

Kevin W. Craig, MD, MSPH, FAAFP

Elizabeth L Kiburz, BA, MBA, PMP

Mary M. Williamson, RN, BSN, OCN, NE-BC

Tim Green, BS, PhD

Hannah M. Tomlinson

Kasie D. Sly, BS

Topic: Leveraging technology (e.g., EMR, telehealth)

Description:

Outline:

I. Obstacle: Necessary patient events that support pain management are not in a consolidated view and require additional time to dig through the chart

Background:

a) Palliative Care team Pre-Rounding

b) Tiger Institute partnership, Established in 2009 between MU Health Care and Cerner Corporation

c) Tiger Institute development team Innovation at the Edge partnered with MU Health Care Palliative Care providers to design a Pain Management Summary to aid in effective and efficient patient pain management 1.1. Description of page: Twelve-hour view of graphed pain events and vitals vertically aligned with full pain medication details table and pain events table

\section{Study:}

a) Evaluating the four primary data elements that support pain management (Pain trend over 24 hours, pain medication over 24 hours, Stool, and Respiratory Rate)

b) Time in Motion comparison between EMR standard tools and Pain Management Summary Page

c) Task Load Index comparison between EMR standard tools and Pain Management Summary Page 
d) Participants:1.1. 16 clinicians (attending physician, resident physician, medical student, registered nurse) evaluating 3 patients ( 6 total patients used)

Results:

a) Total reduction in time to find primary pain data elements: - 54\%

b) Reduction in time to find individual pain data elements:

1.1. Pain medications administered in past 24 hours: $-65 \%$

1.2. Last stool: $-55 \%$

1.3. Respiratory rate: $-43 \%$

1.4. Pain score pattern for past 24 hours: $-51 \%$ 72. Barriers to End of Life Communication: How Can We
Address Them?

University of South Florida

Tampa, FL

Team/Contact Information:

Joey V. Louis, MD

Fellow- Hospice and Palliative Medicine

University of South Florida

4202 E Fowler Avenue

Tampa, FL 33620

(813) 974-2011

joeyvlouis@gmail.com

Topic: Palliative care education

\section{Description:}

Introduction: A substantial number of patients admitted to the hospital are suffering from a terminal illness. In the teaching hospitals, care is usually coordinated by residents. We often see confusion among resident physicians, patients and family members and the lack of a clear plan for the end-of-life. As a result, communication regarding end-of-life care can be suboptimal. In our institution, we decided to identify barriers to communication around end-of-life care in order to devise a method to overcome them.

Methods: We devised a questionnaire consisting of 9 questions we encountered during our experience as residents when communicating with families and patients regarding end-of-life care decisions. Residents and house staff from the departments of Surgery, Internal Medicine and Family Medicine were asked to do the survey online or on a paper document over one month period. We also incorporated a question on which strategy they think will be useful to overcome these barriers.

Results: A total of 70 residents and house staff participated in the study. MS excel was used to collate the data and descriptive statistics was used to analyze it. Some barriers were identified. Fifty-seven percent of the residents indicated that they need more conviction when they suggest end-of-life options to patients. Forty-two percent of the residents also felt afraid to suggest a palliative care consult instead of a life-prolonging treatment. Slightly more than half of the residents $(58 \%)$ would like a training workshop where they would role-play and practice to improve their end-of-life communication skills.

Conclusion: Our questionnaire suggested that the common barriers to end-of-life communication for residents were: lack of confidence and fear to discuss end-of-life care. The proposed next step based on the survey would be to conduct and assess the effectiveness of a communication training workshop, suggested by the participants.
73. No Disease, Chronic Pain, High Dose Opioids ... What Do We Do?

Virginia Commonwealth University Health System

Richmond, VA

Team/Contact Information:

Wendy E. Thompson, NP

Virginia Commonwealth University Health System

1250 E Marshall Street

Richmond, VA 23284

(804) 914-0074

wendyedmiston@yahoo.com

Project Team Members:

Wendy Thompson, NP

Hope Barner, RN

Jessica Tsukanov, DO

Egidio Del Fabbro, MD

Topic: Specific patient populations

Description:

Introduction: Outpatient palliative care clinics are often expected to manage a broad spectrum of oncology patients. Oncology patients who have completed disease -specific treatment and no longer have any evidence of disease, present a particular challenge to the palliative care practitioner, especially with regard to their chronic opioid management. Typically, neuropathic pain related to surgery or chemotherapy is their predominant symptom complaint. The neuropathic pain experienced by patients may also be complicated by preexisting chronic pain syndromes and a history of 'chemical coping'.

Simply transitioning these patients to their primary care provider (PCP) or a chronic pain specialist may not be possible for a variety of factors. PCP's may be reluctant to assume the responsibility of chronic pain management because they lack training ; are concerned about the medico-legal risks of prescribing opioids; or have time constraints. Our institution has additional challenges, including the absence of a robust chronic pain service, and community based pain clinics that focus on providing only interventional pain procedures. There are other social and financial barriers preventing our patients from obtaining access to the appropriate care. Many are un-insured or indigent, lack transportation or have a history of substance misuse. We describe a case series of oncology patients managed in our ambulatory care clinic for chronic pain, with no evidence of disease, and complex psychosocial issues.

Methods: A case series of 7 oncology patients (4 female, 3 male Caucasians), mean age 35 years, with no evidence of disease $\geq 12$ months. Edmonton Symptom Assessment System (ESAS) scores at initial visit and last visit, morphine equivalent dose were analyzed.

Results: Six patients unemployed, one employed without insurance coverage. Their chronic pain was described as arthralgia, odynophagia, myalgia, or neuropathic pain. All 7 patients had complex psychosocial issues including poor family support, marital or child custody problems, poor financial resources, or the recent death of spouse. All had documented depression and/or anxiety. Four of the seven had past and/or present polysubstance abuse. Initial Morphine Equivalent Daily Dose (MEDD) ranged $60 \mathrm{mg}$ to $1200 \mathrm{mg}$, average $552 \mathrm{mg}$. ESAS pain scores ranged 4 to 8/ 10 (average 6/10). An individualized plan was initiated for every patient and included access to an interdisciplinary team (psychologist, physical therapist, registered nurse, physician and nurse practitioner).By their last visit, patients' MEDD range was 0 to $1035 \mathrm{mg}$, median of $25 \mathrm{mg}$. Pain ranged 0 to $8 / 10$, average $4 / 10$. Four of the 7 were able to stop taking opioids with pain scores of 
$0 / 10$. The other 3 had reductions. Average time between first and last visit was 4.14 months.

Conclusions: Oncology patients with no evidence of disease may be on high dose opioids, express high symptom burden, and experience a number of complex psychosocial issues. Although it is debatable whether the palliative care clinic is the optimal site for managing these patients, our case series shows that an interdisciplinary approach can successfully decrease opioid doses, and reduce pain even in those patients with a history of substance abuse.

\section{Palliative Care Nursing Education Initiative \\ Virginia Mason Medical Center \\ Seattle, WA \\ Team/Contact Information: \\ Edit Shalom, BSN-BC \\ RN Educator \\ Virginia Mason Medical Center \\ 1100 9th Avenue, Mail Stop G2-EDU \\ Seattle, WA 98115 \\ (206) 341-0636 \\ edit.shalom@virginiamason.org \\ Project Team Members: \\ Deborah Kelly, MN, RN \\ Edit Shalom, BSN-BC \\ Nancy Westvang, $M S, R N$ \\ Topic: Palliative care education \\ Description:}

There is a national shortage of palliative care specialists and mounting evidence that patients who receive palliative care live longer with better quality of life. At Virginia Mason Medical Center we also were experiencing a high demand for palliative care specialists. We also found that many of the requests were for generalized palliative care, which nurses can deliver with the right knowledge and skills.

Once the need for more generalized palliative care was identified, the current knowledge of our staff was assessed using the validated tool, The Palliative Care/End-of-life Professional Caregiver Survey (EPCS).

The focus of this initiative was to enhance palliative care knowledge and practices of Virginia Mason Registered Nurses to meet the needs of the patients and their families at the point of care by their familiar care providers, nurses.

The goal of the initiative was to provide nurses with the knowledge, skills and tools in order to apply general palliative care principles in their nursing practices.

The City of Hope's End of Life Education Consortium (ELNEC) was used to train 3 cohorts of nurses. A total of 46 RNs were successfully trained

Each cohort consisted of ambulatory and inpatient RNs. Cohort one was the Cardiology cohort, nurses from inpatient telemetry units and outpatient cardiology care managers were involved. Cohort two was comprised of internal medicine outpatient clinics, inpatient medical/surgical units, and Clinical Nurse Leaders from inpatient units. The third cohort was our oncology service line including RNs from outpatient infusion, oncology clinic and inpatient oncology.

Having cohorts from across our healthcare system optimized care transitions throughout the continuum of care to help support patients with serious conditions. It also provided cohesiveness and understanding between the inpatient and outpatient RNs.
Course time spent in the classroom consisted of didactic, hands-on learning, and role playing. Two hour learning seminars were held between and after the $8 \mathrm{hr}$ learning sessions which provided follow up.

In addition to the classroom sessions, there was also a "walk in their shoes" experience with Virginia Mason's Palliative Care Team. The Ns were given an opportunity to shadow with a member of the Palliative Care Team.. This allowed the RN to see the team in action, use the skills learned in the seminar, and be provided with just in time feedback from the Palliative Care team. This experience was monumental to the RNs in and their practice.

The evaluation tool used post the completion of their learnings reflected the RN's high satisfaction with the ELNEC training as well as satisfaction with their job. During the learning seminars the RNs revealed real case scenarios that highlighted their development and competency in providing general palliative care.

\section{Implementing Telehealth in a Home Palliative Care} Service

WellMed Network Florida

Tampa, FL

Team/Contact Information:

Deidra R. Woods, MD

Palliative Medicine Physician

WellMed Network Florida

101 S. Hoover Boulevard

Tampa, FL 33609

(813) 261-3061

deidrawoodsduke@verizon.net

Project Team Members:

Thomas Hendershott

Deidra R. Woods, MD

Topic: Measuring impact and value

Description:

Telehealth is one of the fastest growing areas in the healthcare industry today. Virtual provider visits can extend a provider's capacity and geographic coverage, minimizing engagement in non-value added activities (e.g., driving). WellMed Network of Florida (a Medicare Advantage Plan) has recently implemented a Palliative Care Program that uses telehealth as one of its foundational technologies. To date, five palliative nurse case managers, two advance practice nurses and one physician have engaged in adopting this tool for use in our palliative service. This Poster Session will provide an in depth review of the implementation successes, challenges, and discoveries that we have encountered over the first six months of our program. We will outline the drivers and caveats of the technology, our staffing model, and our day-to-day processes and provide a description of our software/systems.

We will share our discoveries, expected and unexpected. In addition, lessons learned about users, both professional and lay users. Our current palliative care program is poised for extensive expansion, with plans for telehealth to be included as one tool to achieve growth. Prerequisite to that expansion is the establishment of a solid business, clinical and technical model, which we have achieved. Moreover, we are prepared to propagate this Telehealth program throughout the organization. 
76. Automatic Screening to Identify Palliative Care Patients Western Connecticut Health Network

Danbury, CT

Team/Contact Information:

Karen Mulvihill, DNP

Network Director Palliative Care

Western Connecticut Health Network

24 Hospital Avenue

Danbury, CT 06810

(203) 885-6267

karen.mulvihill@wchn.org

Project Team Members:

Damanjeet Chaubey, MD, MPH

Moreen Donahue, DNP, FAAN

Karen Mulvihill, DNP, AHCPN

Topic: Quality improvement

Description:

Staff from an established Palliative Care Team (PCT) in western Connecticut identified a gap in identifying patients appropriate for a palliative care consults. Patients were only being identified following multiple hospitalizations, despite having a serious, life-limiting illness. The PCT recognized that hospitalized patients could benefit from palliative care consult were being missed and not seen in a timely manner which was leading to increased readmission and longer length of stay. The Palliative Care Team (PCT) examined medical records of patient to determine to determine baseline data in time from admission to palliative care consults and their length of stay. Results showed the Team's median days from admission to initial consult was 3 days and the average length of stay was around 11 days. A palliative care screening tool was created and implemented as part of the admission nursing assessment. The screening was to be done with all admissions, and can also be repeated anytime during hospitalization. Education about the new process and tool was conducted for all physicians and nurses hospital wide. In Patients who scored an eight or higher, an automatic consult was placed into the medical record, and an alert was sent to the team. The palliative care team then measured the number of referrals, time to consult, length of stay, and readmission. Forty-one percent of patients admitted to the medical-surgical services have been screened. Since implementation of the screening tool, the palliative care service has seen and increases in consults, a decrease in days to consult to two, and a decrease in readmissions. Patient and family satisfaction has also increased as a result of early consult and creation of a patientcentered plan of care.

\section{Home-Based Palliative Care Reduces Hospital Readmissions}

Yuma Regional Medical Center

Yuma, AZ

Team/Contact Information:

Jeanne Elnadry, MD

Director of Palliative Care

Yuma Regional Medical Center

2400 S. Avenue A

Yuma, AZ 85364

(928) 336-2165

jelnadry@yumaregional.org

Project Team Members:

Jeanne Elnadry, $M D$

Susan Pancrazi, RN, BSN

Susan Lykins, RN, CCM, CPHM, IQCI
Topic: Measuring impact and value

Description:

Home visits have significant potential to address the complex issue of hospital readmissions within 30 days of prior discharge. Many patients who are readmitted to the hospital have advanced chronic illness and multiple chronic conditions. They often have poor social support networks and limitations of functional ability. Medication regimens are complex and often difficult to follow. Our palliative care intervention includes:

- Home visits by a palliative care physician

- Availability by cell phone

- Availability for emergency home visits during office hours

- Advance care planning/goals of care conversation

- Disease management education

- Development of plan for managing anticipated emergencies in the home

- Selected meds available in the home with instructions for how to use for specific emergencies

- Interdisciplinary team collaboration with Transitional Care Clinic, home health agencies, and home hospice care

This intervention is performed by a physician, with support of the transitional care clinic (which focuses on providing clinic-based care for the 30 days post hospital discharge). Intervention includes face-to-face encounters wherever the patient lives, a team approach by using home health agencies for nursing, social work, and rehabilitation therapies, or home hospice, telephone availability after office hours, availability home visits for emergencies that occurred during office hours.

Results: Data for initial 41 patients during the year prior to palliative care intervention compared to the year following palliative care intervention showed $67 \%$ reduction of hospital encounters (hospital admission, observation admission, or emergency department admission), from an average of 6.7 encounters per patient during the year prior to palliative care intervention, to 2.5 encounters during the subsequent year. Estimated direct cost per patient was $\$ 23,239$ during the year prior to intervention, and $\$ 8527$ during the subsequent year. During the year following initiation of the palliative care intervention, only 21 of the 41 patients had a hospital encounter.

Additional data for 30 patients shows a $71 \%$ reduction in hospital admissions, $75 \%$ reduction in readmissions within 30 days, and 69\% reduction in Emergency Department visits. Review of individual patient data shows that only 5 patients accounted for 89 encounters, the other 25 patients accounted for 77 encounters $017 \%$ of patients accounted for $54 \%$ of encounters.

Conclusions: A home-based palliative care intervention providing physician home visits significantly reduces hospital encounters and readmissions along with direct costs for patients with advanced illness.

\author{
78. Integrating Spiritual Care Into a Community Cancer \\ Center \\ Yuma Regional Cancer Center \\ Yuma, AZ \\ Team/Contact Information: \\ Laree Carlson, BSN, MA \\ Spiritual Care Resident \\ Yuma Regional Cancer Center \\ 1320 West 24th Street
}


Yuma, AZ 85364

(928) 328-9562

lcarlson@yumaregional.org

Project Team Members:

Laree Carlson, BSN, MA

Jeanne Elnadry, $M D$

Topic: Measuring impact and value

Description:

Background: Health care providers do not routinely screen for or address their patient's spiritual care needs. Yet a diagnosis of cancer, for many patients and families, is a profound challenge to their sense of well-being on multiple levels - physical, spiritual, emotional, social. Barriers to the provision of spiritual care in a community cancer center setting may include - limited time, scarcity of skilled spiritual care providers, poor understanding of the impact of spirituality on patient/family well-being, and concern for rigorous clinical attention to cancer treatment.

Methods: In September 2015, a chaplain resident from the Clinical Pastoral Education Residency Program joined the Supportive Care Clinic at Yuma Regional Cancer Center. The goal was to assess in an ongoing manner, the emotional and spiritual needs of complex patients, and to develop a plan to provide them with the opportunity to process their distress in real time. We began with the chaplain resident and palliative care physician seeing patients together during the weekly Supportive Care Clinic. The chaplain's presence with the physician helped to validate the value of the chaplain's intervention thereby opening new opportunities for dialogue in the context of each clinic visit. During this time, it was noted that the current single-item screening tool for spiritual distress did not identify those individuals at risk, and it was therefore not able to trigger referral for spiritual care.

Results: As patients and families developed a relationship with the chaplain, they began to request time with her. Through the practice of being present and presenting a supportive and nonjudgmental attitude, the chaplain was able to provide spiritual care to patients regardless of their belief system. Patients have reported improved quality of life as a result of the care provided through the chaplain/physician team. In addition, other supportive disciplines such as nurse navigation, case management, and physicians have sought out consultation and collaboration with the chaplain on an increasing basis.

Conclusions: Integration of spiritual care in a community cancer center has improved the overall perception of quality of care as patients and families begin to acquire tools that aid them in coping with the distresses accompanying their illness journey.

79. *A Primary Palliative Care Pathway for Hospital
Medicine
Center for Hospital Innovation and Improvement, Society of
Hospital Medicine and
The Hastings Center
Philadelphia, PA
Team/Contact Information:
Julia Ragland, MD, FHM
Center for Hospital Innovation and Improvement, Society of
Hospital Medicine and
The Hastings Center
1500 Spring Garden Street, Suite 501
Philadelphia, PA 19130
(617) 243-6573
jragland@partners.org

79. *A Primary Palliative Care Pathway for Hospital Center for Hospital Innovation and Improvement, Society of Hospital Medicine and

The Hastings Center

Philadelphia, PA

Team/Contact Information:

Center for Hospital Innovation and Improvement, Society of Hospital Medicine and

The Hastings Center

1500 Spring Garden Street, Suite 501

(617) 243-6573

jragland@partners.org
Project Team Members:

Julia Ragland, MD, FHM

Nancy Berlinger, PhD

Lynnea Mills, $M D$

Clareen Wiencek, RN, PhD, CNP, ACHPN

Brett Hendel-Paterson, $M D$

Jenna Goldstein, MA

Barbara Egan, MD, SFHM, FACP

Wendy Anderson, $M D, M S$

Topic: Integration of palliative care to specific settings (e.g., hospital, ICU, home, nursing home, clinic)

\section{Description:}

Background: Most Americans experience hospitalization in the last year of life. Unwanted medical interventions near the end of life, and their associated costs, have been a national concern for decades. Hospital palliative care consultation programs increase health care value by helping to ensure that patients do not receive unwanted treatments. However, the size of the palliative care specialist workforce relative to need creates obstacles to routine access for seriously ill patients. In recent years, palliative care specialists have articulated principles of "generalist" or "primary" palliative care reflecting core components that can be delivered by non-specialists.

Integrating Primary Palliative Care into Frontline Hospital Care

The frontline hospital clinician is a crucial partner in hospitalbased efforts to significantly expand the palliative care workforce and augment the consultation model, with the goal of meeting needs that routinely arise for seriously ill patients. Hospital medicine is the fastest-growing medical specialty in the United States. Nationwide, 44,000 hospitalist physicians, 80 percent of whom work in community hospitals, are responsible for the care of seriously ill hospitalized patients from admission through discharge, and for improving patient outcomes and health care utilization. Hospital medicine has developed award-winning models to translate safety and quality targets into clinical practice.

The Society of Hospital Medicine (SHM) has now joined forces with The Hastings Center, the source of landmark Guidelines on treatment decision-making and care for seriously ill people, to develop a model to integrate primary palliative care into normal hospital care. The SHM-Hastings Center team convened a national stakeholder meeting in July 2015 on "Improving End-of-Life Care in the Hospital," which produced a concept map of major themes, priorities, and challenges in translating proven processes of palliative care consultation into frontline clinical work.

Designing a Primary Palliative Care Pathway for Hospital Medicine: Result

An interdisciplinary Expert Panel of hospitalists, health care ethicists, and experts in acute-care nursing from the American Association of Critical-Care Nurses (AACN) collaborated to design a care pathway to support hospitalists initiating a key component of primary palliative care: discussing prognosis and goals of care and ensuring that patients' hospital and posthospital treatment and care are aligned with their preferences. The Expert Panel's work builds on research and insights from palliative care, patient safety, and quality improvement. This focused interdisciplinary effort to articulate and establish a new norm in hospital medicine aims to fill a pressing need for a practical, sustainable model to deliver high-value primary palliative care in the hospital setting.

Next Steps

The new pathway, practice standard, and implementation tools will be publicly available as a quality improvement 
Resource Room on SHM's web-based platform starting in Fall 2016, and will be shared through professional meetings, journals, and other dissemination venues. Future research will study implementation to create recommendations for model adaptation and refine evaluation metrics to support wider use.

\section{Acknowledgments}

This project was made possible by a major grant from the Milbank Foundation and was developed through an R3 grant from the Donaghue Foundation.

*Special Recognition Posters

80. *Evaluating Impact of a Statewide Medicaid Palliative Care Intervention

Community Care of North Carolina

Raleigh, NC

Team/Contact Information:

Jonathan Fischer, MD

Assistant Professor

Duke University Department of Community

and Family Medicine;

Duke Palliative Care; Community Care of NC

Community Care of North Carolina

2300 Rexwoods Drive, Suite 100

Raleigh, NC 27607

(919) 745-2350

jonathan.fischer@duke.edu

Project Team Members:

Jonathan Fischer, MD

Julian Thomas

Carlos Jackson

Topic: Measuring impact and value

Description:

Community Care of North Carolina (CCNC) is a communitybased, public-private partnership that takes a population management approach to improving health care and containing costs for North Carolina's most vulnerable populations. Through its 14 local network partners, CCNC creates over 1400 primary care "medical homes" in all 100 counties for Medicaid beneficiaries and uninsured people. For its palliative care (PC) initiative, CCNC has developed reliable systems to identify seriously ill Medicaid patients so that care managers (CMs) may offer opportunities for advance care planning, improved symptom management, enhanced psychosocial support and hospice referrals when appropriate. The current health care pathway unfortunately includes a trajectory of hospital admissions and high degrees of intervention for seriously ill patients. The PC initiative of CCNC looked to pause this high cost course, thereby allowing goals of care discussions and the possibility of greater concordance of patients' wishes with the care that they receive. The CCNC care managers' palliative care engagements may include assessments for physical symptoms, recognition of psychological stress, clarification of a patient's goals of care, completion of advance care planning documents, referrals to palliative care consultants or hospice, and coordination of care with the medical care team. The purpose of this evaluation was to determine if we could identify any "signal" that a PC intervention performed by CCNC care managers has impact on subsequent healthcare cost and utilization.

This evaluation examined healthcare costs and utilization for 836 deceased North Carolina Medicaid patients who met criteria for the evaluation: 207 receiving PC intervention, and 629 in the comparison group who did not. For the intervention group, the inpatient discharge around which the patient received the palliative care intervention was referred to as the index discharge. For the control group, the first inpatient visit that occurred during the last year of the person's life was identified as the index discharge. In order to further maximize comparability with the comparison group, we stratified patients according to when they died relative to the index discharge in both groups. Following the approach described above ensured that we were comparing patients in the intervention group with patients who had similar risk of mortality, risk of admission/ readmission, and similar time until mortality.

Four categories or strata of patients were created based on when death occurred relative to their earliest hospital admission during the year leading up to death. In nearly every strata, patients who received PC spent less, had fewer inpatient days, and had more hospice days during the months prior to death. When examining the cost impact overall across strata, patients in the intervention group spent $\$ 1,661$ less each month from the time they received the intervention until death. Patients receiving PC also had an average of 0.5 fewer inpatient days per month, and an increase of 0.7 hospice days per month.

Results from this evaluation provide compelling evidence that there is potential savings from palliative care interventions delivered by a care manager within a primary care setting.

*Special Recognition Posters

81. *Integrated Palliative Care in a Rural Healthcare System Harris Regional Hospital and Swain Community Hospital Sylva, NC

Team/Contact Information:

Morgan Allen, CHPCA

Administrative Director

Harris Regional Hospital and Swain Community Hospital

68 Hospital Road

Sylva, NC 28779

(828) 631-1702

morgan_allen@westcare.org

Topic: Health system strategies

Description:

Harris Regional Hospital is an 86-bed acute care hospital based in Sylva, NC which has a population of approximately 2,600. Swain Community Hospital is a 25-bed critical access hospital located 25-miles to the west of Sylva in Bryson City, NC with a population of 1,400 . These two hospitals, along with a network of physician practices, outpatient services, and post-acute programs make up a rural, integrated healthcare system serving western North Carolina. In 1995, Harris Regional Hospice was established to provide end-of-life care for the mountainous communities in the far western counties of North Carolina. In 2006, Harris Regional Hospital launched the first interdisciplinary team model of palliative care in the area. The program was re-launched in 2013 with a focus to connect palliative care to all aspects of the continuum of care in this rural environment. The current palliative care service in our rural five county regions includes:

- Inpatient service comprised of physicians, chaplain, social worker, and nurses providing care to nearly $35 \%$ of the inpatient volume on average.

- Community based service with a full interdisciplinary team providing comprehensive palliative care at home and in skilled nursing and assisted living facilities throughout five counties. 
- Outpatient medical clinic for chronic and advanced illness management, with a sub-focus on pediatric care and rare disorders such as Ehlers-Danlos syndrome.

- Comprehensive community advanced care planning providing education and consultation in completion of advanced directives.

The palliative care leadership at Harris and Swain have successfully proven the feasibility and sustainability of a comprehensive hospital and community based palliative care service amalgamated in a small, rural healthcare system. Strategic partnerships outside of the health system have also proven invaluable to the success of the program, including partnering with nearly a dozen skilled nursing and assisted living facilities. Internal partnerships with primary care providers and multiple specialists, hospital case management, therapy services, medical ethics, among others have been instrumental in the assimilation of the program across the continuum.

Harris and Swain have demonstrated the positive business impact credited to the palliative service, with significant revenue increases for the hospice program. Additional volume has also been noted for the home health service, in partnership with our home palliative care. These successes are a result of appropriate transitions of care within our community health system continuum, coordinated by the palliative care team. Quality of care outcomes attributed to the palliative care program include reduced hospital re-admissions, impact on patient satisfaction, and exemplary feedback from referring physicians. These achievements are all the product of an integrated palliative program with a rural hospital system, rural home health service and rural hospice all in one organization.

Future palliative care development for this rural system will include a multi-disciplinary clinic in partnership with Western Carolina University to provide medical, nursing, social services, nutritional counseling, physical therapy, and other comprehensive care services to patients with advanced illness and support educational opportunities for students in the health sciences disciplines.

*Special Recognition Posters

82. *CarePoint ${ }^{\mathrm{TM}}$ : A System Level Approach to CommunityBased Palliative Care

Interim Healthcare Home Care and Hospice

Lockhart, TX

Team/Contact Information:

Robert Parker

Interim Healthcare Home Care and Hospice

506 Brushy Branch Road

Lockhart, TX 78644

(806) 782-2976

bob.parker@interimhh.com

Project Team Members:

Robert Parker

Leeza Struwe

Lufei Young

Topic: Integration of palliative care to specific settings (e.g., hospital, ICU, home, nursing home, clinic)

Description:

Background: CBPC programs have potential to deliver improved quality of life \& cost-effective health outcomes for a vulnerable population. Research in the development \& evaluation of CBPC programs is scant. Lack of evidence has significant implications for a population in need of cost-effective care so- lutions that promote QoL, \& drive quality health outcomes at end of life. CarePoint ${ }^{\mathrm{TM}}$ is a CBPCP operated as a system of care by Falcon Healthcare, Inc., a franchisee of Interim Healthcare, reimbursed thru traditional fee-for-service payment mechanisms, accountable care organizations, and bundling agreements.

Objective: Evaluate CarePoint's effect on patient's selfreported symptom burden and QoL, advance care planning engagement, \& healthcare utilization; provider's impact on patient's care quality perception; \& system outcomes associated with aggregate costs.

Methods: 30-day pretest-posttest intervention. 56 patients who received services March - July 2016 were included. Participants were eligible if they resided in a home, skilled nursing facility, assisted living facility, or group home, at least 18-years of age, spoke English, and had Brief Mental Status Interview score 13+. Clinical data was collected thru prospective chart audits for ACP engagement, hospital, and ED utilization; Edmonton System Assessment System Revised scores for symptom burden, EuroQol EQ-5D-5L for health state, Patient Assessment of Care for Chronic Conditions for care quality agreement. Study included active CarePoint program locations - an average daily census of 647 patients covering two geographical regions within Texas. Two West Texas programs cover 41 counties, rural with an active daily census of 364 patients. 2 Central Texas programs cover 19 counties, slightly urban with an active daily census of 283 patients. Interdisciplinary team supports CarePoint, the intervention. Primary team includes certified hospice and palliative care advance practice nurses, registered nurses or licensed vocational nurses that provide direct medical/supportive care services under supervision of a board certified hospice and palliative care physician. Secondary team includes home health and hospice social workers, hospice chaplains, and volunteers.

Results: Fifty-two participants $(80.6 \%)$ completed the study. At 30-days hospitalizations were significantly reduced by $25 \%$ and ED utilization by $100 \%$ with an adjusted potential cost savings of $1.6 \mathrm{M}$ across the program. Patient's perceived care quality agreement improved significantly by $51.79 \%$. Reported symptom burden was statistically significantly different for pain $p=0.000$, depression $p=0.014$, anxiety $p=0.019$, and wellbeing $p=0.003$, and the overall EuroQol visual analog scale (EQ VAS) slightly improved from 47 to $51(0=$ worst health you can imagine; $100=$ best health you can imagine).

Conclusions: A CBPCP, delivered via medical and supportive care services, proved to be a feasible and effective delivery model in managing patients with end of life needs.

Implications: Pilot study adds to the scant body of evidence for CBPCP design \& evaluation as a foundation for other studies to compare outcomes, and determine long-term effects.

*Special Recognition Posters

83. *Growing the Tribe: Creation of a Regional Palliative Care Collaborative

Main Line Health Palliative Care

Wynnewood, PA

Team/Contact Information:

Christopher A. Jones, MD

Palliative Physician

Main Line Health Palliative Care

100 E. Lancaster Avenue, MOBS114

Wynnewood, PA 19072

(484) 476-2570

joneschr@mlhs.org 
Project Team Members:

Christopher Jones

Mary Pat Lynch

Susan B. Kristiniak

Amy Davis

Maria Doll-Shaw

Anessa Foxwell

Joshua Jones

Mary Kraemer

Brianna Morgan

Rupali Rajpathak

Arthur Siegal

Topic: Advocacy and coalition building

Description:

Breaking Bread and Building the Tribe: Creation of a Regional, Interdisciplinary Palliative Care Collaborative

\section{Objectives}

- Describe key initial steps when exploring the creation of a regional PC collaborative

- Explain potential short-term, intermediate, and aspirational goals for an interdisciplinary group of PC professionals

- Relate 5 lessons learned during the first year of the DVPCC

Palliative Care (PC) programs are flourishing nationally, with $90 \%$ of hospitals with 300 or more beds offering PC. Hospitals with 500 or more beds are expanding PC team sizes, averaging 7.8 full time equivalent positions in 2014. Despite this growth, smaller hospitals are less likely to have PC programs and those that do average less than half the staff members of larger institutions. With high burnout rates in PC (62\% in a 2014 survey with younger clinicians who feel isolated most susceptible); nurturing connections with colleagues may help sustain PC providers and programs long term.

Through this poster, we will outline for conference attendees a framework to build their own regional PC organizations by sharing the creation of the Delaware Valley Palliative Care Collaborative (DVPCC), a multidisciplinary group of PC clinicians from among the 58 hospitals and many hospices within 40 miles of Philadelphia. After reviewing our needs assessment, we will highlight the creation of our inter-institution planning committee and achievements of the first year (including 4 meetings and a membership that has grown to over 120 clinicians). Short-term successes crucial to maintaining momentum include a DVPCCbranded website with shared teaching files and presentation slide decks. The intermediate-term cultivation of a mentoring network will be highlighted and potential aspirational goals, including the benefits of an integrated PC course and securing 501(c)(3) nonprofit status, will be offered. Finally, we will devote significant space to sharing mistakes made and lessons learned, hoping to smooth the road for those in other geographic areas seeking to enhance and engage their region's PC tribe.

*Special Recognition Posters

84. *Early Palliative Care in Advanced Heart Failure North York General Hospital

Toronto, Ontario, Canada

Team/Contact Information: Karen Lock, NP

North York General Hospital

4001 Leslie Street
Toronto, Ontario M2K 1E1, Canada

(416) 756-6000 x4242

karen.lock@nygh.on.ca

Project Team Members:

Karen Lock

Wendy Cheung

Susan Woollard

Daphna Grossman

Nina Horvath

Dorothy Sullivan

Karen Weisz

Richard James

Collene Traille-Brown

Karen $\mathrm{Yu}$

Archna Patel

Shana Haberman

Janet Coldwell

Daryl Roitman

Warren Lewin

Topic: Integration of palliative care to specific settings (e.g., hospital, ICU, home, nursing home, clinic)

Description:

Heart Failure is a chronic, life-limiting illness that affects more than 600,000 Canadians and is the number one reason for hospitalization in Canada (Heart \& Stroke Foundation, 2016). With medical advances, this population continues to live longer. Prolonged survival is often correlated with increased symptom burden, compromised quality of life and increased care needs. Palliative care focuses on addressing and relieving symptoms of patients and families, and can be provided at any point during illness trajectory. However, most patients do not receive comprehensive palliative care until the last few days to weeks of life. It is believed that only 16-30\% of Canadians who could benefit from palliative care actually receive it (Office of the General Auditor of Ontario, 2014).

At North York General Hospital (NYGH), we believe "Our patients come first in everything that we do!'” In keeping with the philosophy, the Department of Cardiology collaborated with Palliative Care to establish an interprofessional Supportive Cardiology Program to address the palliative care needs of patients with advanced heart failure in July 2014. This program was funded as a 1-year pilot project through the Ministry of Health and Long-Term Care. Due to the unpredictable nature and varied progression of heart failure, the program objectives are to provide support for patients and families in early identification of goals of care, facilitate advance care planning, optimize symptom management, provide quality end-of-life care and connect patients/families to community resources including home-based palliative care with the hope to increase emergency room diversion, reduce recurrent hospitalizations, and increase patient/family satisfaction. This program serves both patients in the Heart Function Clinic and on the inpatient units.

As a result of the pilot project, the number of palliative care referrals for patients admitted to NYGH with heart failure increased in 2015 by $300 \%$ over a 2 -year period. For the patients served during the study period, palliative care consultation was associated with an increase in documented end-of-life conversations and advance care planning, as well as an increase in number of requests for "Do Not Resuscitate" code status. Palliative care integration has led to a practice change at our Heart Function Clinic and on the inpatient cardiology unit to include prognostic and future planning conversations earlier in the illness trajectory for patients with advanced heart failure.

The completion of the project has impacted on culture change and organizational commitment. The Supportive Car- 
diology Program has expanded to serve other incurable progressive chronic diseases. The palliative care team has established a formal collaboration with the respirologists and other interprofessional members to provide early palliative care to patients with advanced chronic obstructive pulmonary disease. Funding for a full-time nurse practitioner was secured and the clinic has been renamed to "Supportive Care Clinic." The NYGH team will share their experience with program development, use of referral criteria, model of care, screening tools, successes and challenges.

*Special Recognition Posters

85. *POLST, A Community Grassroots Initiative Northern Arizona Healthcare and Northern Arizona University Flagstaff, AZ

Team/Contact Information:

Mary Anne Hales Reynolds, PhD

Associate Professor

Northern Arizona Healthcare and Northern Arizona University 1200 N. Beaver Street

Flagstaff, AZ 86001

(928) 523-8209

mary-anne.reynolds@nau.edu

Project Team Members:

Mary Anne Reynolds, PhD, RN, ACNS-BC

Emmalee Kennedy, MD

Cindy Beckett, PhD, RNC-OB, LCCE

Dawn Rivas, RN, MS

Jennifer Guerrero, RN, BSN

Topic: Palliative care education

Description:

Provider Orders for Life-Sustaining Treatment (POLST) is a process that translates a patient's goals of care at the end of life into medical orders that follow the patient across healthcare settings. This signed document by the patient (or surrogate) and healthcare provider addresses the wishes of the patient with serious life-limiting illness. POLST support historically has been garnered through three pathways: grassroots efforts, regulatory changes, and legislation.

Arizona has unique barriers to POLST implementation given current legislation regarding prehospital DNR. While Arizona has been cognizant and active in all three areas, the state POLST task force has chosen to focus the majority of its efforts through the grassroots pathway, starting with implementing pilot programs throughout the state, documenting safety and efficacy of the program. The POLST Task Force and Thoughtful Life Conversations provide important community support from the state level. Both organizations are supported by the Arizona Hospital and Healthcare Association and work as liaisons with the national POLST organization, healthcare systems, payer groups, community action groups, EMS, and legislators.

The first successful pilot program was started in Flagstaff, Arizona at Flagstaff Medical Center (FMC). FMC is part of Northern Arizona Healthcare (NAH) a healthcare organization that covers over 50,000 square miles in Northern Arizona and provides comprehensive services to a largely rural and diverse population. The initial program was started under the guidance and support of the Palliative Care Department at FMC. Congestive heart failure patients with Stage D or C with severe comorbidities were offered participation in a POLST conversation related to goals of care and culminating in POLST form completion. A palliative care nurse assisted in communicating the form to the patient's health care team. Patients were followed for one year with intermittent follow up assessments for form changes, contact with the health care system (including prehospital), and patient/family perception of the usefulness of the form. The majority of patients approached accepted participation, although in follow up, many difficulties with communication of the POLST to other providers was noted.

This pilot study was then expanded by collaborating with Northern Arizona University School of Nursing (NAU). NAU is a public university also located in Flagstaff. NAH and NAU applied for and received grant money through a program sponsored by a Translational Health Research Initiative (THRIVE). THRIVE is a formal partnership between NAU and NAH created to support innovative population health research to improve health outcomes for all of the communities across Northern Arizona. With the grant, the research efforts are currently expanding to include a larger and more diverse hospitalized patient population. In addition to introducing more patients and their families to the POLST conversation, the grant is also supporting POLST education and awareness programs directed to community health care providers. Northern Arizona is seeing community wide benefits of multiple groups working together to advocate for patient choice in care during a serious illness.

*Special Recognition Posters

86. *Collaborative Care across the Silos: Respecting Wishes and Decreasing Unwanted Readmissions

Northwell Health

Long Island Jewish Medical Center

New Hyde Park, NY

Team/Contact Information:

Tara Liberman, DO

Associate Chief, Northwell health

Northwell Health

Long Island Jewish Medical Center

270-05 76th Avenue

New Hyde Park, NY 11040

(718) 470-3115

TLiberma@northwell.edu

Project Team Members:

Tara Liberman, DO

Adriana Calosso, NP

Mary Curtis, PhD

Regina Roofeh, $B S$

Maria Carney, $M D$

Topic: Care transitions

Description:

Background: According to the Dartmouth Atlas, the Long Island region sees higher rates of hospital utilization in the last 6 months of life and higher rates of in-hospital death in this population as compared to other regions of the country. Nationally, of those who indicated a preference to die at home, $55 \%$ died in the hospital. The aim of this project is to create a collaborative among a tertiary care hospital, a nearby nursing home facility and a community agency in an effort to deliver geriatric palliative care across all settings, while reducing unwanted hospital re-admissions and inpatient deaths. The hypothesis is that palliative care coordination will lead to fewer hospital readmissions and fewer deaths in the hospital in a patient population known to have multiple readmissions. The goal is to provide a palliative consult on individuals with advanced illness and a history of multiple readmissions.

Methods: The Geriatric and Palliative (GAP) care team at Long Island Jewish Medical Center (LIJ) consulted patients 
with advanced illness arriving from Parker Jewish Institute for Healthcare \& Rehabilitation, a skilled nursing facility, who had been hospitalized within the preceding 3 months. Upon discharge from LIJ to Parker Jewish Institute, a nurse practitioner from the GAP consult service provided a telephone call to the designated staff member at the nursing facility. Transition of care included discussions regarding diagnosis, health status, goals of care, and established advance directives. Data collected included: post-intervention readmissions, location and date of discharge, additional services provided (e.g., hospice and home care), and final disposition. A community agency supported patients and families for their psychosocial needs.

Results: Two hundred patients have been included in the study to date, with a median age of 82 years; 89 males, 111 females. Nearly all patients $(98.7 \%)$ have documented advance directives, with 55\% wanting "Do Not Resuscitate" orders and $44.5 \%$ wanting "Full code" status. Of included patients, 174 were discharged back into the community. While $97.5 \%$ of patients had one or more hospitalizations within 3 months prior to the intervention, only $47.7 \%$ of the discharged patients were hospitalized within 3 months post-intervention, a $49.8 \%$ decline. Of the discharged patients, 99 have died to date, with $62.6 \%$ dying outside of the hospital in the nursing facility, home, or hospice, and $37.4 \%$ dying in the hospital setting consistent with their goals of care.

Conclusion: As our population ages, conversations regarding advanced directives, goals of care and prognosis, proper documentation, and communication to other facilities can decrease unwanted readmissions. While the overwhelming majority of patients (together with their families and caregivers) have been comfortable with documenting their advance directives, there are still a significant number that want full code status and further re-hospitalization and utilization of hospital resources. As the project progresses, findings such as this shed light on the many factors that may lead to in-hospital deaths, as there are clearly both systemic and human factors involved in each patient's trajectory through the care continuum.

*Special Recognition Posters

87. *New Model: Integrating Palliative Across Care Settings Silvercrest Center for Nursing and Rehabilitation

New York Presbyterian/Queens

Briarwood, NY

Team/Contact Information:

Marta Kazandjian, MA CCC SLP BCS-S

Senior Director, Speech Pathology, Clinical Initiatives and Patient Experience; Clinical Director Speech Pathology

Silvercrest Center for Nursing and Rehabilitation; New York Presbyterian/ Queens

144 - 45 87th Avenue

Briarwood, $N Y 11435$

(718) 670-2736

mkazandjian@silvercrest.org

Project Team Members:

Marta Kazandjian, MA, CCC, SLP, BCS-S

Fernando Kawaii, MD, FACP

Maureen Peters, ACSW, LCSW, ACHP-SW

John Zavorinos, $M D$

Michael Gale, MD

Cynthia X. Pan, MD, FACP

Topic: Integration of palliative care to specific settings (e.g., hospital, ICU, home, nursing home, clinic)
Description: Seriously ill patients with complex medical problems undergo many transitions across hospital and nursing homes (NH). During these transitions, patients experience reduced continuity of care and important goals of care information may not be transmitted. We used a hospital based Hospice \& Palliative Medicine (HPM) fellowship training model to link the interdisciplinary team (IDT) in the $\mathrm{NH}$ to the palliative care IDT at the hospital. ACGME mandates NH rotation for HPM palliative care fellows. A typical rotation in a $\mathrm{NH}$ would be brief, e.g., one month. We chose a longer experience (weekly, half-day, rotation over a year) to provide continuity for both patients and physicians.

Objectives:

1) To illustrate a model for integrated palliative care services across $\mathrm{NH}$ and hospital.

2) To demonstrate a unique model in fellowship rotations to foster IDT relationships between hospitals, fellowship programs and NHs.

3) To measure number of hospitalizations among $\mathrm{NH}$ patients before and after IDT palliative care consults.

4) To measure the number of advance directives completed in $\mathrm{NH}$ patients before and after palliative care consults.

Methods: Prospective study of seriously ill patients who were referred for palliative care consults, at Silvercrest Center for Nursing and Rehabilitation (SCNR), a 320 bed post- acute care facility that contains 90 plus vent/pulmonary beds, with many chronically, critically ill patients at high risk for recurrent admissions. SCNR is closely affiliated with New York Presbyterian Queens (NYPQ), where the fellowship is based. Each week, physicians (palliative care attending and fellow) along with NH IDT members (social worker, speech pathologist, nurse practitioner, dietician, nursing assistant and integrative health coach) provided palliative care consults at the request of primary care physicians. Consultants provided pain and symptom assessment and management, goals of care reviews, family meetings, and options for withdrawing life support measures if consistent with patient/family goals. The yearlong exposure allowed fellows to continue goals of care discussions as patients were transferred between $\mathrm{NH}$ and hospital, as their disease progressed. This allowed conversations to continue when initiated at either site when patient/families were not ready to make decisions.

Results: Between Aug 2015 and June 2016, the consult team saw 61 patients, and provided total of 115 visits. There was a reduction of total \# of hospitalizations before and after palliative care consultation (Pre $=43$, and post $=18$ ). There was an increase in total \# Advance Directives (DNR, DNH, DNI) after Consults (pre $=74$; post $=92$ ). There were 5 cases where families elected to withdraw life sustaining treatments (vent support and/ or tube feeding). There was culture change with both acute and long term care team members, with increased understanding of palliative care principles, and NH staff appreciating the value of supporting the patient's and family's goals of care.

Conclusion: The increasing recognition of the role of palliative care provided many opportunities to improve patient experience and family satisfaction. This HPM fellowship training model provided enhanced continuity of care and IDT collaboration between hospital and NH. This model appeared to reduce avoidable hospitalizations and increase advance directives completion.

\footnotetext{
*Special Recognition Posters
} 
88. *A Visual Guide of a Palliative Care Workflow in a Hospital Setting

Trinitas Regional Medical Center

Elizabeth, NJ

Team/Contact Information:

Geraldine Cruz, MSN, RN, CHPN, CCRN

Director of Palliative Care

Trinitas Regional Medical Center

225 Williamson Street

Elizabeth, NJ 07202

(908) 994-5851

gcruz@trinitas.org

Project Team Members:

Geraldine Cruz, MSN, RN, CHPN, CCRN

Diane Reehil, MSN, RN, GNP, CNN

Carmen Mercado, $M S, R N, A P N-C$

Clare Cruz, MSN, RN, BC

Topic: Integration of palliative care to specific settings (e.g., hospital, ICU, home, nursing home, clinic)

Description:

Trinitas Regional Medical Center (TRMC) received the initial Joint Commission Certification in Advanced Palliative Care in 2013 and redesignation in 2015. The initial Joint Commission Certification application process provided us the opportunity to challenge and reexamine Palliative Care (PC) workflow processes in the hospital. The result is a development of a workflow diagram that provided us the big picture of the continuum of Palliative Care program at TRMC. This process mapping displays the integration and interdisciplinary collaboration that occurs within the organization. A Palliative Care consult is triggered from five different sources; nursing admission assessment, physician's order, multidisciplinary discharge rounds, ancillary request, and family request. The consult reaches the PC nurse either verbally or via the electronic health record (EHR). PC consult order entered in the EHR sends an email notification to the PC team, and automatically populates the patient in the PC list. PC team activities follows, which includes, initial assessment, family meeting, weekly Palliative multidisciplinary rounds (Director of PC, Social Worker, Pastoral Care, PC Liaison Physician, ICU Intensivist, Advance Nurse Practitioners), and follow up visits. This visual diagram serves as an educational tool and a structure for the organization to follow.

*Special Recognition Posters

89. *Technological Strategies To Improve the Timeliness and Efficiency Palliative Care Consults

Trinitas Regional Medical Center

Elizabeth, NJ

Team/Contact Information:

Geraldine Cruz, MSN, RN, CHPN, CCRN

Director of Palliative Care

Trinitas Regional Medical Center

225 Williamson Street

Elizabeth, NJ 07202

(908) 994-5851

gcruz@trinitas.org

Project Team Members:

Geraldine Cruz, MSN, RN, CHPN, CCRN

Hana Baudendistel, $M S, B S N, R N, N E-B C$

Clare Cruz, MSN, BSN, RN, BC

Valerie Ramsberger, MSN, RN, ACHPN

Topic: Leveraging technology (e.g., EMR, telehealth)

\section{Description:}

The purpose of this project is to identify and implement technological strategies to improve the timeliness and efficiency of consults to the Palliative Care (PC) team so they may initiate the physical, psychosocial and spiritual needs assessment process.

Trinitas Regional Medical Center (TRMC) received The Joint Commission (TJC) Certification in Advanced Palliative Care in 2013 and redesignation in 2015. As a TJC certified, we are held to a set of standards and measures that guide our program. We set our target goal for initiation of the Palliative care initial assessment at $90 \%$. In 2013, our measure of success for this goal was below the $90 \%$ threshold. We were challenged to explore technology to assist us in reaching our goal. Patients referred to the PC program must be assessed within 24 hours by the PC RN and within 48 hours by the Social Worker and Pastoral Care Staff.

Our objectives include;

(1) To provide immediate notification of a consult to PC staff

(2) To prioritize and respond to referrals in a timely manner

(3) To improve timeliness of interdisciplinary assessment, comprehensive care and management of physical, psychological, emotional and spiritual needs of patients and families with chronic debilitating or lifethreatening illness

A variety of technological methods were utilized to improve our measure of success above the $90 \%$ threshold.

Technological Strategies:

(1) PC triggers embedded in the electronic Nursing Admission Assessment

(2) PC Consult order in EHR

(3) Real Time List of patients with PC consult order

(4) Email Notification

(5) Utilization of Mobile Phone

(6) Remote access to EHR.

These strategies resulted in:

(a) a $15 \%$ increase in Total Volume of PC Consults from 2013-2014

(b) Timeliness of Initial Assessment by PC-RN within 24 hours, increased compliance from $87 \%$ to $100 \%$,

(c) Timeliness of Psychosocial Needs Assessment within 48 hours, increased compliance from $71 \%$ to $95 \%$,

(d) Timeliness of Spiritual Needs Assessment within 48 hours, increased compliance from $48 \%$ to $98 \%$.

In conclusion, we learned that technology was the catalyst in expediting PC consults to our team as demonstrated by exceeding our $90 \%$ goal for initiation of PC consults. This led to a progressive increase in the number of PC consults. We also learned that during off-hours, holidays and weekends, the covering off-site PC staff has no EHR access to complete the initial assessment. In 2014, a laptop with secured, remote access was provided for the covering PC RN or Physician as a solution to this barrier. Implications for future use include leveraging technology to continue to improve other aspects of our PC program as well as other care services at TRMC.

\footnotetext{
*Special Recognition Posters
} 
90. *UCLA Health Advance Care Planning Initiative

University of California, Los Angeles

Los Angeles, CA

Team/Contact Information:

Anne M. Walling, MD

Assistant Professor in Residence

University of California, Los Angeles

911 Broxton Avenue

Los Angeles, CA 90024

(310) 794-2284

awalling@mednet.ucla.edu

Project Team Members:

Anne M. Walling

Jennie Kung

Frances Watts

Judy Thomas

Shirley Otis-Green

Neil Wenger

Topic: Health system strategies

Description:

Objectives of Program/Intervention: The UCLA Health System, in concert with the Coalition for Compassionate Care of California, built on institutional support to develop an advance care planning (ACP) model to maximize the health outcomes for patients including optimal care at the end of life. The objectives of the program are:

1. Develop a model that stratifies patients into groups based on clinical characteristics and readiness for ACP and focuses ACP on consideration of goals for care and values concerning future health states. For patients with advanced disease, this model cultivates prognostic awareness in anticipation of consideration of future health states.

2. Develop an infrastructure and training program so that faculty and staff expect ACP to be performed, Communication Promoters embedded at critical locations prompt specialized ACP with advanced illness patients, and special efforts target vulnerable aspects of care continuity such as transitions.

3. Incorporate measurement of processes and outcomes of ACP including targeted denominator definitions and collection of data reflecting the experience of care at the end of life.

Description of Program/Intervention: The UCLA Health ACP initiative is creating a system-wide mechanism to routinely engage patients in considerations about future health care and to elicit patient preferences. The ACP process aims to be reproducible across clinicians so that the iterative process of information sharing and decision making can occur over time and across venues of care and between providers. In addition, the mechanism is designed to integrate seamlessly with the UCLA Epic-based electronic health record so that patient preferences are elicited, preserved and iteratively updated in a fashion that will inform all providers and ensure that care decisions incorporate in real time patient's goals and preferences.

The initiative, guided by a multidisciplinary team of stakeholders, disseminates ACP across the health system by implementing the following components:

(1) A standardized ACP process

(2) Materials to inform patients and promote ACP.
(3) Training mechanism that produces individuals who can reproducibly implement the standardized ACP process in a clinically astute fashion.

(4) CareConnect infrastructure for maintaining ACP preferences including novel Goals of care notes that can be iteratively updated and applied at the point of care

(5) Metrics to evaluate the ACP activities and drive quality improvement - These include process measures such as advance directives and Goals of care notes, and outcome measures such as venue of death, utilization at the end of life, and implementation of the bereaved family survey.

Measures of Success and Findings to Date: Changes in advance care planning have been seen for the health system as a whole: Use of Goals of care notes for patients with advanced illness has increased from less than $1 \%$ to more than $10 \%$. Hospice use among decedents has increased from 5\% to $20 \%$. ICU use and ICU length of stay has decreased by $20 \%$ among patients with advanced cancer who died.

*Special Recognition Posters

\section{1. *How Standardized Data Collection Informs Palliative Care}

Palliative Care Program, University of California, San Francisco San Francisco, CA

Team/Contact Information:

David L. O'Riordan, PhD

Senior Research Specialist

Palliative Care Program, University of California,

San Francisco

533 Parnassus Avenue

San Francisco, CA 94143

(415) 476-6792

doriordan@medicine.ucsf.edu

Project Team Members:

Kara Bischoff, $M D$

Angela Marks, MSEd

David L. O'Riordan, PhD

Steve Z. Pantilat, $M D$

Topic: Measuring impact and value

Description:

Introduction: The Palliative Care Quality Network (PCQN) is a large consortium of palliative care (PC) teams in the United States who are collecting standardized data about the practice of PC.

Objective: Describe patients referred to inpatient PC, care provided by PC teams, and clinical outcomes.

Methods: Seventy-one PCQN members entered data on 48,290 patient encounters between January 3, 2012 and June 30, 2016.

Results: Patients referred to PC were an average of 72 years old (median $=74)$ and were significantly debilitated (mean palliative performance scale $=35.2$ ). The most common diagnosis leading to PC consult was cancer (32.8\%). Common reasons for referral to PC were goals of care discussions $(73.1 \%)$, followed by pain management $(20.2 \%)$, and providing support for patient/family (20.0\%). Patients were referred to PC an average of 4.7 days $($ median $=2)$ after hospital admission and were followed by the PC team for an average of 5.0 days (median $=3.0)$. Disciplines involved in PC consultations included physician (50.5\%), social worker $(36.5 \%)$, registered nurse $(33.4 \%)$, and chaplain $(31.8 \%)$. 
Of patients with moderate to severe symptoms, $68.5 \%$ reported improved pain from the 1 st to 2 nd PC assessment, $78.4 \%$ reported improved nausea, $65.9 \%$ reported improved dyspnea, and $65.7 \%$ reported improved anxiety.

A surrogate decision maker was identified for $95.2 \%$ of patients seen by PC. On average, patients had 1.2 family meetings with the PC team (median=1). Forty-four percent of patients had their code status clarified, $12.2 \%$ had a POLST form completed, and $3.1 \%$ completed an advanced directive. Three quarters of patients were discharged alive, with home being the most common discharge location (48.2\%). Hospice services were arranged for $38.0 \%$ of patients.

Conclusion: This multicenter dataset allows us to understand the services provided by inpatient PC teams, and the impact of that work. These data can be used to define best practices and drive quality improvement in PC.

*Special Recognition Posters

92. *A Medicare Advantage Clinic CHF/Palliative Care
Program
WellMed Medical Management
San Antonio, TX
Team/Contact Information:
Elizabeth Glazier, MD
Director, Palliative Care
WellMed Medical Management
8657 Fredericksburg Road
San Antonio, TX 78240
(888) 781-9355
eglazier@wellmed.net
Project Team Members:
Elizabeth J. Glazier, MD
Lilliana Oakes
Rosa Vizcarra

Linda May

Ruben Mata, NP

Michael Almaleh, MD

Topic: Integration of palliative care to specific settings (e.g., hospital, ICU, home, nursing home, clinic)

\section{Description:}

Headquartered in San Antonio, Texas, WellMed is a healthcare delivery network serving more than 227,000 patients, mostly Medicare-eligible seniors, in Texas and Florida. The WellMed Palliative Care Program encompasses the entire spectrum of patient care; engaging patients in the inpatient arena, skilled nursing facility, subspecialty clinic and in the comfort of their own home. As a non-academic, community practice, building a multi-disciplinary, subspecialty clinic involving palliative care with private cardiologists was a unique endeavor.

In November 2015, we expanded from providing subspecialty palliative care in a patient's primary care clinic to the creation of a multidisciplinary palliative care clinic in collaboration with cardiology for our CHF patients. Each patient is seen by a cardiologist and a palliative care-trained physician during each clinic visit. Most of the patients are NYHA Class III and IV. All patients receive a full geriatric assessment to include cognitive, emotional, social, functional, and medical assessments. We will share our experience over the last year in ensuring that advance care planning is introduced and documented in the EMR for this very high risk subset of patients. We were able to improve completion of advance care documentation for these patients from no advance care planning to $85 \%$. We will also share our successes in improving symptom management, primarily dyspnea, fatigue, depression and delaying functional decline.

\footnotetext{
*Special Recognition Posters
} 